\title{
Max Weber
}

Économiste et sociologue allemand [1864-1920]

(1919)

\section{LE SAVANT \\ ET LE POLITIQUE}

Un document produit en version numérique par Jean-Marie Tremblay, bénévole, Professeur associé, Université du Québec à Chicoutimi

Page web. Courriel: jean-marie tremblay@uqac.ca

Site web pédagogique : $\underline{\text { http://jmt-sociologue.uqac.ca/ }}$

Dans le cadre de: "Les classiques des sciences sociales" Une bibliothèque numérique fondée et dirigée par Jean-Marie Tremblay, professeur de sociologie au Cégep de Chicoutimi Site web: http://classiques.uqac.ca/

Une collection développée en collaboration avec la Bibliothèque Paul-Émile-Boulet de l'Université du Québec à Chicoutimi

Site web: http://bibliotheque.uqac.ca/ 


\section{Politique d'utilisation de la bibliothèque des Classiques}

Toute reproduction et rediffusion de nos fichiers est interdite, même avec la mention de leur provenance, sans l'autorisation formelle, écrite, du fondateur des Classiques des sciences sociales, Jean-Marie Tremblay, sociologue.

Les fichiers des Classiques des sciences sociales ne peuvent sans autorisation formelle:

- être hébergés (en fichier ou page web, en totalité ou en partie) sur un serveur autre que celui des Classiques.

- servir de base de travail à un autre fichier modifié ensuite par tout autre moyen (couleur, police, mise en page, extraits, support, etc...),

Les fichiers (.html, .doc, .pdf, .rtf, .jpg, .gif) disponibles sur le site Les Classiques des sciences sociales sont la propriété des Classiques des sciences sociales, un organisme à but non lucratif composé exclusivement de bénévoles.

Ils sont disponibles pour une utilisation intellectuelle et personnelle et, en aucun cas, commerciale. Toute utilisation à des fins commerciales des fichiers sur ce site est strictement interdite et toute rediffusion est également strictement interdite.

\section{L'accès à notre travail est libre et gratuit à tous les utilisa- teurs. C'est notre mission.}

Jean-Marie Tremblay, sociologue

Fondateur et Président-directeur général, LES CLASSIQUES DES SCIENCES SOCIALES. 
Cette édition électronique a été réalisée par Jean-Marie Tremblay, professeur associé, Université du Québec à Chicoutimi, à partir du livre de :

\section{Max Weber}

\section{Le savant et le politique (1919)}

Paris : Union Générale d'Éditions, 1963, 186 pages. Collection : Le Monde en 10-18.

Polices de caractères utilisées :

Pour le texte : Times New Roman, 14 points.

Pour les citations et les notes de bas de page : Times New Roman 12 points.

Édition électronique réalisée avec le traitement de textes Microsoft Word 2008 pour Macintosh le 4 octobre 2014, révisé le 4 avril 2018.

Mise en page sur papier format : LETTRE US, 8.5 " x 11 ". 


\section{Table des matières}

INTRODUCTION par Raymond Aron [5]

LE MÉTIER ET LA VOCATION DE SAVANT [53]

LE MÉTIER ET LA VOCATION D’HOMME POLITIQUE [99] 
[5]

\title{
INTRODUCTION
}

\author{
Par \\ Raymond ARON
}

$\underline{\text { Retour à la table des matières }}$

[6] 
Max Weber a été un homme de science, il n'a été ni un homme politique ni un homme d'État, occasionnellement journaliste politique. Mais il a été, toute sa vie, passionnément soucieux de la chose publique, il n'a cessé d'éprouver une sorte de nostalgie de la politique, comme si la fin ultime de sa pensée aurait dû être la participation à l'action.

Il appartenait à la génération qui assista, arrivant à l'âge d'homme, à l'épanouissement de l'empire allemand, à la chute de Bismarck et à la prise des responsabilités par le jeune empereur. Les quinze dernières années du $\mathrm{XIX}^{\mathrm{e}}$ siècle, pour Max Weber les années de formation entre vingt et trente-cinq ans, sont marquées à la fois par le développement de la législation sociale, par les premières interventions personnelles de l'empereur dans la diplomatie, plus profondément encore par la réflexion sur l'héritage bismarckien. Quelle est la mission de l'Allemagne, après l'achèvement de l'unité ? Quel doit être son rôle sur la scène mondiale ? Quel régime est susceptible de rétablir l'unité de la nation ? La génération de Max Weber se pose spontanément de telles questions auxquelles l'histoire devait donner de tragiques réponses.

Des motifs plus personnels expliquent aussi son attitude. Il n'a cessé de souligner que la politique n'avait rien à faire dans les salles de cours, il a répété [8] que les vertus du politique étaient incompatibles avec celles du savant. Mais son souci de séparer n'était pas plus aigu que sa conscience du lien entre les deux activités. On ne peut pas être en même temps homme d'action et homme d'études, sans porter atteinte à la dignité de l'un et de l'autre métier, sans manquer à la vocation et de l'un et de l'autre. Mais on peut prendre des positions politiques en dehors de l'université, et la possession du savoir objectif, si 
elle n'est peut-être pas indispensable, est à coup sûr favorable à une action raisonnable. En bref, les relations entre science et politique, dans la pensée de Max Weber, ne sont pas caractérisées seulement, comme on le dit toujours, par la distinction nécessaire. La science qu'il conçoit est celle qui est susceptible de servir l'homme d'action, de même que l'attitude de celui-ci diffère en sa fin, mais non pas en sa structure, de celle de l'homme de science.

L'homme d'action est celui qui, en une conjoncture singulière et unique, choisit en fonction de ses valeurs et introduit dans le réseau du déterminisme un fait nouveau. Les conséquences de la décision prise ne sont pas rigoureusement prévisibles, dans la mesure même où la conjoncture est unique. Il n'y a de prévision scientifique que des successions d'événements susceptibles de se répéter, en d'autres termes de rapports dégagés du concret et élevés à un certain niveau de généralité. La décision raisonnable n'en exige pas moins que l'on applique à la conjoncture l'ensemble des connaissances abstraites dont on dispose, non pour éliminer, mais pour réduire et pour isoler l'élément d'imprévisible singularité. Une science qui analyse les rapports de cause à effet, telle que la souhaitait Max Weber en théorie, est donc celle même qui répond aux besoins de l'homme d'action.

La théorie de la causalité historique, fondée sur des calculs rétrospectifs de probabilité - qu'est-ce qui se serait passé si... ? - n'est rien de plus que la reconstitution approximative des délibérations qui ont été ou qui auraient pu être celles des acteurs.

Agir raisonnablement, c'est, après réflexion, prendre la décision qui donne la meilleure chance d'atteindre le but que l'on vise. Une théorie de l'action est une théorie du risque en même temps qu'une théorie de la causalité. L'historien qui s'interroge [9] sur la causalité historique ranime, après coup, dans son esprit, les possibles évanouis que les acteurs envisageaient ou auraient pu envisager dans les délibérations qui précédèrent leur décision.

L'interrogation causale ne s'applique pas seulement à des décisions réfléchies d'un ou de plusieurs individus. On pose la question : que se serait-il passé si... ? aussi bien à propos d'événements qui n'ont été voulus par personne (intervention de phénomènes physiques, tempêtes, épuisement des mines d'or, défaite ou victoire dans une bataille, etc.) qu'à propos de décisions personnelles. L'effort pour éviter l'illu- 
sion rétrospective de fatalité n'en est pas moins caractéristique de l'historien politique, de l'historien qui, s'intéressant aux hommes et à leurs luttes, veut sauvegarder, dans la résurrection du passé, la dimension propre de l'action - à savoir l'incertitude de l'avenir, incertitude qui ne saurait être sauvegardée par l'historien qu'en maintenant, contre la sanction de l'événement, que le réel n'était pas à l'avance écrit et qu'il dépendait de telles personnes ou de telles circonstances que la marche de l'histoire fût autre.

Le lien entre la science et la politique de Max Weber apparaît tout aussi étroit si l'on considère l'autre aspect, non plus la relation causale mais les valeurs, rapport aux valeurs dans le cas de la science, affirmation de valeurs dans celui de l'action. Le choix des faits, l'élaboration des concepts, la détermination de l'objet, disait Max Weber, sont marqués par l'orientation de notre curiosité. La science naturelle sélectionne, dans l'infini du donné sensible, les phénomènes susceptibles de se répéter et construit l'édifice des lois. La science de la « culture » sélectionne, dans l'infini des événements humains, ce qui se rapporte aux valeurs, valeurs des contemporains ou valeurs de l'historien, et élabore soit l'histoire, si le savant fixe son attention sur la suite unique des faits on des sociétés, soit les diverses sciences sociales qui considèrent les consécutions régulières ou les ensembles relativement stables.

La science historique ou la science de "culture », telle que la concevait Max Weber, était la compréhension de la manière dont les hommes avaient vécu, du sens qu'ils avaient donné à leur existence, [10] de la hiérarchie qu'ils avaient établie entre les valeurs, cependant que l'action politique est l'effort, dans des circonstances que nous n'avons pas choisies, pour promouvoir ces valeurs, constitutives de notre communauté et de notre être même.

La compréhension d'autrui n'implique pas la réflexion sur soimême. La compréhension de l'action menée par les autres dans le passé ne conduit pas nécessairement à la volonté d'agir dans le présent. Il n'y en a pas moins, philosophiquement, et, pour employer le jargon à la mode, existentiellement, un lien entre la connaissance de soi et celle des autres, entre la résurrection des luttes que se sont livrées les hommes disparus et la prise actuelle de position. 
En fait, il ne manque pas d'historiens qui s'efforcent de comprendre les existences vécues par les autres sans s'interroger sur celle qu'ils vivent eux-mêmes. Il ne manque pas de politiques qui ne mettent pas en relation leur métier avec le sens ultime qu'eux-mêmes ou la collectivité donnent aujourd'hui à leur existence. En droit, l'exploration du passé ne se sépare pas de la prise de conscience de soi-même, en droit l'action n'est humaine qu'à la condition de se situer elle-même dans le cours des événements et par référence aux objectifs suprêmes. La réciprocité entre la rencontre avec l'autre et la découverte de soi est donnée dans l'activité même de l'historien. La réciprocité entre la connaissance et l'action est immanente à l'existence non de l'historien, mais de l'homme historique. Max Weber interdisait au professeur de prendre parti dans les querelles du Forum, à l'intérieur de l'Université, mais il ne pouvait pas ne pas considérer l'action, au moins par la parole ou par la plume, comme l'aboutissement de son travail.

On s'est demandé dans quelle mesure la pensée propre de Max Weber s'exprime adéquatement dans le vocabulaire et les catégories du néo-kantisme de Rickert. La phénoménologie de Husserl, qu'il a connue mais peu utilisée, lui aurait, me semble-t-il, fourni l'outil philosophique et logique qu'il cherchait. Elle lui aurait évité, dans ses études sur la compréhension, l'oscillation entre le "psychologisme " de Jaspers (à l'époque où celui-ci écrivait sa psycho-pathologie) et la voie détournée du néokantisme qui n'arrive à la signification qu'en passant [11] par les valeurs. On peut se demander également jusqu'à quel point la pratique de Max Weber se conforme toujours aux schémas de la théorie. Les relations causales prennent-elles autant de place dans sa pratique que dans la théorie ? L'essentiel des études de sociologie religieuse n'est-il pas l'exploration des différents systèmes de croyances et de pensées, aboutissant à montrer l'entrecroisement des idées et des institutions, le lien entre valeurs religieuses et attitude sociale, bien plutôt qu'à isoler l'efficace propre des divers éléments ? L'essentiel de Wirtschaft und Gesellschaft n'est-il pas la mise à jour de la structure intelligible, propre aux divers types de pouvoir et d'économie? Or il ne suffit pas que la relation compréhensive soit intelligible pour être vraie en telle circonstance donnée, mais la vérification n'a pas grand-chose de commun avec la coïncidence de la relation compréhensive et de la relation causale. 
Max Weber en effet simplifiait, dans la théorie, et la multiplicité des relations intelligibles que dégage l'historien ou le sociologue, et la complexité des rapports entre compréhension et explication ou encore entre la relation immanente au donné et la relation élaborée grâce à des calculs rétrospectifs de probabilité. Il y a des types divers d'intelligibilité depuis la conduite conforme aux lois de l'économie abstraite jusqu'à la conduite conforme à la logique du ressentiment, en passant par la conduite dictée par l'interprétation d'une certaine doctrine de salut. La vérification ne signifie pas la même chose selon qu'il s'agit de l'une ou de l'autre de ces relations intelligibles, que Max Weber avait tendance à traiter simultanément dans sa méthodologie de la compréhension. Rectifications et compléments n'entraîneraient pas de modifications décisives, en ce qui concerne le thème principal de notre étude. En certains cas, au contraire, en matière de théorie économique ou d'explication d'un événement par confrontation avec un schéma, on insisterait, me semble-t-il, sur l'objectivité et l'universalité accessibles. Mais on ne serait pas obligé à une révision fondamentale.

Prenons le cas de la compréhension. L'amour et la haine, a-t-on dit, sont les vrais ressorts de la compréhension. Il est vain de recommander l'objectivité, si l'on entend par là l'indifférence aux valeurs, [12] quand il s'agit des hommes, d'aujourd'hui ou d'hier, et de leurs œuvres, bénies ou maudites. On n'arriverait pas à saisir l'âme profonde de ces êtres disparus si l'on n'éprouvait à leur égard des sentiments comparables à ceux qu'éveillent les vivants. Max Weber n'aurait probablement pas fait de grandes difficultés pour reconnaître la part de vérité que contiennent ces remarques, mais il en aurait restreint la portée à la psychologie de la recherche plutôt qu'à la logique de la science. Il aurait maintenu et l'impératif moral de tendre à la compréhension sine ira et studio et le devoir de viser l'existence ou les œuvres comme des objets à connaître et non pas comme des valeurs à apprécier.

Quant aux propositions économiques ou sociologiques, dont la vérité, à l'intérieur d'une société particulière ou, à un degré supérieur d'abstraction, dans toute société, serait moins liée au présent que ne le suggère Max Weber, elles ne changent pas essentiellement l'analyse du choix et de l'action. Ces vérités sont partielles, les valeurs auxquelles l'homme se réfère sont multiples, il est rare que les conséquences prévisibles d'une mesure soient conformes à toutes nos valeurs et agréables à tous les individus. Ce n'est pas la subjectivité et la 
relativité de la science qui fondent la nécessité du choix, mais le caractère partiel des vérités scientifiques et la pluralité des valeurs.

L'objection, selon laquelle la phénoménologie du choix politique serait fausse parce que Max Weber aurait utilisé des schémas désuets, le fait et la valeur, le moyen et la fin, passe, me semble-t-il, à côté de l'essentiel. On admettra que le fait auquel on oppose la valeur n'a pas été construit sans référence de l'historien à des valeurs. Les valeurs ne sont pas affirmées ou inventées, en dehors de ces échanges incessants entre l'individu et le milieu, échanges qui sont une des caractéristiques de l'historicité de l'homme. De même, la fin prochaine devient le moyen d'une action ultérieure, comme le moyen actuel peut être la fin d'une autre action. Bien plus, il est douteux que l'attitude adoptée par la personne soit réductible à une telle distinction. En adoptant une certaine perspective sur l'histoire, on est tout près d'adhérer à un parti, de souscrire à une technique d'organisation et d'action. La perspective globale [13] est autant choix des moyens que de la fin.

Max Weber n'ignorait pas qu'il en va ainsi. Le marxiste s'imagine qu'il possède l'interprétation vraie d'un mouvement de l'histoire à la fois nécessaire et souhaitable et que cette interprétation implique l'adhésion à un parti et à une méthode d'action. L'expérience a prouvé que cette philosophie ne supprimait ni le doute sur les partis ni le doute sur les méthodes. Nuls ne se combattent aussi impitoyablement que ceux qui se réclament du même maître. En dehors même de cette expérience, Max Weber aurait nié qu'une philosophie de l'histoire pût à la fois annoncer un avenir certain et dicter impérativement une attitude. La prévision de l'avenir suppose le déterminisme et celui-ci, orienté vers un terme à venir, est aussi partiel et plus probabiliste encore que dans l'usage rétrospectif. Les caractères de la société future que dans le meilleur cas nous sommes susceptibles de connaître, à l'avance laissent place à des jugements de valeur contradictoires parce qu'ils ne satisfont pas toutes les aspirations de l'homme. Nous ne sommes jamais moralement contraints d'aimer ce que la science nous annonce. Libres de hâter ou de retarder l'évolution qu'on nous baptise inévitable, nous nous retrouvons devant le choix d'une perspective globale, dans une situation comparable à celle qui confronte l'homme d'action en une conjoncture singulière : nous observons des faits, nous souhaitons des fins déterminées, et nous choisissons à nos risques et périls, sans avoir le droit d'invoquer ni une totalité qui ne nous est pas 
accessible, ni une nécessité qui n'est que l'alibi de notre résignation ou de notre foi, ni une réconciliation des hommes et des dieux, qui n'est qu'une idée située à l'horizon de l'histoire.

Ce qui est en question, ce n'est donc ni le schéma moyen-fin, effectivement trop simple, ni la distinction faits-valeurs, dont la portée philosophique est discutable. Pour réfuter la pensée de Max Weber, il faudrait démontrer soit que la science nous découvre la vérité de l'histoire totale, soit qu'elle connaît à l'avance un avenir prédéterminé, soit qu'elle promet la résolution des conflits entre les collectivités et les valeurs.

[14]

\section{- $\mathbf{0}$ -}

Max Weber avait à cœur de démontrer que la science, bien qu'elle aboutit à dépouiller le monde de ses charmes, bien qu'elle fût par essence inachevée, avait un sens et méritait qu'on s'y consacrât.

Il se battait sur deux fronts, contre ceux qui risquaient de corrompre la pureté de la pensée rationnelle en y mêlant des prises de position politiques ou des effusions sentimentales, contre ceux qui faussaient la signification de la science en lui prêtant la capacité de saisir le secret de la nature ou de l'homme. La défense et illustration de la science revêt, dans les discours de Max Weber, un ton pathétique parce qu'on y sent l'écho amorti d'une nostalgie et l'impatience d'un homme d'action. Nostalgie des temps où la connaissance n'était pas seulement un chaînon d'une chaîne sans fin, mais plénitude et accomplissement. Impatience d'un homme d'action qui demande à la science la connaissance des moyens et des conséquences, mais qui sait à l'avance qu'elle ne le délivrera pas de l'obligation de choisir, parce que les dieux sont multiples et les valeurs contradictoires.

Die Entzauberung der Welt durch die Wissenschaft, le désenchantement du monde par la science continue. Moins que jamais la science authentique, qu'il s'agisse de la physique ou de la sociologie, !Ions donne de l'univers, cosmique ou humain, une image achevée, dans laquelle on pourrait lire notre destin on notre devoir. Mais deux phénomènes sont intervenus qui font peser sur les universités, en Europe, une sourde angoisse. Les moyens de détruire, mis par les progrès de la 
science à la disposition des chefs civils ou militaires, sont devenus à ce point démesurés que les savants, responsables de ces découvertes et de leur application, s'interrogent sur leurs responsabilités. Nous connaissons, en notre siècle, des tyrannies positives, qui ne se bornent pas à imposer aux savants un serment de fidélité à l'État - ce qui pourrait être odieux pour les hommes, mais non mortel pour la science pour qui le fait de chercher et de dire la vérité objectivement est insupportable. Ces tyrannies prétendent imposer aux universités une doctrine qui se veut totale et qui [15] n'est qu'une dérisoire caricature des grandes synthèses religieuses du passé.

Sur le premier phénomène, je ne dirai que quelques, mots. Tout accroissement de la capacité de produire a été accompagné, depuis quelques siècles par un accroissement de la capacité de détruire. La nouveauté est uniquement dans l'ordre de grandeur. C'est l'augmentation de qualité qui crée la différence de qualité. Le savant, individuellement, ne saurait guère prendre de précautions contre l'exploitation de ses travaux par l'industrie de guerre. Collectivement, s'il se dérobe au service de l'État, il favorise d'autres États, ceux-là mêmes qui réduisent le plus les libertés des individus. Les associations de savants, dès qu'elles discutent de la paix ou de la guerre, sont des associations politiques et non scientifiques. Leurs appels seraient plus convaincants s'ils ne manifestaient souvent, en matière de diplomatie, une naïveté égale à l'autorité qu'on attribue, de confiance, à leurs auteurs en fait de physique nucléaire.

Plus important pour nous, qui nous occupons de science humaine, est le second phénomène, la menace que font peser sur les universités et sur la science certains régimes politiques. On a connu la "mathématique aryenne », on connaît aujourd'hui un État qui tranche la querelle scientifique relative à l'hérédité des caractères acquis ou à la théorie mendélienne. Les deux exemples sont de caractère différent. Après tout, je ne pense pas qu'il y ait eu beaucoup de mathématiciens allemands qui aient pris au sérieux la distinction entre « mathématique aryenne » et "mathématique enjuivée », ni beaucoup de physiciens qui aient tenu Einstein pour disqualifié à cause de sa race on de sa religion. Mais il était grave qu'en un pays comme l'Allemagne tant de savants fussent obligés de tolérer silencieusement cette comédie indigne comme s'ils la prenaient au sérieux et, par là même, de porter 
une atteinte grave à cette République internationale des esprits qui est la communauté, naturelle et nécessaire, des savants.

Rien n'est plus faux que l'idée selon laquelle le savant travaillerait seul, livré à sa fantaisie on à son génie. Les mathématiciens, les physiciens, les biologistes, séparés par les frontières, dispersés à [16] travers la planète, sont tenus ensemble par les liens, invisibles et puissants, d'une communauté de recherches, de règles intellectuelles, informulées mais obligatoires. Les problèmes à résoudre leur sont fournis par l'état d'avancement des sciences (ainsi s'explique la fréquence des découvertes simultanées). Une conception implicite et quasi spontanée de ce qu'est une vérité les amène à écarter tels types de solutions, à accepter les critiques réciproques, à s'enrichir par les échanges.

Certaines spéculations, mathématiques ou physiques, sont devenues à tel point subtiles que la République de la Science ne comporte plus, à travers le monde, que quelques membres. Ces spéculations n'en sont pas moins en droit universelles, adressées à tous les esprits capables de les saisir et rebelles à toute instruction qui serait extérieure à leur essence. Mon ami Cavaillès, mathématicien et philosophe, écrivit un ouvrage de logique mathématique alors qu'il était pourchassé par la police. Français et soldat, il se battait contre l'occupant. Logicien, il restait disciple de Cantor, de Hilbert ou de Husserl. Au juge d'instruction il témoignait de l'admiration qu'il gardait pour le meilleur de la culture allemande. L'idée ne l'effleurait pas que les conflits collectifs, ceux même qu'il assumait au point d'y sacrifier sa vie, pussent pénétrer dans le sanctuaire de la pensée fidèle à sa vocation, c'est-à-dire à la recherche de la Vérité.

Quand un État ou un parti prétend dicter à la science ses objets d'études ou les lois de son activité, quand il prétend exclure tel individu ou telle nation, quand il va jusqu'à arbitrer des controverses qui relèvent de l'expérience ou du raisonnement, il ne suffit pas d'évoquer, selon une formule banale, l'oppression des individus par la collectivité. Il s'agit de l'intervention illégitime d'une collectivité politique dans l'activité d'une collectivité spirituelle, il s'agit, en d'autres termes, du totalitarisme, saisi à sa racine même. L'invention la plus redoutable du totalitarisme, c'est précisément de subordonner les œuvres multiples dont l'homme est créateur à la volonté exclusive d'un parti et parfois d'un homme. G. Simmel, dans sa Sociologie, a décrit de brillante ma- 
nière la pluralité des cercles sociaux auxquels chacun de nous appartient, et il [17] voyait dans cette pluralité la condition de la libération progressive des individus. Ce souvenir nous permet de juger les tentatives de totalitarisme pour ce qu'elles sont : des efforts proprement réactionnaires pour ramener les sociétés au stade primitif où les disciplines sociales tendaient à embrasser tous les individus et les individus tout entiers.

Certes, on observe une détermination partielle de la science par des facteurs sociaux, historiques, éventuellement raciaux. Il n'est pas inconcevable que telle race soit mieux douée qu'une autre pour une certaine sorte de travaux ou encline à une certaine manière de penser le monde, encore qu'il soit presque impossible d'isoler l'action de la race. La plupart des généralisations, en cette matière, ont été démontrées fausses. Les autres sont indémontrables. Mais quel que soit le degré de la détermination de la science - des questions qu'elle se pose, des idées philosophiques dans lesquelles elle s'exprime - par des circonstances extérieures, ceux qui invoquent cette détermination de fait pour justifier l'orientation autoritaire de la science par les pouvoirs publics commettent une erreur fatale.

Les savante sont, en même temps les hommes d'une société particulière, d'une époque donnée. L'orientation et le style des recherches sont marqués par le caractère des hommes et non pas des seuls savants, car les uns ne sont jamais rigoureusement séparables des autres. Malgré tout, la différence demeure fondamentale entre l'influence que le milieu exerce de lui-même sur la science, en passant par la spontanéité des savants, et celle que les chefs politiques exerceraient s'ils s'arrogeaient le droit de fixer à la science ses objectifs, puis ses méthodes, finalement ses résultats. Dans le premier cas, la communauté de la science continue d'obéir, pour l'essentiel, à ses lois spécifiques. Dans l'autre, elle abdiquerait son autonomie et mettrait en péril, du même coup, sa vocation et ses progrès ultérieurs.

En Grande-Bretagne même on a, au lendemain de la guerre, discuté de l'indépendance de la science. Quelques savants, impressionnés par l'exemple soviétique, souhaitaient l'établissement d'un plan de recherches, avec répartition rationnelle des ressources matérielles et humaines entre les différents laboratoires, chacun recevant sa mission particulière. [18] Les savants britanniques ont rejeté cette conception pragmatique; ils ont dénié à l'État le droit d'indiquer aux savants ce 
qu'ils devaient chercher. L'abandon ou la subordination de la recherche théorique serait non seulement fatal au progrès de la technique (on ne sait jamais à l'avance quelle théorie aboutira à une application) mais aussi la première étape d'une abdication de la communauté scientifique, aliénant son autonomie. La disparition des biologistes mendéliens ou l'obligation pour les physiciens d'envelopper leurs résultats dans le jargon du matérialisme dialectique illustrent ce que pourrait être l'étape finale.

Les sciences sociales sont autrement menacées par les totalitarismes que les sciences naturelles. Les tyrans ont besoin de ces dernières pour accumuler les moyens de puissance : il est une limite, marquée par le souci de l'efficacité, à leurs interventions. On oblige les physiciens à se réclamer du matérialisme dialectique, on ne leur dicte pas leurs équations. Au bout du compte, on est suprêmement intéressé au développement de la physique nucléaire. Rien n'empêche de tenir et d'imposer les théories marxistes de la plus-value et du salaire pour vérités définitives - il n'en résulte pas de dommage irréparable pour la planification concrète. L'orthodoxie, n'étant pas nuisible, au moins directement, à l'efficacité de la gestion, devient un instrument de l'unanimité artificielle que les despotismes tiennent pour une garantie de stabilité.

La résistance des sciences sociales à l'intrusion de la politique a toujours été plus difficile que celle des sciences naturelles. Il y a incontestablement, dans le passé, une solidarité entre certains modes d'analyse et certaines préférences politiques ou certaines conceptions philosophiques. Rien n'est plus facile et plus tentant, en économie politique, que la confusion entre les schémas idéaux et la réalité. On prête à celle-ci les mérites qui n'appartiennent, en rigueur, qu'à ceuxlà. S'il est vrai que l'état d'équilibre, défini par la théorie classique du marché, comporte la maximation du produit social et l'emploi le meilleur des ressources, il ne s'ensuit pas que les économies concrètes que l'on appelle libérales, c'est-à-dire partiellement libérales, soient les meilleures ni qu'il convienne de laisser [19] jouer les mécanismes imparfaits du marché, sous prétexte que, si ces mécanismes étaient réalisés dans leur perfection, ils apporteraient la solution la meilleure. Karl Mannheim a donné le nom de Wissenssoziologie à la discipline qui s'efforce d'élucider les diverses modalités des relations entre les circonstances historiques et les édifices intellectuels. 
Que les sciences sociales ne partent jamais de la table rase, que la position des problèmes soit suggérée par les événements, que la méthode ne soit pas indépendante de la philosophie ou du milieu historique, que souvent les résultats soient influencés par les intérêts des nations et des classes, nous ne songeons pas à le nier. Il n'en serait pas moins fatal d'en tirer la conclusion que les sciences sociales ne sont que des idéologies de classes ou de races et que l'orthodoxie imposée par un État totalitaire ne diffère pas en nature de la libre recherche des sociétés pluralistes. Il existe, quoi qu'on en dise, une communauté des sciences sociales, moins autonome que la communauté des sciences naturelles mais malgré tout réelle.

Quelles sont règles constitutives de cette communauté des sciences sociales?

$1^{\circ}$ D'abord, l'absence de restriction dans la recherche et l'établissement des faits eux-mêmes, le droit de présenter les faits bruts et de les distinguer des interprétations. Sans doute peut-on soutenir, en rigueur philosophique, qu'il n'y a pas de fait historique qui ne soit construit et ne comporte, par conséquent, sélection et interprétation. Dans la pratique, les distinctions gardent leur portée. Il est vrai ou il est faux que Trotsky a pris une part considérable à l'organisation de l'armée rouge, il est vrai ou il est faux que Zinoviev ou Boukharine ont comploté l'assassinat de Staline, il est vrai ou il est faux que Wall Street manipule les hommes politiques américains et organise une croisade anti-soviétique. Tout État totalitaire met certains faits hors la loi parce qu'ils ne rentrent pas dans le schéma officiel. Tout État totalitaire pousse jusqu'à l'absurde la solidarité entre le fait et l'interprétation.

$2^{\circ}$ Ensuite, l'absence de restriction au droit de discussion et de critique, appliquées non pas seulement aux résultats partiels, mais aux fondements [20] et aux méthodes. Les connaissances sociales s'élèvent au niveau de la science dans la mesure où elles s'accompagnent d'une conscience exacte de leur portée, et aussi de la limite 
'de leur validité. En économie politique, par exemple, la théorie progresse en construisant de nouveau modèles, mais aussi en reconnaissant les conditions précises dans lesquelles tel modèle, connu depuis longtemps, s'applique. La théorie générale de Keynes doit son originalité à de multiples facteurs : la situation qui a posé le problème (chômage dit permanent), l'effort pour retrouver, à partir d'un schéma inédit, la théorie classique comme cas particulier valable dans l'hypothèse $\mathrm{du}$ plein emploi, la détermination de quelques faits concrets (fixité des salaires nominaux) comme nécessaires, une psychologie de l'entrepreneur différente de la psychologie rationnelle du sujet économique de la théorie traditionnelle, etc. L'apport, la signification de la théorie générale ne se découvre qu'à celui qui considère simultanément ces divers éléments, de fait et de méthode. La théorie de la connaissance est, en matière de science sociale, inséparable de la connaissance elle-même. Toute doctrine, qu'elle soit libérale ou marxiste, qui érige en dogmes des propositions ou des schèmes, dont la signification est équivoque et la portée imprécise, glisse de la science à la mythologie. La conscience critique est, en sociologie on en économie politique, constitutive de la conscience scientifique elle-même.

$3^{\circ}$ Enfin, l'absence de restriction au droit de désanchanter le réel. Entre l'idée d'un certain régime et le fonctionnement de ce même régime, entre la démocratie que nous avons tous rêvée sous la tyrannie et le système des partis tel qu'il s'est instauré dans l'Europe occidentale, le décalage n'est pas mince. Mais cette déception est pour une part inévitable. Toute démocratie est oligarchie, toute institution est imparfaitement représentative, tout gouvernement qui doit obtenir l'assentiment de groupes ou de personnes multiples agît lentement et doit tenir compte des sottises ou des égoïsmes humains. La première leçon qu'un sociologue doit transmettre à ses étudiants, quitte à décevoir leur ardeur de croire et de servir, est qu'il n'y a jamais eu un régime parfait.

[21]

Il est rare qu'un savant use de ces trois libertés, simultanément et sans limites, il serait presque inhumain qu'il le fit. La communauté des sciences sociales a précisément pour fonction de créer, par le dialogue et par la critique mutuelle, l'équivalent de ces trois libertés. L'autre me 
montre la part d'interprétation que comportent les faits bruts que je crois constater ou les conséquences fâcheuses des institutions dont j'étais tenté de n'apercevoir que les mérites. C'est dans et par l'ensemble de la communauté que les sciences sociales tendent à n'écarter aucun fait, à n'épargner la critique à aucune valeur, à accumuler tout à la fois les connaissances et les doutes, en précisant impitoyablement les conditions extérieures et les hypothèses préalables auxquelles est suspendue la vérité des propositions générales.

Du même coup, on aperçoit à quelle fin répond cette libre communauté des sciences sociales et pourquoi tant de gouvernants la redoutent. Seule la science critique empêche que l'histoire on la sociologie glisse de la connaissance positive à la mythologie, mais beaucoup de régimes ne souhaitent pas empêcher ce glissement.

Les événements de l'histoire ont tout ce qu'il faut pour être transfigurés en mythologie. Ils sont proches de nous, ils sont humains : nous sommes inévitablement tentés de les attribuer à la volonté claire et résolue de quelques personnages, individus ou groupes, qui deviennent angéliques ou monstrueux en raison même du bien et du mal démesurés qu'ils sont censés répandre. La plupart des hommes du $\mathrm{XX}^{\mathrm{e}}$ siècle ne savent pas comment expliquer les phénomènes qui auraient été naguère considérés comme miraculeux, le vol du plus lourd que l'air, la transmission à distance du son et de l'image, mais ils savent que ces phénomènes s'expliquent rationnellement. L'électricité n'est une fée que pour les enfants. Le capitalisme, le communisme, Wall Street sont des, démons pour des millions de grandes personnes. L'histoire incite à la mythologie par sa structure même, par le contraste entre l'intelligibilité partielle et le mystère du tout, entre le rôle évident des volontés humaines et les démentis non moins évidents que les événements leur infligent, par l'hésitation du spectateur entre l'indignation, [22] comme si nous étions tous et chacun responsables de ce qui se passe, et l'horreur passive, comme si -nous étions en présence d'une fatalité inhumaine.

Les concepts de la science deviennent, si nous n'y prenons garde, les personnages de la mythologie. Il suffit de confondre nos schémas avec le réel, d'oublier les sens multiples des phénomènes dispersés que désigne un terme comme capitalisme on socialisme, et bientôt la substitution s'est faite. Nous ne sommes plus en présence d'hommes et d'institutions, de significations immanentes à la conduite de ceux-là et 
à la structure de celles-ci, mais d'une force mystérieuse, qui a gardé la signification que nous attachions au mot, mais perdu le lien avec les faits. L'Histoire, désormais avec une majuscule, devient le lieu de combats grandioses entre Idées. Les sciences historiques ne suppriment pas le mystère des ensembles supra-individuels, mais elles le dépoétisent. Le dialogue des savants sur le devenir des collectivités n'enseigne ni le scepticisme ni l'irrespect, mais il interdit de diviniser les choses temporelles, il ramène sur la terre ceux des hommes ou des régimes qu'on élève au-dessus du sort commun.

Les gouvernements despotiques ne peuvent guère ne pas prendre ombrage des sciences sociales, dès lors que celles-ci ne se bornent pas à l'étude des techniques administratives et vont jusqu'au bout de leur mission.

Même si, par prudence on par nécessité, le sociologue ou l'historien s'abstient d'étudier les caractères des césarismes anciens et modernes, même s'il borne son étude aux régimes différents de celui sous lequel il vit, l'étudiant ne peut pas ne pas comprendre que la même méthode devrait s'appliquer à ses maîtres et qu'elle enlèverait à ces derniers l'auréole de perfection ou d'infaillibilité. Par crainte d'être accusés d'anti-démocratisme, ne nous arrêtons pas devant l'analyse des institutions parlementaires telles qu'elles fonctionnent à l'heure présente en Europe.

La science ne nous dira pas qu'il faut être favorable à la démocratie, ni que celle-ci est en tant que telle supérieure aux autres formes de gouvernement praticables en notre siècle. Elle montre les risques illimités que comportent les régimes de [23] parti unique pour certaines valeurs que le professeur, attaché à la tradition séculaire des universités, tient pour sacrées. Elle montre quelles relatives garanties le système des partis multiples donne et d'un certain respect des droits personnels et du caractère constitutionnel des pouvoirs et de leur exercice. Elle montre aussi quels risques sont immanents à ce régime : instabilité de l'exécutif, dans le cas où aucune majorité se dégage, décomposition sociale lorsque les luttes de partis ou de classes dépassent une certaine violence, paralysie des gouvernants lorsque tous les groupes, tous les intérêts particuliers réussissent à plaider trop bruyamment leur cause. 
On craint souvent que la science politique ne soit redoutable pour les démocraties parce qu'elle les montre telles qu'elles sont, dans leur inévitable et bourgeoise imperfection. Je ne crois guère à ce danger. Ne l'oublions pas : la démocratie est le seul régime, au fond, qui avoue, que dis-je, qui proclame que l'histoire des États est et doit être écrite non en vers mais en prose.

Qu'il s'agisse de science ou de politique, Max Weber visait le même but : dégager l'éthique propre à une activité qu'il voulait conforme à sa finalité. Le savant doit refouler les sentiments qui le lient à l'objet, les jugements de valeur qui surgissent spontanément en lui et commandent son attitude à l'égard de la société, celle d'hier qu'il explore et celle d'aujourd'hui qu'il désire, quoi qu'il en ait, sauvegarder, détruire ou changer. Accepter le caractère indéfini de la recherche positive et, au profit d'une enquête dont on ignore le terme, désenchanter le monde de la nature et celui des hommes, tel est le pathétique qu'il découvrait à ses auditeurs et qu'il les sommait d'assumer au nom du choix qu'ils avaient fait de la carrière scientifique.

Le pathétique de l'action était lié, à ses yeux, à l'antithèse des deux morales, morale de la responsabilité et morale de la conviction. Ou bien j'obéis à mes convictions - pacifistes on révolutionnaires, peu importe - sans me soucier des conséquences de mes actes, ou bien je me tiens pour comptable [24] de ce que je fais, même sans l'avoir directement voulu, et alors les bonnes intentions et les cœurs purs ne suffisent pas à justifier les acteurs.

En même temps, Max Weber ne se lassait pas de souligner le décalage entre les projets des hommes et les suites de leurs actes. Ce qu'une génération a librement voulu est, pour la génération suivante, destin inexorable. Les puritains choisissaient d'être hommes de métier, les hommes d'aujourd'hui sont contraints de l'être. Nous ne sommes pas tentés de mettre en doute le perpétuel écart entre ce que les hommes souhaitent et ce qu'ils subissent, quand nous évoquons les espoirs de Lénine et que nous observons la réalité du stalinisme, quand nous nous souvenons de la foi qui soulevait tant de jeunes Allemands 
en 1932 ou en 1933 et que nous nous rappelons certaines horreurs du nazisme. Oui, l'histoire est la tragédie d'une humanité qui fait son histoire, mais qui ne sait pas l'histoire qu'elle fait. L'action politique n'est rien si elle n'est l'effort inlassable pour agir dans la clarté et n'être pas trahie par les suites des initiatives qu'elle a prises.

La morale de l'homme d'action est bien celle de la responsabilité. Mais qu'on ne prenne pas une telle affirmation à la légère. Elle exclut la soumission aux règles d'une éthique formelle dans le style kantien ou aux impératifs sublimes du Sermon sur la montagne. L'État est l'institution qui possède, dans une collectivité donnée, le monopole de la violence légitime. Entrer dans la politique, c'est participer à des conflits dont l'enjeu est la puissance - puissance d'influer sur l'État et par-là même sur la collectivité. Du même coup, on s'oblige à se soumettre aux lois de l'action, fussent-elles contraires à nos préférences intimes et aux dix commandements, on conclut un pacte avec les puissances infernales, on se condamne soi-même à la logique de l'efficacité.

À qui en avait Max Weber à l'époque où, au lendemain de la première guerre mondiale, il écrivait Politik als Beruf? Il visait surtout, nie semble-t-il, deux catégories d'hommes, ceux que nous appellerions en français les pacifistes d'inspiration chrétienne et les révolutionnaires de principe. Aux premiers, auxquels il ne refusait nullement l'estime personnelle, il reprochait de ne pas tenir compte des [25] conséquences de leurs propos ou de leurs écrits. Charger son pays de toutes les responsabilités, ne contribue pas à rétablir la paix entre les pays. En affaiblissant la position morale du vaincu, on prépare un traité dont la rigueur et l'injustice empêcheront l'apaisement et la réconciliation. Aux seconds, il reprochait de transfigurer un but légitime - une transformation du régime économique et social - en une valeur absolue de telle sorte que la révolution, aux yeux de ses fidèles, ne saurait jamais être payée trop cher.

Le dialogue avec les pacifistes d'inspiration chrétienne a, je crois, perdu aujourd'hui sa signification. Il n'y a plus guère de tels pacifistes en un inonde où la guerre est devenue quasi permanente. Ceux qui se donnent pour tels camouflent la plupart du temps une prise de position plus politique que spirituelle. Plus fort serait aujourd'hui le pacifisme par réflexion, la conviction raisonnable que la guerre moderne est, pour ceux qui en sont les victimes, dont le territoire sert de champ de 
bataille, en tout état de cause une catastrophe. Mais cette conviction ne deviendrait facteur de paix qu'à la condition d'être partagée par tous les hommes d'État, par tous les peuples. Or les communistes sont tenus par leur doctrine à croire que la phase historique, qu'ils baptisent révolution mondiale, sera remplie de luttes gigantesques, dont le socialisme universel sera l'aboutissement nécessaire. Pour une telle fin, même une guerre atomique ne serait pas un prix trop élevé.

Quant aux révolutionnaires, contre lesquels se dressait Max Weber, ils étaient surtout des idéalistes, proches les uns des pacifistes d'inspiration chrétienne, les autres des anarchistes ou des utopistes. Aujourd'hui, les révolutionnaires sont des techniciens de la subversion et de la tyrannie qui pourtant continuent de penser dans les cadres du millénarisme, ils prêtent à un événement - la révolution - une valeur unique, qui la met en dehors du train immémorial de l'histoire humaine. Rien ne serait possible jusqu'au jour de cette conversion de l'Histoire, tout serait possible à partir de ce jour. Aucun moyen ne saurait être écarté pour atteindre cet objectif suprême, d'ailleurs historiquement inévitable.

[26]

On imagine sans peine la réponse de Max Weber à une telle philosophie. Il se peut, aurait-il dit, que les sociétés occidentales évoluent nécessairement (en termes plus précis : probablement) vers un régime de propriété collective ou de planification *. Mais on ne saurait postuler à l'avance qu'un seul et même parti doive nécessairement accomplir cette transformation dans tous les pays. Quant aux avantages et inconvénients de ce régime, on en peut discuter, mais la science ne permet pas d'affirmer ni même de croire que les avantages en sont tels que les traits séculaires des sociétés humaines en seront décisivement modifiés.

Une telle argumentation ne toucherait pas les croyants, mais elle les ferait apparaître sous leur vrai jour : ils se réclament de la science, ils sont les fidèles d'une foi qui se drape dans les oripeaux tantôt d'une science vieillie, tantôt d'une philosophie vulgarisée. Max Weber, que ses études auraient dû inviter aux vastes perspectives sur l'histoire, en

Il y a trente ans, Max Weber n'en était pas convaincu. Les tendances nécessaires de l'évolution étaient pour lui la rationalisation et la bureaucratisation, non pas un type donné de propriété ou de régulation. 
avait tiré surtout une leçon de modestie. Il ne s'interrogeait pas sur la société d'après-demain, mais sur les tâches d'aujourd'hui. Cette tâche était, il y a trente ans, la reconstruction de la politique et de l'État.

Certaines de ses analyses se rapportent à une situation historique et ont perdu une part de leur actualité. Il était obsédé par son hostilité à l'égard de l'empereur auquel il imputait une responsabilité majeure dans la catastrophe allemande. Il considérait comme une des causes essentielles de la faillite de l'empire wilhelmien le recrutement des chefs politiques parmi les fonctionnaires. La « parlementarisation », le transfert au parlement de responsabilités effectives offrirait aux personnalités énergiques, combatives, animées par la volonté de puissance et de lutte, une meilleure chance d'accéder au pouvoir.

Max Weber suivait, à travers les siècles, le développement d'une catégorie sociale, d'un type d'homme, qu'il appelait politicien professionnel, celui qui tire sa subsistance de la politique, qui vit [27] par en même temps que pour elle. Les clercs, les lettrés, la noblesse de cour, le patriarcat anglais, les juristes ont tenu, selon les siècles et les pays, la première place, les relations variables avec les nations, entre le monarque, la noblesse traditionnelle et la bourgeoisie déterminant les alliances ou les conflits entre ces divers groupes. Sans rappeler le détail de ces analyses, justement célèbres, j'insisterai sur un point, que Max Weber lui-même soulignait, à savoir le contraste décisif entre deux types de partis, les partis de notables et les partis de masses, les juristes ou avocats, les fonctionnaires de l'État ou des partis, les notables constituant les principaux types de politiciens professionnels à notre époque.

Comment se présente aujourd'hui la situation? L'opposition des partis de notables et des partis de masses, qui s'est presque effacée dans certains pays - le parti conservateur est devenu, en Grande-Bretagne, un parti de masses presque au même titre que le parti travailliste - subsiste, me semble-t-il, aussi bien en Allemagne qu'en France. Les radicaux-socialistes, les indépendants ne sont pas des partis de masses au sens où le sont la S.F.I.O. et même jusqu'à un certain point le M.R.P. Le C.D.U. conserve, nie semble-t-il, quelques caractères du parti de notables, en dépit des tentatives faites pour le transformer en organisation de niasses. Celle-ci devient ou non nécessaire selon le degré d'urbanisation de la société et aussi selon le mode de scrutin. Au cours de la phase actuelle, en Europe continentale, la diffé- 
rence de structure entre partis de droite et partis de gauche, qui tend à s'atténuer, ne doit pas disparaître entièrement.

Avec la différence des structures de partis va la différence de recrutement des chefs politiques. Le recrutement des chefs est, dans tous les régimes politiques, dans les démocraties plus que dans tout autre, le problème décisif, celui qui commande le succès on l'échec. En Allemagne, les principaux leaders appartiennent encore à une génération préhitlérienne, dont la plus grande partie de l'existence s'est déroulée soit sous l'empire wilhelmien, soit dans la République de Weimar. En France, les leaders sont en majorité des survivants de la III ${ }^{\mathrm{e}}$ République, promus depuis 1945 au premier [28] rang après avoir tenu longtemps un rôle secondaire, auxquels se sont ajoutées quelques personnalités de la Résistance ou du mouvement gaulliste. N'est presque pas représentée en France là catégorie, importante en Allemagne) de ceux qui Ont fait carrière en tant que fonctionnaires de partis ou de syndicats.

La difficulté, pour le professeur qui veut entrer dans la politique, vient de la discipline et des doctrines des partis. Il n'y a pas un seul sociologue ou économiste qui soit susceptible de prendre au pied de la lettre le programme d'aucun parti politique dans aucun pays du monde, à aucune époque. Il n'y peut adhérer, en mettant les choses au mieux, qu'en faisant un large usage de ce que l'on appelait, en matière religieuse, l'interprétation symbolique. Dans la lutte, il n'est pas d'exemple que l'opposition n'emploie des arguments injustes ou mensongers, arguments qui consistent à reprocher au Gouvernement de n'avoir pas remporté des succès qu'aucun autre n'aurait pu remporter ou d'avoir consenti des concessions qu'aucun autre n'aurait pu éviter. De là résulte chez le professeur de sciences sociales, qui veut être politiquement actif, une tension permanente.

Cette tension est plus on moins intense selon le degré de malhonnêteté caractéristique des discussions, degré variable selon les pays, variable également selon le degré de discipline exige des membres des partis, variable enfin selon les moments. Chacun de nous trouve sa réponse à ce problème personnel des rapports entre science et politique. Ceux qui participent aux travaux parlementaires ne sauraient s'offrir le luxe de la liberté intégrale. La vocation de la science est inconditionnellement la vérité. Le métier du politicien ne tolère pas toujours qu'on la dise. 
Peut-être la subordination au parti est-elle aujourd'hui moins grave qu'il y a vingt ans, grâce au scepticisme qui a rongé les croyances partisanes. Fort heureusement, ni les dirigeants ni les troupes de la socialdémocratie n'affectent même la fidélité au marxisme on le dogmatisme de la propriété collective. Les partis apparaissent ce qu'ils sont et ce qu'ils doivent être, des organisations qui visent à l'exercice du pouvoir, défendent certains intérêts et [29] promettent de gouverner en fonction de conceptions vagues et générales. Malheureusement, les partis compensent parfois ce qu'ils ont gagné en souplesse doctrinale par une violence accrue dans les controverses d'actualité.

Max Weber exprimait déjà des inquiétudes sur la qualité du recrutement démocratique. Ceux qui n'ont pas de fortune ne sauraient se lancer dans la carrière politique sans accepter, en même temps que les aléas du métier, l'insécurité financière. Il me semble que, dans l'Allemagne actuelle, la plupart des hommes politiques conservent et doivent conserver leur métier antérieur ou un métier secondaire, souvent un métier de fonctionnaire. Sont exclusivement politiciens professionnels, sans autre métier, les seuls fonctionnaires de partis. Le jeu parlementaire est à ce point formalisé, dépouillé de son caractère de lutte imprévisible, qu'il ne constitue plus un mode de sélection. C'est dans le parti plutôt qu'au parlement que l'on s'impose pour l'avancement. En France, la vie politique, ayant conservé plus d'instabilité ou de fantaisie, demeure plus imprévisible. De temps à autre, une, personnalité y fait carrière, non pas dans mais en marge des partis. On ne saurait dire que le style actuel de la politique, en Allemagne, favorise le surgissement de personnalités de premier ordre, de ces démagogues dont rêvait Max Weber, qui se dévouent à leurs œuvres en même temps qu'ils en vivent et combinent étrangement la passion lucide, le sens de la responsabilité et la mesure.

Mais peut-être Max Weber demandait-il trop aux hommes politiques des démocraties modernes. Il imaginait les meilleurs d'entre eux revêtus d'une est très vrai que les démocraties sont perpétuellement menacées par la décadence qu'entraînent l'anonymat des pouvoirs, la médiocrité des dirigeants, la passivité des foules sans âme. En des circonstances tragiques, quand la vie de la nation est en jeu ou que la constitution à besoin d'être restaurée, les peuples désirent suivre un homme en même temps qu'obéir aux lois. C'est alors que s'impose le démagogue, celui que la République romaine appelait le dictateur, que 
les auteurs politiques du passé appelaient le Législateur. Les régimes vivants font surgir, aux moments [30] critiques, les personnes capables de les sauver. En période tranquille, les chefs des démocraties sont d'honorables administrateurs, quelquefois de bons organisateurs, plus souvent des conciliateurs. Qu'ils aient aussi l'ampleur de vues, la clairvoyance, la passion lucide des grands hommes d'État, c'est là une bonne chance sur laquelle on ne saurait raisonnablement compter. 
Il y a quelques années, M. Leo Strauss a consacré un chapitre de son livre, Droit naturel et histoire ', à l'examen des conceptions de Max Weber. L'intention dernière de cette critique est de réduire, si l'on peut dire, Max Weber au nihilisme. En d'autres termes, M. Strauss veut montrer que, faute de jugement scientifique ou du moins rationnel sur les valeurs, l'homme est livré à l'arbitraire de décisions toutes également justifiables ou injustifiables. Mais, de son côté, il n'expose pas clairement ni quel est en lui-même le meilleur régime, ni comment la raison parvient à en préciser les caractères et à en fonder la validité universelle. On pourrait réduire la pensée de M. Strauss à un dogmatisme supra-historique, comme il réduit le relativisme de Max Weber au nihilisme. Nous ferons abstraction, provisoirement, des conséquences dernières des deux thèses pour considérer les objections que le philosophe, en quête de droit naturel, adresse au sociologue, soucieux d'établir une science objective et convaincu de l'irréductible diversité des époques humaines.

1. La première objection qu'avance $M$. Strauss pourrait être formulée en ces termes : l'interdiction de jugements de valeur est, en tant que telle, dénuée de signification parce que l'historien ou le sociologue [32] ne pourrait la respecter sans compromettre la qualité de sa science. "L'œuvre de Weber aurait été non seulement ennuyeuse mais absurde s'il n'eût parlé à tout bout de champ des vertus ou des vices intellectuels et moraux dans le registre approprié, celui de la

1 Publié en 1954 dans les Recherches en Sciences humaines. 
louange ou du blâme (page 67). » Et encore : « Weber, comme tous ceux qui ont un jour réfléchi sur la condition humaine, ne pouvait s'empêcher de parler d'avarice, de cupidité, de manque de scrupule, de vanité, de dévouement, de sens de la mesure et d'autres choses semblables, bref de prononcer des jugements de valeur. »

Sur ce premier point, on donnera, me semble-t-il, raison à $\mathrm{M}$. Strauss mais puisque, d'après son critique, Max Weber a violé la règle qu'il posait en théorie, il nous faut chercher pourquoi un savant, si soucieux de rigueur et de clarté, a pu méconnaître sa propre pratique.

Un historien ou un sociologue incapable de faire la distinction entre un vrai prophète et un charlatan serait du même coup incapable d'une compréhension authentique. Un historien de l'art qui ne distinguerait pas entre les tableaux de Léonard de Vinci et ceux de ses imitateurs laisserait échapper le sens spécifique de l'objet historique, c'est-à-dire la qualité de l'œuvre. Un sociologue qui mettrait dans le même sac Washington et Hitler, Boulanger et Charles de Gaulle, un politicien uniquement intéressé à la puissance et un homme d'État passionné de la grandeur de sa patrie, finirait par tout confondre sous prétexte de ne pas prendre parti.

Ces trois exemples suggèrent la même proposition générale : l'historien ne peut pas ne pas inclure, dans le récit ou l'interprétation des événements on des œuvres, des jugements de valeur dans la mesure où ceux-ci sont internes à l'univers d'action ou de pensée, constitutifs de la réalité elle-même. Pour éviter ces sortes de jugements de valeur, l'historien devrait s'en tenir à des propositions historiques au sens étroit du terme, analyser l'origine des tableaux en ignorant leur médiocrité ou leur excellence, constater la succession des styles sans établir de hiérarchie ni entre eux ni, à l'intérieur d'un style, entre les réalisations des créateurs et celles des imitateurs.

Max Weber, qui s'est soumis à cette exigence de [33] la recherche, n'en a pas pris conscience ou, du moins, s'est borné à maintenir la formule du rapport aux valeurs contre celle du jugement de valeur. Or cette distinction est étrangement sommaire.

Décréter beau ou laid un temple de l'Inde en se référant au canon de la beauté grecque serait un jugement de valeur du type que proscrit Max Weber au nom de l'objectivité des sciences sociales. Situer, sur une échelle hiérarchique, les divers accomplissements de l'architecture 
et de la sculpture de l'Inde est, en dernière analyse, inévitable : comment ne pas confronter aux temples du passé ceux que l'on élève aujourd'hui dans le style ancien et qui ne sont que la caricature d'un art qui a cessé de vivre ? De même, en fait de religion et de politique, on jugerait de la grandeur de l'homme de foi ou de l'homme d'action par rapport au sens qu'ils donnaient à leur prédication et à leurs entreprises. Max Weber n'a pas donné de définition de ce qu'il entendait exactement par valeur. Rien n'empêche de substituer au concept $v a$ leur les termes par lesquels concrètement se définissent les objectifs d'une conduite ou d'une œuvre, les règles auxquelles l'acteur ou le créateur doivent se soumettre.

Max Weber, de son point de vue propre, aurait pu consentir à cette rectification moins de sa pensée que dé l'expression qu'il en a donnée. Il n'aurait élevé des objections qu'en une phase ultérieure de l'argumentation : que l'on puisse et que l'on doive distinguer entre Léonard de Vinci et ses imitateurs, il l'aurait accepté, niais l'historien peut-il établir une hiérarchie entre les miniatures persanes et la peinture italienne, entre les statues de l'Elephanta et la statuaire de Phidias ? A l'intérieur d'un univers qui possède ses propres critères d'appréciation, l'historien ne peut pas ne pas apprécier sans fausser la compréhension du réel. Quand les critères sont foncièrement différents, quand les univers sont essentiellement autres, l'historien ne pourrait apprécier qu'en prenant parti et il cesserait du même coup d'être un savant.

L'examen de cette première objection, valable mais susceptible d'être intégrée à la pensée de Max Weber, nous mène à une deuxième objection, qui est aussi un deuxième thème de réflexion : quel est le sens de cette diversité des univers ?

[34]

2. L'acceptation d'une diversité radicale entre les différentes époques détruirait, en dernière analyse, la Portée de la sociologie historique elle-même. En effet, le sociologue ne peut comprendre une société sans user d'un schéma conceptuel. Or, s'il utilise son propre schéma conceptuel, il comprendra la société étrangère autrement que celle-ci ne s'est comprise elle-même et, du même coup, il en faussera la signification (à moins qu'il ne la comprenne mieux qu'elle ne s'est comprise elle-même). La compréhension de l'autre par rapport à notre 
système conceptuel est relative à celui-ci et, dans la mesure où ce système est changeant, notre compréhension est changeante comme lui ; bien loin d'être universellement valable, elle est liée à l'histoire et à son époque. Quand le sociologue tente d'établir une science comparative des institutions, ou bien il dispose d'un ensemble catégoriel, dont la validité dépasse les limites d'un temps, ou bien son œuvre est éphémère et, en dernière analyse, insignifiante.

L'objection que nous avons formulée dans des termes légèrement différents de ceux qu'emploie M. Strauss comporte une conclusion incontestable, que Max Weber lui-même acceptait : la science historique ou sociale est universellement valable, mais d'une universalité hypothétique. Elle est suspendue à des hypothèses initiales, choix de valeurs et rapport aux valeurs, qui ne s'imposent pas à tous les hommes et qui changent d'époque en époque. Mathématiques on physique, dira-t-on, ne valent, elles aussi, que pour ceux qui se soucient de cette sorte de vérité. Mais il y a une différence décisive entre sciences de la nature et sciences de la "culture », telles que les interprète Max Weber : une fois voulue la vérité mathématique ou physique, le devenir de ces sciences est accumulation. Même en cas de renouvellement théorique, les propositions d'hier trouvent place, à leur degré d'approximation, dans l'édifice d'aujourd'hui. En revanche si, d'époque en époque, les questions des historiens et des sociologues se modifient, l'homme du XXI siècle, même s'il veut une vérité objective, n'est pas tenu de s'intéresser aux questions que posait l'homme du $\mathrm{XX}^{\mathrm{e}}$.

Max Weber acceptait sans hésitation cette conséquence de ses propres principes, pour deux raisons, [35] l'une explicite, l'autre inexprimée. Les questions sont posées par le savant, disait-il, mais les réponses sont universellement valables, une fois les questions posées. D'autre part, sans le dire, il ne pouvait pas ne pas croire que les questions que, lui, posait au passé, l'homme des temps à venir continuerait à leur trouver un sens. Or cette raison inexprimée, bien plus que l'objectivité des réponses, fonde la validité des sciences historiques.

Ce que Max Weber appelait la question, ou le rapport aux valeurs, ne détermine pas seulement l'objet ou les limites de la recherche, il entraîne la mise en forme conceptuelle. Comment les propositions scientifiques - la réponse - ne seraient-elles pas inséparables de ces questions? Max Weber lui-même a oscillé entre diverses formules : la réponse étant à la fois compréhension et causalité, les propositions sont 
consacrées par cette dernière en leur validité hypothétiquement universelle. Mais cette formule supposerait que l'histoire ou les sciences sociales fussent tissées de relations causales, ce qui n'est pas le cas. La compréhension des calvinistes - quelle que soit l'action de ces derniers à l'origine du capitalisme - constitue la plus grande partie de l'étude elle-même. Ce n'est pas la causalité qui fonde la validité de l'interprétation que Max Weber propose du calvinisme.

En quoi peut consister la vérité de cette interprétation si celle-ci suppose un ensemble de concepts et que cet ensemble soit l'expression de l'interprète et de son temps ? Bien sûr, l'interprétation doit être compatible avec les faits on les documents mais, d'après Max Weber lui-même, plusieurs interprétations sont compatibles avec les données. Si l'on ne pousse pas l'analyse plus loin, l'épistémologie en reste à cette pluralité incohérente. Pour dépasser cette pluralité, il faut recourir à des analyses plus subtiles.

Max Weber insistait sur la notion de sens subjectif, c'est-à-dire du sens vécu par les acteurs historiques. Historiens et sociologues visent le sens vécu non le sens vrai. Mais le sens vécu est complexe : le charlatan se donne pour un vrai prophète, le démagogue pour un chef charismatique. L'expression que les acteurs donnent à leurs expériences, la conscience qu'ils ont ou qu'ils prétendent avoir [36] d'eux-mêmes ne constitue pas nécessairement la vérité historique. Si tous les sens sont interchangeables, si aucun n'a plus de portée que les autres, les sciences historiques et sociales ne peuvent échapper au chaos. En fait, la pluralité des sens est incontestable mais cette pluralité n'est pas quelconque.

Si le démagogue est un charlatan, l'historien se trompe qui voit en lui un chef charismatique. Si le prophète est authentique, l'historien se trompe qui voit en lui un mystificateur. Cette discrimination est un autre exemple de ces jugements de valeur internes aux univers que Max Weber ignorait dans son épistémologie explicite. Entre diverses interprétations d'un phénomène historique, une hiérarchie s'établit spontanément: le sens par rapport au milieu, le sens que lui donnent les disciples et le sens que lui donne le créateur lui-même ne se juxtaposent pas. Le sens d'une croyance religieuse ou d'un système philosophique est d'abord celui que le prophète (ou le théologien) et le philosophe lui ont donné. C'est par rapport à ce sens intrinsèque que se dé- 
tachent les autres. Et l'historien doit retrouver le sens premier avant de rechercher les autres.

Mais, dira-t-on, s'il applique ses concepts à l'interprétation de ce sens intrinsèque, il le renouvellera. A coup sûr. Mais la seule conclusion à tirer est que, au cas où il n'y aurait pas d'ordre interne à l'univers religieux ou philosophique, la suite des interprétations serait incohérente comme celle des œuvres. Proposition au reste évidente si l'on y réfléchit. Il n'y a pas d'histoire de l'humanité si l'humanité n'existe pas. Il n'y a pas d'histoire de la philosophie si la philosophie n'existe pas.

Comprenons bien la portée de ces affirmations. Il n'est pas nécessaire que l'humanité souscrive à une idée unique d'elle-même pour qu'elle ait une unité : il suffit que les diverses idées qu'elle a eues d'elle-même s'organisent d'un certaine manière, qu'elles ne paraissent pas sans, lien ou sans raison. De même, la philosophie acquiert l'unité d'une histoire dès lors que la ou les questions s'enchaînent, même si les réponses s'opposent.

La sociologie historique de Max Weber, de même, suppose que les institutions des diverses sociétés ne sont pas incomparables et que l'on puisse les ordonner en un système universellement valable de [37] concepts. M. Strauss reproche à Max Weber d'avoir méconnu possibilité et nécessité d'un système catégoriel universellement valable et, ayant admis la relativité historique de ce système, d'avoir faussé par le provincialisme sa sociologie de l'histoire universelle. La distinction des trois pouvoirs, traditionnel, rationnel et charismatique, écrit-il, n'est que l'expression d'une conjoncture singulière, celle qui suivit la Révolution française, dominée par l'antinomie de la tradition et de la raison, de l'ancien régime et de la révolution. Le troisième type de pouvoir, le pouvoir charismatique, est ajouté pour donner l'impression que le schéma est exhaustif.

Notre propos n'est pas de discuter ici des mérites et démérites de la distinction des trois pouvoirs. Mais - et sur ce point M. Strauss a raison contre l'épistémologie explicite de Max Weber - cette distinction est significative si et dans la mesure où les pouvoirs innombrables peuvent être subsumés sous trois types, autrement dit si et dans la mesure où la diversité historique n'est pas radicalement incohérente. Mais la non-incohérence n'implique pas encore - et sur ce point Max Weber 
a raison contre M. Strauss, à cette étape de l'argumentation du moins qu'il y ait un ordre supra-historique et un seul, dans lequel doivent être intégrées les singularités historiques. Il est vrai que la rivalité de l'ancien régime et de la révolution a été confondue à tort avec l'antinomie de la tradition et de la raison. Il est possible que l'antithèse traditionraison caractérise une période historique, et une seule. Mais le schéma wébérien n'en a pas moins, à mes yeux, une portée que lui refuse $\mathrm{M}$. Strauss.

Les trois termes - tradition, raison, charisme - correspondent à trois principes d'obéissance. L'homme obéit aux chefs que l'accoutumance consacre, que la raison désigne, que l'enthousiasme élève au-dessus des autres : les anciens, les organisateurs, les prophètes symbolisent ces trois sources de légitimité (que M. Strauss aurait pu admettre puisqu'il s'agit de modèles tout proches de ceux que connaît le citoyen non philosophe qui pense selon les notions de la vie quotidienne). De plus, l'autorité, que reçoit la fonction ou la compétence, que continue d'exercer le passé ou l'âge, est, à notre époque, en voie de rationalisation. Mais cette autorité rationnelle [38] ne suffit jamais : au niveau le plus élevé, le chef ne se réclame pas de la seule raison. En dernière analyse, il est transfiguré par une tradition, fût-ce celle de mécanismes rationnels, on par l'enthousiasme des foules. Le schéma wébérien aide à saisir le noyau du problème politique de notre civilisation.

Il est vrai que, posé en ces termes, le problème politique ne comporte pas de solution en soi la meilleure. On conçoit donc la possibilité d'une autre thématique en même temps que la légitimité partielle du schéma wébérien. Étant admis que la diversité historique n'est pas incohérente, l'opposition subsiste entre deux thèses : celle de Weber qui, excluant la recherche du régime le meilleur et de l'ordre universellement valable de la diversité, implique à la fois la légitimité d'une science comparative et le renouvellement historique de cette science, celle de M. Strauss qui lie la science comparative à l'ordre supra-historique dans lequel la diversité historique s'intègre.

3. De même que la radicale diversité des époques ou des institutions à travers le temps dévaloriserait la sociologie historique, de même la radicale irrationalité des décisions dévaloriserait le souci de la discrimination rigoureuse entre science et Politique, entre rap- 
ports aux valeurs et jugements de valeur. Après tout, pourquoi ne pas donner pour science ce qui est politique si, en dernière analyse, l'honnêteté n'est qu'un choix entre d'autres, ni plus ni moins valable que le choix contraire du cynisme, de l'hypocrisie ou de la confusion ? Le danger est d'autant plus réel qu'à certains moments Max Weber semble donner pour idéal, pour suprême aboutissement de son propre choix, le devoir pour chacun d'obéir à son dieu ou à son démon. Deviens ce que tu es, serait le dernier mot de ce que l'on ne devrait appeler ni morale ni sagesse mais peut-être « éthique personnelle».

Considéré par rapport à la tradition philosophique, Max Weber apparaît presque nietzschéen. Il se refuse à mettre les règles formelles de la moralité au-dessus de la relativité historique. Les impératifs kantiens ne sont pas moins caractéristiques d'une attitude entre d'autres que l'attachement aux dogmes chrétiens ou le culte des valeurs vitales. « Ne fais pas à [39] autrui ce que tu ne veux pas que l'on te fasse », ce commandement exige le refoulement de la volonté de puissance, la soumission aux principes d'égalité et de réciprocité entre individus. Or l'individu qui a choisi de s'accomplir à la manière de Calliclès refusera de s'incliner devant les interdits, promulgués par les faibles pour se protéger des forts, par les esclaves pour enchaîner les maîtres.

Que les règles formelles ne soient pas indépendantes d'une morale matérielle et que cette dernière soit incompatible avec le culte des valeurs vitales ou de la volonté de puissance, rien de plus incontestable. Mais si l'on met sur le même plan l'impératif de réciprocité et le rejet de cet impératif, alors le doute n'est pas permis : on tombe dans le nihilisme pur et simple. Tout est équivalent. «Tu ne tueras point» n'est, en dernière analyse, pas plus évident que « va jusqu'au bout de ton entreprise, fût-ce sur le corps de ta mère ". Si seule subsiste finalement l'obligation de fidélité à soi-même, le nietzschéen qui ne s'arrête devant rien vaut plus que le violent qu'arrêtent les scrupules.

Les implications nihilistes de quelques textes de Max Weber sont incontestables. J'ajoute que ce fut une des tendances de sa pensée. « Dieu est mort, tout est permis. » Ou, du moins, « Dieu est mort, chacun choisit son dieu, qui peut-être sera un démon ». Mais cette tendance n'est pas la seule qui traverse sa pensée. Le nihilisme nietzschéen auquel il aboutissait parfois était moins l'objet d'un choix résolu que la conséquence à demi involontaire d'un principe, à ses yeux 
fondamental : l'impossibilité de démontrer scientifiquement un jugement de valeur ou un impératif moral.

On peut lui accorder que la vérité des interdits, « ne fais pas à autrui ce que tu ne voudrais pas que l'on te fît », on « tu ne tueras point » n'est pas du même ordre que celle de la loi de gravitation ou des équations de la relativité. Ayant posé l'hétérogénéité entre la vérité universelle telle que la conçoit la science moderne de la nature, et tout le reste, Weber s'est ingénié à circonscrire dans les sciences de la « culture » un domaine où s'affirmerait une vérité, du môme type que celle de la physique, et il rejetait tout le reste dans les ténèbres extérieures.

[40]

L'erreur était double : la discrimination des questions (arbitraires) et des réponses (objectives) est plus malaisée que ses analyses ne le donneraient à penser. Si tout ce qui n'est pas vérité scientifique est arbitraire, la vérité scientifique elle-même serait l'objet d'une préférence, aussi peu fondée que la préférence contraire pour les mythes et les valeurs vitales.

Max Weber aurait pu sortir de ce cercle dans lequel il s'enfermait lui-même. En effet, s'il choisissait, pour employer son propre langage, la vérité scientifique, c'est que celle-ci était universelle, qu'elle était condition et foyer d'une communauté des esprits, à travers les frontières et les siècles. Le culte des valeurs vitales, l'affirmation de la volonté de puissance entraînent le refus de l'universalité : la rivalité, non la communauté, des individus serait l'essence de l'humanité. Même si l'on admet que logiquement la vérité de « 2 × $2=4 »$ n'est pas de même sorte que celle du « tu ne tueras point », il reste que le sens dernier de l'égalité arithmétique s'adresse à tous les hommes, universalité que l'interdiction de tuer retrouve d'une autre manière.

Les règles formelles de la morale rationaliste, d'origine chrétienne, dont la philosophie de Kant est la suprême expression, ne sont pas non plus affaire de goût comme les couleurs. Elles sont le développement logique de l'idée d'humanité, de société universelle des hommes, idée inséparable du sens profond de la vérité scientifique. Ces règles sont formelles parce que les institutions qui, de siècle en siècle, en sont l'adéquate réalisation, ne peuvent pas ne pas changer en fonction des techniques de la matière et de la société. 
4. Peu importe, aurait pu répondre Max Weber, que le philosophe établisse ou non des catégories multiples de propositions ou de commandements non scientifiques. Je n'ai pas été, je n'ai pas voulu être un philosophe. Je ne me suis aventuré sur le terrain de la philosophie que pour marquer les limites de la science et les antinomies de l'action. Or l'intention d'universalité qui anime la morale formelle ne se communique pas aux décisions des hommes d'action. en d'autres termes et pour parler un autre langage que Max Weber, la phénoménologie wébérienne de [41] l'action demeure valable, même si l'on soustrait la morale formelle à la relativité historique.

Reprenons, une à une, les données essentielles de cette phénoménologie. Y a-t-il deux morales, essentiellement distinctes, celle de la responsabilité et celle de la conviction ? Au premier abord, on est tenté, comme M. Leo Strauss, de répondre qu'aucun homme n'est moral qui agit exclusivement selon la morale de la conviction. Nul n'a le droit de se désintéresser des conséquences de ses actes. D'autre part, le souci des conséquences complète, sans les contredire, les mobiles de l'action. On agit par conviction et pour obtenir certains résultats.

Ces objections sont trop sommaires pour être convaincantes. Max Weber ne veut pas dire que le moraliste de la responsabilité n'a pas de conviction ni le moraliste de la conviction pas de sens de la responsabilité. Il suggère que, dans des situations extrêmes, les deux attitudes peuvent se contredire et qu'en dernière analyse l'un préfère l'affirmation intransigeante de ses convictions au succès et que l'autre sacrifie ses convictions aux nécessités de la réussite, l'un et l'autre étant moraux à l'intérieur d'une conception de la moralité. Romain Rolland est coupable au regard du moraliste de la responsabilité puisqu'il affaiblit la confiance des Français dans la justice de leur cause, il ne l'est pas au regard du moraliste de la conviction qui tient le respect de la vérité ou la fraternité des esprits pour des objectifs supérieurs au triomphe d'un pays, celui-ci fût-il porteur d'une cause relativement pure.

Cette réponse, à son tour, nous parait à la fois convaincante et sommaire. S'il est vrai que le moraliste de la conviction s'intéresse aux conséquences de ses actes, il n'est pas moins vrai que le moraliste de la responsabilité est souvent tenté de violer les règles formelles ou de sacrifier des valeurs concrètes pour atteindre des objectifs précis. Rien 
ne contribue plus à l'efficacité du combat que la bonne conscience des combattants. La mythologie ou, le mensonge contribuent plus souvent à forger cette bonne conscience que l'expression fidèle de la vérité. Max Weber aurait souscrit aux formules préférées de Julien Benda au moment de l'affaire Dreyfus : en tant que clerc, je défends la vérité, c'est-à-dire je proclame l'innocence de Dreyfus, mais qu'on ne [42] dise pas que je sers par là même ma patrie ou l'armée. Tout au contraire, en compromettant le prestige de l'état-major, je mets en péril l'autorité nécessaire des chefs militaires. Mais je suis comptable de la vérité, non de la puissance française.

Je ne pense pas que M. Leo Strauss nierait ces évidences. La question est de déterminer la place que l'on donne, dans la philosophie politique, aux antinomies inévitables de l'action. Toute une école, dont le plus illustre représentant est Machiavel, tient que l'essence de la politique se révèle précisément dans les situations extrêmes. Un politique doit être à la fois convaincu et responsable. Mais quand il faut mentir ou perdre, tuer ou être vaincu, quel choix est moral ? La vérité, répond le moraliste de la conviction, le succès, répond le moraliste de la responsabilité. Les deux choix sont moraux pourvu que le succès voulu par ce dernier soit celui de la Cité, non le sien propre.

L'antinomie me paraît essentielle, quand bien même, dans la majorité des cas, la prudence suggérerait un compromis raisonnable. La situation extrême dans laquelle le compromis devient difficile, sinon impossible, n'est pas exceptionnelle : le risque en surgit dès qu'il y a un conflit. Or Max Weber jugeait, non sans raison, que la politique est, par essence, conflit, entre les nations, entre les partis, entre les individus. Nul n'a jamais pensé que les règles formelles de la morale kantienne fussent en même temps les règles d'efficacité à l'usage des combattants de la bataille politique. Les exemples historiques, en euxmêmes discutables, de la morale de la conviction, donnés par Max Weber, n'ont pas été choisis par hasard: le pacifiste refuse d'entrer dans la guerre, le syndicaliste révolutionnaire - tel que le construit Weber - va jusqu'au bout de la volonté de guerre. L'un et l'autre rejettent toute prudence - ce que le moraliste de la responsabilité leur reproche à juste titre - mais l'un et l'autre peuvent répondre qu'ils ne veulent pas entrer l'un dans la guerre l'autre dans le compromis avec le régime abhorré. Le non inconditionnel, absolu, au risque de tout perdre, est l'expression dernière de ce que Max Weber appelait la morale de la 
conviction. Il n'y a pas de responsable qui ne sera acculé, un jour ou l'autre, à dire lui aussi "non », quel qu'en [43] soit le prix, weil er nicht anders kann, parce qu'il ne peut pas faire autrement. En bref, dans le monde humain tel qu'il est, la prudence dans laquelle se composent normalement affirmation de volonté et souci des conséquences, risque à chaque instant d'échouer devant l'obstacle de la contradiction entre morale universelle et nécessités de la lutte. La guerre est inséparable de la politique et l'homme de pensée qui entre dans la politique ne parvient ni à se soumettre entièrement aux obligations du combattant ni à s'en libérer totalement. Il rationalise ses propres contradictions dans l'antinomie des deux morales qui, au niveau de la phénoménologie de l'action politique, me paraît une conceptualisation fidèle de la conscience déchirée du "clerc dans la politique ».

« Sauver son âme ou sauver la Cité »: les chrétiens n'ont pas jugé, à travers l'histoire, que les deux termes fussent incompatibles. Nous ne voulons pas dire qu'ils le soient. Mais la politique est guerre et la morale universelle, celle du Christ ou celle de Kant, qui était restée, dans l'inconscient de Max Weber, la morale, est paix. Peut-être la sagesse enseigne-t-elle aux philosophes à ne pas entrer dans la guerre. S'ils y entrent, peut-être seront-ils prudents. Seront-ils véridiques ? Seront-ils purs?

5. Maints philosophes seront tentés de considérer l'antinomie des deux morales comme artificielle - deux tendances, complémentaires bien que divergentes parfois, étant présentées comme deux termes contradictoires. Au contraire, Max Weber voyait dans cette antinomie la marque de ce qui constitue authentiquement la condition humaine. Il retrouvait cette contradiction à trois niveaux, celui de la controverse politique, celui des fondements de l'ordre juste, celui des buts derniers de l'action ou de l'existence humaine.

a) Max Weber ne se lassait pas de montrer que toute mesure concrète - un droit de douane, une augmentation ou diminution d'impôt, une subvention - ne pouvait pas revêtir la dignité d'une vérité scientifique. Il est impossible d'avantager un groupe sans en désavan- 
tager un autre, impossible de démontrer qu'un progrès de la production globale n'est pas payé trop cher par la ruine de petits [44] commerçants ou le déclin d'une région défavorisée, impossible de prouver que les suites, en elles-mêmes déplorables, d'une mesure fiscale sont compensées par les bénéfices qu'on en attend. Cette sorte d'argumentation est à la fois indiscutable et sans grande .portée. Elle renvoie à la problématique, désormais classique, de l'intérêt collectif et des intérêts individuels.

Mettons-nous à l'intérieur de l'univers de l'économie moderne. Une mesure ne peut être dite avec certitude conforme à l'intérêt commun que dans les circonstances où elle accroît les satisfactions de quelques-uns sans réduire les satisfactions de personne (les satisfactions étant mesurées par l'expression objective qu'en constituent les revenus disponibles). Même dans cette hypothèse suggérée par la définition que donne Pareto du maximum d'intérêt Pour une collectivité, il subsiste, en rigueur, une incertitude : une mesure qui accroîtrait les revenus de quelques-uns sans réduire ceux de personne pourrait aggraver l'insatisfaction créée par l'injustice au ras où la progression d'ensemble accentuerait l'inégalité de la répartition. Lors même que la mesure favorable aux uns ne l'est pas aux autres, il n'en résulte pas que le choix, qui effectivement n'est pas rigoureusement scientifique, soit arbitraire et que, faute de démonstration universellement valable, il ne subsiste que la lutte sans terme et sans règlement équitable. À l'intérieur de la société industrielle, la controverse est permanente, elle n'est pas une lutte à mort, elle n'exclut pas le débat raisonnable et la solution négociée.

Le but est admis : l'augmentation du produit national et l'atténuation des inégalités de répartition. Ces deux objectifs de la croissance et de la réduction des inégalités ne suggèrent pas toujours les mêmes décisions. À trop insister sur l'égalité, on risque de compromettre la croissance. L'obsession de la croissance conduit à méconnaître les souffrances des hommes et à sacrifier les vivants aux générations à venir. Il n'est pas de mesure dite technique - taux d'intérêt, droit de douane, impôt - qui n'ait des implications politiques et sociales, autrement dit qui n'affecte la répartition des revenus et du pouvoir en même temps que le développement d'ensemble. Les hypothèses sur lesquelles [45] se fonde la doctrine du Welfare (par exemple qu'une répartition égalitaire d'un certain volume de revenus augmente les satis- 
factions, le revenu transféré d'un riche à un pauvre augmentant plus la satisfaction de ce dernier qu'il ne diminue celle du premier) sont toutes discutables, en tout cas non scientifiques. Mais on aurait tort de poser l'alternative de la science et de la décision arbitraire. Entre la proposition rationnelle, valable pour tous parce que démontrée selon des méthodes qui s'imposent à tous, et le choix que chacun fait seul et qui .n'oblige personne d'autre, il y a place pour la décision raisonnable, la décision fondée en raison, bien que contraire à l'intérêt de quelques-uns.

Discussion et choix raisonnables se situent à l'intérieur de la civilisation industrielle et même à l'intérieur d'un régime déterminé. Qui demeure hostile à l'industrialisation ou qui refuse inconditionnellement la propriété privée des instruments de production n'entre pas dans cette discussion raisonnable : il est dans l'histoire et celle-ci est faite d'une lutte inexpiable entre les hommes, les partis et les dieux.

b) Max Weber jugeait ensuite irréductibles les conflits dont l'enjeu est la définition de l'ordre juste. Quelles sont les exigences du concept d'égalité ? Telle est, au fond, l'interrogation dernière à quoi se ramène le débat sur l'ordre juste. Or, disait Max Weber, deux réponses sont également valables : ou bien on doit davantage à celui qui accomplit davantage, ou bien on lui demande davantage. Faut-il favoriser l'élite et l'aider à s'épanouir ? Ou bien, au contraire, la législation doit-elle agir en sens contraire de la nature, rétablir sans cesse l'égalité que la nature tend, avec une égale constance, à détruire ? M. Strauss juge, non sans motif, que Max Weber n'a pas formulé l'antinomie avec suffisamment de rigueur, ni moins encore formulé la thèse de l'« égalité à tout prix » en termes tels qu'elle paraisse aussi plausible que la thèse opposée des inégalités naturelles que toute société doit accepter.

Nous ne pourrions, sans dépasser le cadre de cette introduction, discuter à fond le problème de l'ordre juste. Quelques remarques suffiront pour dégager ce qui nous paraît indiscutable dans la [46] conception wébérienne des antinomies de l'égalité et valable dans le refus qu'oppose M. Strauss à cette transfiguration tragique des antinomies.

Considérons les doctrinaires de la politique aussi bien que les chefs de partis. Le fait est que les uns sont plus soucieux de laisser libre carrière aux talents les autres d'empêcher les différences de conditions de 
s'accuser. Que l'on relise Alain : le philosophe du radicalisme reconnaît avec Auguste Comte qu'en toute société il y a des riches et des puissants, mais il se préoccupe plus de prévenir les abus de la richesse et de la puissance que d'accorder aux plus productifs la récompense méritée par leurs capacités. D'autres écrivains s'orientent dans l'autre direction et se demandent comment assurer la sélection d'une élite et renforcer l'autorité des meilleurs. Qu'il s'agisse de revenus ou de pouvoir, il ne me parait pas douteux qu'au niveau des idéologies économico-sociales, deux tendances se dessinent, l'une qui veut donner à tous des conditions de vie aussi semblables que possible, l'autre qui veut élargir la prime due aux capacités, l'une qui souhaite empêcher les gouvernants, fussent-ils les meilleurs, d'exercer un pouvoir illimité, l'autre qui se donne pour objectif de consolider le règne des gouvernants dignes de leurs fonctions.

Ces préférences divergentes ne peuvent pas ne pas intervenir chaque fois qu'il faut résoudre un problème déterminé. Qu'il s'agisse de fiscalité, de système d'éducation, de propriété des instruments de production, le doctrinaire de l'égalité penchera dans un sens, le doctrinaire de la hiérarchie naturelle et sociale dans un autre. En ce sens Max Weber n'avait pas tort de marquer l'hétérogénéité de la décision politique et de la démonstration scientifique. Mais avait-il raison d'assimiler des préférences divergentes à une contradiction fondamentale, irréductible?

Cette assimilation était obtenue par les postulats suivants - $1^{\circ}$ les hommes sont naturellement inégaux mais cette inégalité naturelle étant l'injustice suprême en même temps qu'originelle, le théoricien ou le praticien de la politique est en droit de penser qu'il faut l'effacer et d'agir en vue d'atteindre cet objectif ; $2^{\circ}$ la société a besoin des mieux doués, dans l'ordre de l'esprit ou de la moralité, mais celui [47] qu'anime la passion d'égalité est en droit d'oublier les considérations pragmatiques pour n'obéir qu'au seul impératif de la justice telle qu'il l'interprète ; $3^{\circ}$ quand des considérations diverses, et pour une part divergentes, s'imposent simultanément, celui qui méconnaît l'une d'elles ne vaut pas moins que celui qui s'efforce de les retenir toutes ensemble, en d'autres termes l'extrémiste est au niveau du modéré, le «mono-idéiste » n'est pas inférieur au sage.

Il suffit de formuler ces postulats pour voir qu'ils sont pour le moins discutables et qu'ils ont tous la même origine, psychologique 
plus encore que logique : en dehors de la science, il n'y aurait que choix et, puisque ce choix est par essence non scientifique, celui qui va jusqu'au bout de sa foi ne saurait être condamné quand bien même il serait entraîné par elle jusqu'au fanatisme. Il suffit d'accepter la pluralité des considérations (on, si l'on préfère, des valeurs), sans postuler une irréductible antinomie entre celles-ci, pour apercevoir l'issue. Souci de l'égale dignité de tous les hommes et, dans nos sociétés, de la réduction des inégalités économiques, acceptation des inégalités naturelles et nécessité de favoriser l'épanouissement des talents, reconnaissance de la hiérarchie sociale et volonté de la rendre équitable par le choix des chefs et tolérable aux gouvernés par la limitation des prérogatives des puissants, quiconque veut ignorer l'une ou l'autre de ces données ne commet peut-être pas une erreur scientifique ou une faute morale mais il est déraisonnable. Peut-être Max Weber se bornerait-il à répondre : certes, mais pourquoi vaut-il mieux être raisonnable que passionné? peut-être les critères de l'ordre juste sont-ils multiples plutôt que contradictoires, peut-être les oppositions apparaissent-elles sur le plan des solutions concrètes, historiques, et non sur celui des principes qui, au niveau le plus élevé d'abstraction, sont complémentaires et non contradictoires. Il reste que les exigences de chaque personne, de chaque civilisation, de chaque époque sont incomparables. Chaque existence a son Dieu et les dieux sont en lutte.

c) Venons à la troisième contradiction, la plus profonde, décisive si elle est authentique, celle des valeurs. La contradiction des valeurs, Max Weber [48] la suggérait pour ainsi dire comme si elle allait de soi, il ne la démontrait pas et les exemples qu'il en donne ne sont pas tous convaincants. Une chose, dit-il, peut être belle précisément parce qu'elle n'est pas bonne, ainsi les Fleurs du Mal. Certes, l'œuvre de Baudelaire n'est morale ni en son objet ni même peut-être en l'intention du créateur, à supposer que l'intention du poète puisse être jugée par rapport à la morale. Mais la beauté des Fleurs $d u$ Mal n'a pas pour cause l'immoralité des thèmes ou l'immoralité (supposée) du créateur. Entre le sens spécifique de l'œuvre d'art et la finalité de la conduite qui se veut morale, entre la beauté et le devoir, il n'y a pas lutte inexpiable mais une simple pluralité comparable à celle des castes de l'Inde.

Nulle démonstration, dit-il encore, ne permet de trancher la question de la valeur respective de la culture allemande et de la culture 
française. Certes, mais la question est-elle significative ? À la rigueur, on établit une hiérarchie entre les œuvres à l'intérieur d'un univers donné, on ne saurait établir une hiérarchie entre des ensembles historiques, dont chacun contribue à la richesse de l'histoire totale, dont chacun apporte quelque chose que l'autre ne possède pas.

Ces remarques sont à ce point évidentes que l'on ne parvient pas à se convaincre que Max Weber les ait méconnues.

Reprenons donc la phrase la plus caractéristique

« La sagesse populaire nous enseigne qu'une chose peut être vraie bien qu'elle ne soit ni belle, ni sainte, ni bonne et parce qu'elle ne l'est pas. » Pourquoi Max Weber a-t-il conclu de cette sagesse populaire non pas que les univers spirituels avaient chacun leur loi propre niais que le polythéisme grec évoquait à juste titre une lutte des dieux?

L'homme ne peut pas se conduire à la fois selon les exigences de la sainteté et selon les exigences de la moralité temporelle. 'rendre l'autre joue, c'est manque de dignité si ce n'est sainteté. D'autre part, s'il est vrai qu'Apollon et Mars, Vénus et Minerve ne sont pas condamnés à se combattre, chaque personne, individuelle ou collective, ne peut pas sacrifier simultanément à tous les dieux. Le philosophe peut bien concevoir la diversité des accomplissements humains comme un enrichissement, [49] l'homme, seul entre d'autres, ne peut choisir un accomplissement sans du même coup renoncer à d'autres. Une société ne peut exceller à la fois en tous les ordres de l'action, de la sainteté, de l'art et de la méditation. En ce sens, toute existence est choix et celuici comporte plus de «non » que de « ouf», il condamne chaque personne à se compter plus d'ennemis que de fidèles.

Il est évident que l'individu ne réalise jamais que certaines des potentialités de son être ou de l'humanité. Il est encore évident qu'une époque est prisonnière de l'idée qu'elle se fait de la beauté. Mais pas plus que l'individu ne choisit contre l'éthique du soldat en choisissant le métier de savant, Phidias ne choisit la statuaire grecque contre celle de l'Elephanta. La particularité de chaque accomplissement historique n'entraîne pas le conflit entre ces particularités. Tout au plus créeraitelle un chaos spirituel si la relativité pure et simple était le dernier mot de la réflexion sur l'histoire. 
La guerre entre les dieux ou du moins entre les fidèles des dieux surgit si le culte dû à un dieu contredit directement le culte dû à un autre. Dans la cité, le citoyen ou l'homme d'action doit prendre position pour un parti ou pour une cause, contre d'autres partis et d'autres causes, tous les partis se réclamant des valeurs suprêmes. À l'époque où les chrétiens combattaient le panthéon romain, les acteurs de l'histoire croyaient à des dieux, voués à une lutte à mort. Il en allait de même (à un moindre degré) quand les révolutionnaires français abattaient les trônes et les autels. Il en va encore de même quand les communistes dissolvent les parlements et nationalisent les instruments de production. Quel que soit le jugement du philosophe, l'existence historique est faite de combats douteux où nulle cause n'est pure, nulle décision sans risque, nulle action sans conséquences imprévisibles. Peutêtre le philosophe discerne-t-il au-delà de ce tumulte la fraternité des dieux, l'historien constate la fureur fratricide des Églises.

La guerre des Églises n'est pas la guerre des dieux, mais la phrase de Max Weber nous suggère le passage de l'une à l'autre : "Tendre l'autre joue, c'est manque de dignité si ce n'est sainteté ». Le [50] même acte, selon le sens que l'on donne à sa vie, est bon ou mauvais.

Si Max Weber choisit pour exemple de la contradiction l'attitude à l'égard de l'ennemi, le motif de ce choix, apparemment accidentel, n'est pas à chercher loin. Morale de Kant on éthique du Sermon sur la montagne s'appliquent aux hommes de paix et de bonne volonté. Sur cette terre, les hommes sont en guerre dans la cité, les cités sont en guerre sur la scène du monde. Chacune des cités défend une cause en elle-même valable (comment établir une hiérarchie entre les nations, entre la culture allemande et la culture française ?). Les partis, dont les moyens on les objectifs immédiats sont le moins justifiables, peuvent apporter à terme des bienfaits dont se féliciteront nos arrière-neveux. Une fois de plus, Max Weber n'aperçoit qu'une seule issue pour qui ne se soumet pas aux impératifs du combat: l'indifférence aux conséquences, qui fonde la morale de la conviction ou la sainteté de la nonrésistance individuelle par fidélité à certains enseignements du Christ.

Le saint, le héros ne peuvent agir de même en une même conjoncture. La religion commande-t-elle de «tendre l'autre joue » si cette formule équivaut à recommander la non-résistance au mal ? Toutes les sociétés ont, d'une manière ou d'une autre, reconnu la pluralité que Max Weber voulait belliqueuse et pathétique. Le guerrier ne comprend 
pas toujours le brahmane, mais le brahmane n'ignore jamais le guerrier : il hésite entre l'utopie d'une cité où le guerrier obéirait à ses ordres et la sagesse qui se satisfait d'une cité où les philosophes auront le loisir de penser mais non l'ambition de régner, résignés à « l'histoire pleine de bruit et de fureur et qui ne signifie rien ». Quel que soit son choix, il n'apercevra pas de "guerre des dieux ». Si le philosophe adhère à l'utopie, il conserve l'espoir de la réconciliation. S'il est sage, donc résigné à la non-sagesse des autres, pourquoi verrait-il un conflit inexpiable entre lui et les insensés, entre ceux qui méditent et ceux qui combattent ? Le héros n'ignore ni ne méprise le saint : il méprise celui qui tend l'autre joue par lâcheté, non celui qui tend l'autre joue par un courage supérieur.

Pourquoi Max Weber est-il à ce point assuré que [51] les conflits de l'Olympe sont inexpiables ? À la fois parce que les conflits étaient en lui et parce que ces conflits sont l'objet privilégié de l'étude sociologique. Le rationaliste reconnaît la lutte de la foi et de l'incroyance, il admet que ni l'une ni l'autre ne sont démontrables scientifiquement. Souscrivant à la vérité de l'incroyance, il conclut non à la guerre des dieux, mais à la diffusion progressive des Lumières ou bien à la persistance des illusions. Aux yeux du croyant, en revanche, c'est la foi qui fixe le sens du scepticisme. La formule de la "guerre des dieux » est la transposition d'un fait indiscutable - les hommes se sont fait des représentations incompatibles du monde - en une philosophie que personne ne vit ni ne pense parce qu'elle est contradictoire (toutes les représentations sont équivalentes, aucune n'étant ni vraie ni fausse).

Max Weber pouvait avoir le sentiment de vivre cette philosophie. Non croyant, il avait gardé la nostalgie de la foi et il était convaincu qu'avec la religion se perdaient des valeurs spirituelles irremplaçables. Kantien, il était passionné d'action politique et il voyait une antinomie irréductible entre les règles de la morale formelle et les exigences de l'action, c'est-à-dire de la lutte. Sociologue, il constatait que les civilisations, les peuples, les partis pensent et agissent selon des systèmes de valeurs et d'interprétations au moins divergents, sinon opposés. Le déchirement de l'incroyance, l'antinomie de la moralité et de la politique, la diversité des cultures devenaient, sous sa plume, autant de preuves de la "guerre des dieux ». Des analyses phénoménologiques, en elles-mêmes vraies, s'exprimaient en une philosophie humainement impensable. 
La méthodologie de Max Weber, comme l'a dit après beaucoup d'autres M. Leo Strauss, est inséparable d'une philosophie. Mais de cette remarque indiscutable, il tire probablement des conclusions inverses de celles que j'en tirerais moi-même. Il suggère que la méthodologie de Max weber aurait été faussée par sa philosophie. J'y consens sur certains points : le langage néo-kantien (distinction des faits et des valeurs, rapports aux valeurs et [52] jugements de Valeur) a compromis l'élaboration d'une théorie de la compréhension, il lui a interdit d'admettre des appréciations liées à la compréhension ellemême, dans le cas des œuvres dont le sens né se sépare pas de la qualité. Mais, pour l'essentiel, ce n'est pas la méthodologie qui a été victime de la philosophie, c'est la méthodologie qui a inspiré à tort une philosophie. Les limites de la science, les antinomies de la pensée et de l'action sont les apports authentiques d'une description phénoménologique de la condition humaine. La philosophie du déchirement, si l'on peut dire, transpose ces données en un autre langage et leur donne un sens différent. Cette transposition suppose le refus de discrimination entre valeurs vitales et accomplissement raisonnable, l'irrationalité totale du choix entre les partis politiques ou les représentations du monde en lutte, l'équivalence morale et spirituelle entre les attitudes, celle du sage et celle de l'insensé, celle du fanatique et celle du modéré.

Parce que Max Weber avait gardé la nostalgie de la foi qu'il avait perdue, il jugeait, en dernière analyse, injustifiable la science à laquelle il consacrait sa vie. La décision lui paraissait d'autant plus humaine qu'elle était plus libre. Mais il ne se demandait pas si une telle décision pouvait être sans raisons et si ces raisons ne renvoyaient pas inévitablement à des principes universels. La diversité historique des valeurs, des croyances et des cultures est le fait : l'historien et le sociologue ne peuvent pas ne pas constater ce fait premier. Mais ils ne pourraient le donner pour dernier sans rendre impossible la science de cette diversité. L'ordre de cette diversité permet-il d'établir la fin unique de l'aventure, la destination naturelle de l'homme ou des socié- 
tés? Nous n'avons pas, dans les pages précédentes, prétendu répondre à la question.

Les choix auxquels est effectivement condamné l'homme historique, parce que la science est limitée, l'avenir imprévisible et que les valeurs sont, à courte échéance, contradictoires, ne sont pas démontrables. Mais la nécessité de choix historiques n'implique pas que la pensée soit suspendue à des décisions essentiellement irrationnelles et que l'existence s'accomplisse dans une liberté qui refuserait de se soumettre même à la Vérité. 


\section{LE MÉTIER ET LA VOCATION DE SAVANT}

\section{9}

Wissenschaft als Beruf

Vous m'avez demandé de vous parier du métier de savant. Or nous autres économistes, nous avons l'habitude d'une certaine pédanterie, à laquelle je voudrais rester fidèle : nous commençons toujours par examiner les conditions extérieures d'un problème ; je poserai donc ici la question : comment se présente le métier de savant, au sens concret du mot? Pratiquement cela revient à dire aujourd'hui : comment se présente la, situation d'un étudiant qui a terminé ses études et qui est décidé à faire de la science son métier, dans le cadre de la vie universitaire ? Pour comprendre combien particulière est sur ce point la situation allemande, il est utile de procéder par comparaison et de rappeler comment les choses se passent à l'étranger, là où le régime accuse les différences les plus nettes avec le nôtre sous ce rapport, je veux dire aux États-Unis. 
Chez nous - chacun le sait - le jeune homme qui se consacre à la science commence normalement sa carrière par le poste de Privatdozent. Après avoir conféré avec le spécialiste de la discipline qu'il a choisie et après avoir obtenu son consentement, il se fait habiliter pour l'enseignement supérieur en présentant un ouvrage et en se soumettant aux épreuves d'un examen, le plus souvent formel, devant le jury de la faculté [54] d'une université. Désormais il pourra donner des cours en choisissant lui-même son sujet dans le cadre de sa venia legendi. Mais il ne perçoit aucun traitement, il n'a d'autre rémunération que la contribution des étudiants [Kolleggeld]. En Amérique on débute d'une tout autre façon dans la carrière : on y est d'abord engagé comme « assistant ». D'une manière assez voisine, par exemple, de celle qui a ordinairement cours dans nos grands Instituts des Facultés des sciences et de médecine où l'habilitation formelle comme Privatdozent n'est convoitée que par une fraction des assistants et souvent tardivement. Cette différence avec le système américain signifie pratiquement que chez nous la carrière d'un homme de science est édifiée somme toute sur des bases ploutocratiques. En effet, il est extrêmement risqué pour un jeune savant sans fortune personnelle d'affronter les aléas de la carrière universitaire. Il doit pouvoir subsister par ses propres moyens, du moins pendant un certain nombre d'années, sans être aucunement assuré d'avoir un jour la chance d'occuper un poste qui pourra le faire vivre décemment. Aux États-Unis par contre règne le système bureaucratique. Dès son entrée dans la carrière, le débutant touche un traitement, il est vrai médiocre, puisque la plupart du temps il correspond à peine au salaire d'un ouvrier non qualifié. Néanmoins il débute en occupant une situation apparemment stable, étant donné qu'il touche un traitement fixe. Il est toutefois de règle qu'on peut lui donner congé, comme à nos assistants en Allemagne, s'il ne répond pas aux espoirs mis en lui ; il doit même s'y attendre assez souvent, sans compter sur aucun ménagement. Que signifient ces « espoirs » ? Tout simplement qu'il fasse « salle pleine ». Pareille mésaventure ne menace point le Privatdozent. Une fois en place, on ne peut l'en déloger. Certes, il ne formule aucune revendication. Mais il pense pourtant, chose humainement [55] compréhensible, qu'il possède une sorte de droit moral à des ménagements lorsqu'il a exercé des années durant. On tient également compte de cette 
situation acquise - et cela est souvent important - au moment de l'« habilitation» éventuelle d'autres Privatdozenten. D'où le problème : faut-il en principe accorder l'« habilitation » à tout jeune savant ayant donné les preuves de ses capacités ou bien faut-il prendre en considération les « besoins de l'enseignement» et donner ainsi aux Dozenten en place le monopole de l'enseignement ? Cette question pose un pénible dilemme qui est lié au double aspect de la vocation universitaire dont nous parlerons tout à l'heure. La plupart du temps on se décidé en faveur de la seconde solution. Mais elle ne fait qu'augmenter certains dangers. En effet, en dépit de la plus grande probité personnelle, le professeur titulaire spécialiste de la discipline en question est malgré tout tenté de donner la préférence à ses propres élèves. Si je me réfère à mon expérience, j'avais adopté le principe suivant : je demandais à l'étudiant qui avait fait sa thèse sous ma direction d'aller se faire agréer et « habiliter» par un professeur d'une autre université. Il en est résulté qu'un de mes élèves, et des plus méritants, a été éconduit par mes collègues parce qu'aucun n'ajoutait foi aux motifs allégués.

Il existe une autre différence avec le régime américain. En Allemagne le Privatdozent a en général moins de cours à faire qu'il ne le désire. En droit, il peut certes faire tous les cours de sa spécialité. Mais une pareille façon de faire est considérée comme une indélicatesse inouïe à l'égard des Dozenten plus anciens ; en conséquence les " grands » cours sont réservés au professeur et le Dozent se contente des cours secondaires. Il y trouve l'avantage, peut-être involontaire, de pouvoir disposer pendant sa jeunesse de loisirs qu'il peut consacrer au travail scientifique.

[56]

En Amérique, l'organisation est fondamentalement différente. C'est précisément pendant ses jeunes années que l'assistant est littéralement surchargé de travail, justement parce qu'il est rémunéré. Dans le département d'études germaniques par exemple, le professeur titulaire fait environ trois heures de cours sur Gœthe, et c'est tout - alors que le jeune assistant s'estime heureux si, au cours de ses douze heures hebdomadaires, on l'autorise à faire, à côté des travaux pratiques d'allemand, quelques leçons sur des écrivains d'un plus grand mérite que, disons, Uhland. En effet, ce sont les instances officielles 
de sa spécialité qui fixent le programme et l'assistant doit s'y plier tout comme chez lions l'assistant d'un Institut.

Or nous pouvons observer clairement que, dans de nombreux domaines de la science, les développements récents du système universitaire allemand s'orientent dans la direction du système américain. Les grands instituts de science et de médecine sont devenus des entreprises du a capitalisme d'État ». Il n'est plus possible de les gérer sans le secours de moyens considérables. Et l'on voit apparaître, comme partout ailleurs où s'implante une entreprise capitaliste, le phénomène spécifique du capitalisme qui aboutit à " couper le travailleur des moyens de production ». Le travailleur - l'assistant - n'a d'autre ressources que les outils de travail que l'État met à sa disposition; par suite il dépend du directeur de l'institut de la même façon qu'un employé d'une usine dépend de son patron - car le directeur d'un institut s'imagine avec une entière bonne foi que celui-ci est son institut : il le dirige clone à sa guise. Aussi la position de l'assistant y est-elle fréquemment tout aussi précaire que celle de toute autre existence " prolétaroïde » ou celle de l'assistant des universités américaines.

[57]

Comme les autres secteurs de notre vie, l'université allemande s'américanise sur d'importants chapitres. Je suis convaincu que cette évolution touchera même des disciplines dans lesquelles le travailleur est personnellement propriétaire de ses moyens de travail (essentiellement de sa bibliothèque). Pour le moment le travailleur de ma spécialité est encore dans une large mesure son propre maître, à l'instar de l'artisan d'autrefois dans le cadre de son métier. Mais l'évolution se fait à grands pas.

On ne peut nier les avantages techniques incontestables de cette évolution comme dans n'importe quelle autre entreprise ayant à la fois les caractères capitaliste et bureaucratique. Mais le nouvel « esprit » est bien différent de la vieille atmosphère historique des universités allemandes. Il y a un abîme, extérieurement et intérieurement, entre le chef de cette sorte de grande entreprise universitaire capitaliste et l'habituel professeur titulaire du vieux style. Cela se traduit même dans le comportement intime. Mais je ne veux pas entrer dans les détails. L'ancienne constitution universitaire est devenue fictive 
aussi bien dans son esprit que dans sa structure. Néanmoins, il est un aspect propre à la carrière universitaire qui n'a pas disparu et qui se manifeste même d'une façon encore plus sensible : le rôle du hasard. C'est à lui que le Privatdozent et surtout l'assistant doivent de parvenir éventuellement un jour à occuper un poste de professeur titulaire à part entière on surtout celui de directeur d'un institut. Bien sûr, l'arbitraire ne règne pas seul dans ce domaine, mais il commande pourtant dans une mesure inhabituelle. Je ne connais guère de carrière au monde où il joue un si grand rôle. Je puis en parler d'autant mieux que personnellement je dois à un concours de circonstances particulièrement heureuses d'avoir été appelé très jeune à occuper une chaire de professeur titulaire dans une spécialité où des camarades de mon âge avaient indubitablement [58] produit déjà beaucoup plus que moi-même. Fort de cette expérience, je crois posséder un oeil plus pénétrant pour comprendre le sort immérité de nombreux collègues à qui la fortune n'a pas souri et ne sourit pas encore et qui, avec ces procédés de sélection, n'ont jamais pu occuper, malgré tout leur talent, le poste qu'ils méritaient.

Si le hasard et non la seule valeur joue un si grand rôle, la faute n'en incombe pas uniquement ni même surtout aux faiblesses humaines qui interviennent évidemment dans cette sélection comme dans toute autre. Il serait injuste d'imputer aux petits personnages des facultés ou des ministères la responsabilité d'une situation qui fait qu'un si grand nombre de médiocres jouent incontestablement un rôle considérable dans les universités. Il faut plutôt en chercher la raison dans les lois mêmes de l'action concertée des hommes, surtout dans celle de plusieurs organismes, en l'espèce dans la collaboration entre les facultés qui proposent les candidats et le ministère qui les nomme. Nous pouvons en trouver un parallèle dans l'élection des papes qui nous a fourni depuis de nombreux siècles l'exemple manifeste le plus important de ce type de sélection. Le cardinal que l'on indiquait comme "favori » avait très rarement la chance d'être élu. En règle générale elle comblait le candidat numéro deux ou trois. On constate le même phénomène lors des élections présidentielles aux États-Unis. Ce n'est qu'exceptionnellement que le candidat numéro un et aussi le plus marqué est « désigné » par les conventions nationales des partis : la plupart du temps on choisit le candidat numéro deux et souvent le candidat numéro trois. Les Américains ont déjà même créé des ex- 
pressions techniques et sociologiques pour caractériser ces catégories de candidats. Il serait évidemment intéressant d'examiner d'après ces exemples les lois d'une sélection opérée par acte de volonté collective, mais ce n'est pas notre [59] affaire aujourd'hui. Ces mêmes lois s'appliquent aussi aux élections dans les assemblées universitaires. Ce dont il faut s'étonner, ce n'est pas que des méprises arrivent souvent dans ces conditions ; mais plutôt que, toutes proportions gardées, l'on y constate malgré tout un nombre aussi considérable de nominations justifiées. Ce n'est que dans les quelques pays où le Parlement a son mot à dire ou encore dans ceux où les monarques interviennent pour des raisons politiques (le résultat est le même dans les deux cas), ainsi que cela arrivait fréquemment en Allemagne jusqu'à ces derniers temps et à. nouveau de nos jours avec les détenteurs du pouvoir révolutionnaire, que l'on peut être certain que les médiocres et les arrivistes ont seuls une chance d'être nommés.

Aucun professeur d'université n'aime se rappeler les discussions qui eurent lieu lors de sa nomination, car elles sont rarement agréables. Je puis pourtant dire que parmi les nombreux cas qui me sont connus j'ai constaté sans exception l'existence d'une bonne volonté soucieuse de ne faire intervenir dans la décision que des raisons purement objectives.

Il faut en outre comprendre clairement que les défaillances dans la sélection opérée par la volonté collective n'expliquent pas à elles seules le fait que la décision concernant les destinées universitaires est livrée dans une grande mesure au " hasard». Tout jeune homme qui croit avoir la vocation de savant doit se rendre compte que la tâche qui l'attend présente un double visage. Il doit posséder non seulement les qualifications du savant, mais aussi celle du professeur. Or, ces ceux aspects ne coïncident absolument pas. L'on peut être un savant tout à fait éminent et en même temps un professeur terriblement médiocre. Je songe à l'activité professorale d'hommes tels que Helmholtz ou Ranke qui ne constituent certainement pas des exceptions. En vérité [60] les choses se présentent de la façon suivante nos universités, particulièrement les petites universités, se font entre elles la concurrence la plus ridicule pour attirer les étudiants. Les locataires de chambres d'étudiants, bornés comme des paysans, organisent des fêtes en l'honneur du millième étudiant, et de préférence une retraite aux flambeaux pour saluer le deux millième. Le revenu que constitue la contribution 
des étudiants est, il faut bien l'avouer, conditionné par le fait que d'autres professeurs qui «attirent un grand public d'étudiants » occupent les chaires dans les spécialités voisines. Même en faisant abstraction de cette circonstance, il reste vrai que le nombre des auditeurs est un critère numérique tangible de la valeur, alors que la qualité du savant est du domaine de l'impondérable. Il arrive fréquemment (ce qui est tout à fait naturel) qu'on conteste justement celle-ci aux novateurs audacieux. C'est pourquoi tout est presque toujours subordonné à l'obsession de la salle pleine et des bienfaits qu'elle apporte. Lorsqu'on dit d'un Dozent qu'il est un mauvais professeur, cela revient la plupart du temps à prononcer une sentence de mort universitaire, fût-il le tout premier savant du monde. On tranche donc la question des bons et des mauvais professeurs par l'assiduité dont Messieurs les étudiants veulent bien les honorer. Or c'est un fait que les étudiants font affluence chez un professeur pour des raisons qui sont dans une très large mesure -si large même qu'on a peine à y croire - étrangères à la science comme le tempérament ou l'inflexion de la voix. Une expérience personnelle, déjà suffisamment riche, et une réflexion exempte de toute illusion, m'ont conduit à me méfier fortement des cours suivis par une masse d'étudiants, bien que sans doute pareille aventure soit inévitable. Mais il faut mettre la démocratie là où elle convient. En effet l'éducation scientifique telle que nous devons la donner par tradition dans les universités [61] allemandes est une affaire d'aristocratie spirituelle. Il est vain de vouloir se le dissimuler. Or il est également vrai d'autre part que, de toutes les tâches pédagogiques, la plus difficile consiste à exposer les problèmes scientifiques de telle manière qu'un esprit non préparé mais doué puisse les comprendre et se faire une opinion propre - cela constitue pour nous le seul succès décisif. Personne ne le contestera, mais ce n'est point le nombre des auditeurs qui décide de la solution de ce problème. Et - pour revenir à notre sujet - cet art dépend d'un don personnel et ne se confond absolument pas avec les qualités scientifiques d'un savant. Contrairement à la France, nous n'avons pas de compagnie d'Immortels de la science, mais ce sont les universités qui 'doivent par tradition répondre aux deux exigences de la recherche et de l'enseignement. C'est pur hasard si cette double aptitude se rencontre en un seul homme.

La vie universitaire est donc livrée à un hasard aveugle. Lorsque de jeunes savants viennent nous demander conseil en vue de leur ha- 
bilitation, il nous est presque impossible de prendre la responsabilité de notre approbation. S'il s'agit d'un juif on lui dit naturellement : lasciate ogni speranza. Mais il faut aussi en âme et conscience poser aux autres candidats la question: " Vous croyez-vous capables de supporter sans dommage ni amertume que, d'année en année, on vous préfère des médiocres après d'autres médiocres? » Évidemment on obtient chaque fois la même réponse : «Bien sûr ! je ne vis que pour ma vocation. » Cependant je n'ai connu, personnellement du moins, que très peu de candidats qui aient supporté cette situation sans dommage pour leur vie intérieure.

Voilà ce qu'il était nécessaire de dire des conditions extérieures du métier de savant.

Je pense qu'en fait vous attendez de moi que je vous parle d'autre chose, à savoir de la vocation scientifique à proprement parler. De nos jours, et au regard de l'organisation [Betrieb] scientifique, cette vocation est d'abord déterminée par le fait que la science est parvenue à un stade de spécialisation qu'elle ne connaissait pas autrefois et dans lequel elle se maintiendra à jamais, pour autant que nous puissions en juger. L'affaire ne tient pas tellement aux conditions extérieures du travail scientifique qu'aux dispositions intérieures du savant lui-même : car jamais plus un individu ne pourra acquérir la certitude d'accomplir quelque chose de vraiment parfait dans le domaine de la science sans une spécialisation rigoureuse. Tous les travaux qui empiètent sur les spécialités voisines - nous autres économistes devons passer par-là de temps à autre et cela arrive constamment et nécessairement aux sociologues - portent la marque d'une certaine résignation : nous pouvons à la rigueur poser aux spécialistes des disciplines voisines des questions utiles qu'ils n'auraient pas vues si facilement en partant de leur propre point de vue, mais en contrepartie notre travail personnel restera inévitablement incomplet. C'est uniquement grâce à cette stricte spécialisation que le travailleur scientifique pourra un jour éprouver une fois, et sans doute jamais plus une seconde fois, la satisfaction de se dire: cette fois j'ai accompli 
quelque chose qui durera. De nos jours l'œuvre vraiment définitive et importante est toujours une œuvre de spécialiste. Par conséquent, tout être qui est incapable de se mettre pour ainsi dire des œillères et de se borner à l'idée que le destin de son âme dépend de la nécessité de faire telle conjecture, et précisément celle-là, à tel endroit dans tel manuscrit, ferait mieux tout bonnement de s'abstenir du travail scientifique. Jamais il ne ressentira en lui-même [63] ce que l'on peut appeler l'« expérience » vécue de la science. Sans cette singulière ivresse dont se moquent tous ceux qui restent étrangers à la science, sans cette passion, sans cette certitude que « des milliers d'années devaient s'écouler avant que tu n'aies vu la vie et d'autres milliers d'années attendent en silence $\gg . .$. de savoir si tu es capable de faire cette conjecture-là, tu ne posséderas jamais la vocation du savant et tu ferais, .mieux de t'engager dans une autre voie. Car rien n'a de valeur, pour homme en tant qu'homme, qu'il ne peut faire avec passion.

Mais autre chose est également certain : si intense que soit cette passion, si sincère et si profonde, elle ne suffit pas, et de loin, à forcer le résultat. En vérité elle n'est qu'une condition préalable de l'« inspiration » qui seule est décisive. On rencontre aujourd'hui l'idée, largement répandue dans les milieux de la jeunesse, que la science serait devenue une opération de calcul qui se fabriquerait dans les laboratoires et les bureaux de statistique avec le seul secours du froid entendement et non avec toute « l'âme », un peu comme le travail dans une usine. À quoi il faut répondre tout de suite que ceux qui disent cela n'ont le plus souvent aucune notion claire de ce qui se passe dans une usine ou dans un laboratoire. Car dans un cas comme dans l'autre il faut que quelque chose vienne à l'esprit du travailleur - et précisément l'idée exacte - sinon il ne sera jamais capable de produire quelque chose de valable. Cette inspiration, on ne peut la forcer. Elle n'a rien à voir avec un froid calcul. Bien sûr, elle n'est elle-même de nouveau qu'une condition indispensable. Aucun sociologue par exemple ne peut se croire dispensé de faire, même pendant ses vieux jours, et des mois durant peut-être, des milliers d'opérations tout à fait triviales. Et lorsqu'on veut aboutir à un résultat on ne peut jamais impunément se décharger de ce travail sur des moyens mécaniques bien que le résultat [64] auquel on parvient soit souvent fort maigre. Toutefois, s'il ne nous vient pas à l'esprit une " idée » précise orientant nos hypothèses et si, pendant que nous faisons ces calculs, il ne nous 
vient pas une « idée » concernant la portée des résultats partiels ainsi obtenus, nous ne pourrons même pas établir ce minimum. L'inspiration ne vient normalement qu'après un travail acharné. Certes il n'en est pas toujours ainsi. Dans les sciences, l'intuition du dilettante peut avoir une portée parfaitement identique à celle du spécialiste, et même parfois plus grande. Nous devons d'ailleurs beaucoup de nos meilleures hypothèses et connaissances à des dilettantes. Ceux-ci ne se distinguent du spécialiste - suivant le jugement de Helmholtz sur Robert Mayer - que par l'absence de sûreté dans la méthode de travail et, très souvent en conséquence, par l'incapacité de vérifier, d'apprécier et d'exploiter la portée de leur intuition. Si l'inspiration ne remplace pas le travail, celui-ci de son côté ne peut remplacer ni forcer l'intuition, pas plus d'ailleurs que ne le peut la passion. Mais le travail et la passion la provoquent, et surtout les deux à la fois. Néanmoins elle ne jaillit pas quand nous le voulons, mais seulement quand elle le veut. Il est exact que nos meilleures idées nous viennent, suivant le trait de Ihering, lorsque nous sommes assis sur un canapé en train de fumer un cigare, ou bien encore, selon ce qu'Helmholtz a dit de luimême avec une précision toute scientifique, lorsque nous nous promenons sur une route qui monte légèrement, ou enfin au cours de circonstances analogues. En tout cas elles nous viennent au moment où nous ne nous y attendons pas et nullement pendant le temps où, assis à notre table de travail, nous nous creusons la cervelle et faisons des recherches. À vrai dire elles ne nous seraient pas venues si auparavant nous n'y avions pas pensé sans arrêt devant notre table de travail et si nous n'avions pas cherché avec passion une réponse.

[65]

Quoi qu'il en soit, le chercheur est obligé de Compter avec le hasard sous-jacent à tout travail scientifique : l'inspiration viendra-t-elle ou non? On peut être un travailleur remarquable et n'avoir jamais eu pourtant une inspiration originale. Par ailleurs on commettrait une grave erreur si l'on pensait qu'il n'y a que dans les sciences que les choses se passent ainsi, et que derrière un comptoir elles se présentent tout autrement que clans un laboratoire. Un commerçant ou un grand industriel sans "imagination commerciale », c'est-à-dire sans inspiration, sans intuitions géniales, ne seront leur vie durant que des hommes qui auraient mieux fait de rester commis ou techniciens : jamais ils ne créeront de nouvelles formes d'organisation. L'intuition, 
contrairement à ce que croient les pédants, ne joue pas dans les sciences un rôle plus considérable que dans les problèmes de la vie pratique dont l'entrepreneur moderne cherche à venir à bout. D'autre part - et cela aussi on l'oublie trop fréquemment - elle n'y joue pas un rôle moins important que dans l'art. C'est une idée puérile de croire qu'un mathématicien assis à sa table de travail pourrait parvenir à un résultat quelconque utile pour la science en manipulant simplement une règle ou une mécanique telle qu'une machine à calculer. L'imagination mathématique d'un Weierstrass est évidemment orientée, dans son sens et dans son résultat, tout autrement que celle d'un artiste, dont elle est également radicalement distincte du point de vue de la qualité ; mais le processus psychologique est le même dans les deux cas. Les deux ne sont qu'ivresse ( $\mu \alpha v i ́ \alpha$ au sens de Platon) et « inspiration $»$.

Les intuitions scientifiques que nous pouvons avoir dépendent donc de facteurs et de «dons » qui nous sont cachés. Cette vérité incontestable sert de prétexte, dans une certaine mentalité populaire (répandue surtout parmi les jeunes, ce qui est compréhensible), pour se mettre au service [66] d'un certain nombre d'idoles dont le culte occupe avec ostentation de nos jours une place à tous les coins de rue et dans tous les périodiques. Ces idoles sont celles de la «personnalité » et de l'" expérience vécue ». Elles ont entre elles des liens très étroits, puisqu'un peu partout règne l'idée que l'expérience vécue formerait la personnalité et qu'elle ferait partie de son essence. On se torture l'esprit pour se fabriquer des « expériences vécues », persuadé que cela constitue une attitude digne d'une personnalité, et lorsqu'on n'y réussit pas on se donne au moins l'air de posséder cette grâce. La langue allemande appelait jadis l'« expérience vécue» [Erlebnis] "sensation » [Sensation]. Mais ce qu'est la "personnalité » et ce qu'elle signifie, je crois que l'on en avait à cette époque une idée plus exacte.

Mesdames et Messieurs ! Seul l'être qui se met purement et simplement au service de sa cause possède une "personnalité » dans le monde de la science. Et ce n'est pas seulement dans ce domaine qu'il en est ainsi. Je ne connais point de grand artiste qui ait jamais fait autre chose que de se mettre au service de la cause de l'art et d'elle seule. Même une personnalité du rang de Goethe, dans la mesure où son art est en cause, a expié la liberté qu'il a prise de faire de sa 
« vie » une œuvre d'art. Ceux qui en doutent admettront ce pendant qu'il fallait justement être un Goethe pour pouvoir se permettre une chose pareille et personne ne contestera que même une personnalité de son espèce, qui n'apparaît qu'une fois tous les mille, ans, n'a pu prendre impunément une telle attitude. Il n'en va pas autrement dans le domaine de la politique, mais nous n'aborderons pas ce thème aujourd'hui. Dans le monde de la science, il est absolument impossible de considérer comme une "personnalité » un individu qui n'est que l'impresario de la cause à laquelle il devrait se dévouer, qui se produit sur la scène du monde avec l'espoir de se justifier par une "expérience vécue» [67] et qui ne se pose que ces seules questions: «Comment pourrais-je prouver que je suis autre chose qu'un simple spécialiste ? Comment pourrais-je m'y prendre pour affirmer, dans la forme et clans le fond, une chose que personne n'a encore dite ? » Il s'agit là d'un phénomène qui prend de nos jours des proportions démesurées encore qu'il ne donne que de bien piètres résultats, sans compter qu'il diminue celui qui se pose ce genre de questions. Par contre celui qui met tout son cœur à l'ouvrage, et rien qu'à cela, s'élève à la hauteur et à la dignité de la cause qu'il veut servir. Le problème se pose exactement de la même façon pour l'artiste.

En dépit de ces conditions préalables communes à la science et à l'art il y en a d'autres qui font que notre travail est profondément différent de celui de l'artiste. Le travail scientifique est solidaire d'un progrès. Dans le domaine de l'art au contraire il n'en existe pas, du moins en ce sens. Il n'est pas vrai qu'une œuvre d'art d'une époque donnée, qui met en œuvre de nouveaux moyens techniques ou encore de nouvelles lois comme celles de la perspective, serait pour ces raisons artistiquement supérieure à une autre œuvre d'art qui ignorerait ces moyens et lois, à condition évidemment que sa matière et sa forme respectent les lois mêmes de l'art, ce qui veut dire à condition que son objet ait été choisi et formé selon l'essence même de l'art bien que ne recourant pas aux moyens qui viennent d'être évoqués. Une œuvre d'art vraiment « achevée » ne sera jamais surpassée et ne vieillira jamais. Chaque spectateur pourra personnellement apprécier différemment sa signification, mais jamais personne ne pourra dire d'une œuvre vraiment « achevée » du point de vue artistique qu'elle a été " surpassée » par une autre œuvre également « achevée ». Dans le domaine de la science au contraire chacun sait que son œuvre aura 
vieilli d'ici dix, vingt ou cinquante ans. Car quel est le destin, ou plutôt la [68] signification à laquelle est soumis et subordonné, en un sens tout à fait spécifique, tout travail scientifique, comme d'ailleurs aussi tous les autres éléments de la civilisation qui obéissent à la même loi ? C'est que toute œuvre scientifique « achevée » n'a d'autre sens que celui de faire naître de nouvelles «questions »: elle demande donc à être " dépassée » et à vieillir. Celui qui veut servir la science doit se résigner à ce sort. Sans doute les travaux scientifiques peuvent garder une importance durable comme « jouissance » en vertu de leur qualité esthétique on bien comme instrument pédagogique dans l'initiation à la recherche. Mais dans les sciences, je le répète, non seulement notre destin, mais encore notre but à nous tous est de nous voir un jour dépassés. Nous ne pouvons accomplir un travail sans espérer en même temps que d'autres iront plus loin que nous. En principe ce progrès se prolonge à l'infini.

Et maintenant nous pouvons aborder le problème de la signification de la science. En effet, il n'est pas tellement évident qu'un phénomène qui obéit à cette loi du progrès possède en soi sen et raison. Pourquoi donc se livre-t-on à une occupation qui en réalité n'a jamais de fin et ne peut pas en avoir? On le fait, dit-on, en vue de buts purement pratiques on, au sens le plus large du mot, pour des buts techniques; en d'autres termes en vue d'orienter l'activité pratique en fonction des perspectives que l'expérience scientifique nous offre. Bien. Mais tout cela n'a de signification que pour l'« homme de la pratique ». La question à laquelle nous devons répondre ici est la suivante : quelle est la position personnelle de l'homme de science devant sa vocation ? - à condition évidemment qu'il la recherche pour telle. Il nous dit qu'il s'occupe de la science " pour la science même », et non pas uniquement pour que d'autres puissent en tirer des avantages commerciaux ou techniques ou encore pour que les hommes puissent [69] mieux se nourrir, se vêtir, s'éclairer et se diriger. Quelle œuvre significative espère-t-il donc accomplir grâce à ces découvertes invariablement destinées à vieillir, tout en se laissant enchaîner par cette entreprise divisée en spécialités et se perdant dans l'infini ? La réponse à cette question exige que nous apportions d'abord quelques considérations d'ordre général. 
Le progrès scientifique est un fragment, le plus important il est vrai, de ce processus d'intellectualisation auquel nous sommes soumis depuis des millénaires et à l'égard duquel certaines personnes adoptent de nos jours une position étrangement négative.

Essayons d'abord de voir clairement ce que signifie en pratique cette rationalisation intellectualiste que tous devons à la science et à la technique scientifique. Signifierait-elle par hasard que tous ceux qui sont assis dans cette salle possèdent sur leurs conditions de vie une connaissance 'supérieure à celle qu'un Indien ou un Hottentot peut avoir des siennes ? Cela est peu probable. Celui d'entre nous qui prend le tramway n'a aucune notion du mécanisme qui permet à la voiture de se mettre en marche - à moins d'être un physicien de métier. Nous n'avons d'ailleurs pas besoin de le savoir. Il nous suffit de pouvoir « compter » sur le tramway et d'orienter en conséquence notre comportement; mais nous ne savons pas comment on construit une telle machine en état de rouler. Le sauvage au contraire connaît incomparablement mieux ses outils. Lorsqu'aujourd'hui nous dépensons une somme d'argent, je parierais que chacun ou presque de mes collègues économistes, s'ils sont présents dans cette salle, donnerait une réponse différente à la question : comment se fait-il qu'avec la même somme d'argent on peut acheter une quantité de choses tantôt considérable tantôt minime ? Mais le [70] sauvage sait parfaitement comment s'y prendre pour se procurer sa nourriture quotidienne et il sait quelles sont les institutions qui l'y aident. L'intellectualisation et la rationalisation croissantes ne signifient donc nullement une connaissance générale croissante des conditions dans lesquelles nous vivons. Elles signifient bien plutôt que nous savons ou que nous croyons qu'à chaque instant nous pourrions, pourvu seulement que nous le voulions, nous prouver qu'il n'existe en principe aucune puissance mystérieuse et imprévisible qui interfère dans le cours de la vie ; bref que nous pouvons maîtriser toute chose par la prévision. Mais cela revient à désenchanter le monde. Il ne s'agit plus pour nous, comme pour le sauvage qui croit à l'existence de ces puissances, de faire appel à des moyens magiques en vue de maîtriser les esprits ou de les implorer mais de re- 
courir à la technique et à la prévision. Telle est la signification essentielle de l'intellectualisation.

D'où une nouvelle question : ce processus de désenchantement réalisé au cours des millénaires de la civilisation occidentale et, plus généralement, ce "progrès » auquel participe la science comme élément et comme moteur, ont-ils une signification qui dépasse cette pure pratique et cette pure technique ? Ce problème a été exposé avec la plus grande vigueur dans l'œuvre de Léon Tolstoï. Il y est arrivé par une voie qui lui est propre. L'ensemble de ses méditations se cristallisa de plus en plus autour du thème suivant : la mort est-elle ou non un événement qui a un sens? Sa réponse est que pour l'homme civilisé [Kulturmensch] elle n'en a pas. Et elle ne peut pas en avoir, parce que la vie individuelle du civilisé est plongée dans le «progrès » et dans l'infini et que, selon son sens immanent, une telle vie ne devrait pas avoir de fin. En effet, il y a toujours possibilité d'un nouveau progrès pour celui qui vit dans le progrès ; aucun de ceux qui [71] meurent ne parvient jamais au sommet puisque celui-ci est situé dans l'infini. Abraham ou les paysans d'autrefois sont morts « vieux et comblés par la vie » parce qu'ils étaient installés dans le cycle organique de la vie, parce que celle-ci leur avait apporté au déclin de leurs jours tout le sens qu'elle pouvait leur offrir et parce qu'il ne subsistait aucune énigme qu'ils auraient encore voulu résoudre. Ils pouvaient donc se dire "satisfaits" de la vie. L'homme civilisé au contraire, placé dans le mouvement d'une civilisation qui s'enrichit continuellement de pensées, de savoirs et de problèmes, peut se sentir « las » de la vie et non pas " comblé » par elle. En effet il ne peut jamais saisir qu'une infime partie de tout ce que la vie de l'esprit produit sans cesse de nouveau, il ne peut saisir que du provisoire et jamais du définitif. C'est pourquoi la mort est à ses yeux un événement qui n'a pas de sens. Et parce que la mort n'a pas de sens, la vie du civilisé comme telle n'en a pas non plus, puisque du fait de sa «progressivité » dénuée de signification elle fait également de la vie un événement sans signification. Dans les dernières œuvres de Tolstoï on trouve partout cette pensée qui donne le ton à son art.

Quelle position peut-on adopter à cet égard? Le "progrès » comme tel a-t-il un sens discernable dépassant la technique, de telle sorte que se mettre à son service constituerait une vocation ayant un sens ? Il est indispensable de soulever cette question. Le problème 
qui se pose alors n'est plus seulement celui de la vocation scientifique, à savoir : que signifie la science en tant que vocation pour celui qui s'y consacre ? mais un tout autre problème : quelle est la vocation de la science dans l'ensemble de la vie humaine et quelle est sa valeur?

Or sur ce point, énorme est le contraste entre le passé et le présent. Rappelez-vous la merveilleuse allégorie du début du septième livre de la [72] République de Platon, les prisonniers enchaînés de la caverne. Leur visage est tourné vers la paroi du rocher qui se dresse devant eux ; dans leur dos, la source de lumière qu'ils ne peuvent pas voir ; ils sont condamnés à ne s'occuper que des ombres que celle-ci projette sur la paroi, sans autre possibilité que celle de scruter les relations qui existent entre ces ombres. Et puis l'un d'eux réussit à briser ses chaînes ; il se retourne et voit le soleil. Ébloui, il tâtonne, il va en tous sens et il balbutie à la vue de ce qui se présente à lui. Ses compagnons le prennent pour un fou. Petit à petit il s'habitue à regarder la lumière. Cette expérience faite, son devoir est de redescendre parmi les prisonniers de la caverne afin de les conduire vers la lumière. Il est le philosophe, et le soleil représente la vérité de la science dont le but n'est pas seulement de connaître les apparences et les ombres, mais aussi l'être véritable.

Mais qui donc adopte encore de nos jours pareille attitude devant la science ? La jeunesse en particulier éprouve plutôt aujourd'hui un sentiment inverse: les constructions intellectuelles de la science constituent à ses yeux un royaume irréel d'abstractions artificielles qui s'efforcent de recueillir dans leurs mains desséchées le sang et la sève de la vie réelle, sans jamais pourtant y réussir. On croit de nos jours que c'est justement dans cette vie, qui aux yeux de Platon n'était qu'un jeu d'ombres sur la paroi de la caverne, que palpite la vraie réalité : tout le reste, estime-t-on, n'est que fantômes inanimés, détournés de la réalité, et rien d'autre. Comment s'est opérée cette transformation? L'enthousiasme passionné de Platon dans la République s'explique en dernière analyse par fait qu'à cette époque on avait découvert le sens de l'un des plus grands instruments de toute connaissance scientifique : le concept. Le mérite en revient à Socrate qui en saisit tout de suite l'importance. Mais il ne fut pas seul dans le monde à l'avoir compris. Dans les écrits [73] hindous on peut trouver des éléments d'une logique tout à fait analogue à celle d'Aristote. Mais nulle 
part ailleurs qu'en Grèce on ne trouve cette conscience de l'importance du concept. Ce furent les Grecs qui les premiers surent utiliser cet instrument, qui permettait de coincer quelqu'un dans l'étau de la logique de telle sorte qu'il ne pouvait s'en sortir qu'en reconnaissant, soit qu'il ne savait rien, soit que telle affirmation représentait la vérité et non une autre, une vérité éternelle qui ne s'effacerait jamais comme l'action et l'agitation aveugle des hommes. Ce fut là une expérience extraordinaire qui, trouve son épanouissement chez les disciples de Socrate. On crut qu'on en pouvait tirer la conséquence qu'il suffisait de découvrir le vrai concept du Beau, du Bien ou par exemple celui du Courage ou de l'Ame - ou de tout autre objet - pour être alors à même de comprendre aussitôt leur être véritable. Connaissance qui à son tour permettrait de savoir et d'enseigner comment on doit agir correctement dans la vie, et avant tout en tant que citoyen. En effet chez les Grecs, qui ne pensaient qu'à travers la catégorie de la politique, tout se ramenait à cette question. Voilà les raisons qui les amenèrent à s'occuper de la science.

À cette découverte de l'esprit hellénique s'associa par la suite à deuxième grand instrument du travail scientifique, enfanté par la Renaissance : l'expérimentation rationnelle. Elle devint le moyen éprouvé d'une expérience contrôlée sans lequel la science empirique moderne n'aurait pas été possible. Certes, on avait déjà fait des expérimentations bien avant cette date. Par exemple les expériences physiologiques aux Indes au service de la technique ascétique du Yogi ou bien les expériences mathématiques de l'antiquité hellénique à des fins de technique militaire ou enfin du Moyen Age en vue de l'exploitation des mines. Mais ce fut la Renaissance qui éleva l'expérimentation au rang d'un principe de la recherche [74] comme telle. Les précurseurs en furent sans contredit les grands novateurs dans le domaine de l'art : Léonard de Vinci et ses pareils, mais tout particulièrement et d'une façon caractéristique dans le domaine de la musique, les expérimentateurs du clavecin au $\mathrm{XVI}^{\mathrm{e}}$ siècle. De là, l'expérimentation passa dans les sciences, surtout sous l'influence de Galilée, et dans la théorie avec Bacon; elle fut ensuite adoptée par les différentes sciences exactes dans les universités du continent, d'abord et surtout en Italie et aux Pays-Bas.

Quelle a été pour ces hommes, au seuil des temps modernes, la signification de la science? Aux yeux des expérimentateurs du type de 
Léonard de Vinci et des novateurs en musique elle était le chemin qui conduit à l'art vrai, ce qui voulait dire en même temps le chemin qui conduit à la vraie nature. L'art devait être élevé au rang d'une science, ce qui signifiait en même temps et avant tout que l'artiste devait être élevé, socialement et pour lui-même, au rang d'un docteur. Cette ambition est à la base du Traité de la Peinture de Léonard de Vinci. Qu'en est-il aujourd'hui ? "La science considérée comme le chemin vers la nature ? » - une pareille phrase retentirait aux oreilles de la jeunesse comme un blasphème. Non, c'est l'inverse qui semble vrai aujourd'hui. C'est en nous libérant de l'intellectualisme de la science que nous pouvons saisir notre propre nature et par là même la nature en général! Quant à dire que la science est également le chemin qui conduit à l'art, cette opinion ne mérite pas qu'on s'y arrête. - Mais à l'époque de la formation des sciences exactes on attendait davantage encore de la science. Rappelez-vous l'aphorisme de Swammerdam : « Jje vous apporte ici, dans l'anatomie d'un pou, la preuve de la providence divine », et vous comprendrez quelle a été à cette époque la tâche propre du travail scientifique, sous l'influence (indirecte) du protestantisme et du puritanisme - trouver le chemin qui conduit à Dieu.

[75]

Toute la théologie piétiste de ce temps, surtout celle de Spener, savait que l'on n'arriverait pas à Dieu par la voie qu'avaient empruntée tous les penseurs du Moyen Age ; aussi renonça-t-elle à leur méthode philosophique, à leurs conceptions et leurs déductions. Dieu est caché, ses voies ne sont pas nos voies, ses pensées ne sont pas nos pensées. Mais on espérait découvrir les traces de ses intentions dans la nature par l'intermédiaire des sciences exactes qui nous permettraient d'appréhender physiquement ses œuvres. Et aujourd'hui ? Qui donc encore, de nos jours, croit - à l'exception de quelques grands enfants qu'on rencontre encore justement parmi les spécialistes - que les connaissances astronomiques, biologiques, physiques ou chimiques pourraient nous enseigner quelque chose sur le sens du monde ou même nous aider à trouver les traces de ce sens, si jamais il existe ? S'il existe des connaissances qui sont capables d'extirper jusqu'à la racine la croyance en l'existence de quoi que ce soit ressemblant à une « signification» du monde, ce sont précisément ces sciences-là. En définitive, comment la science pourrait-elle nous « conduire à Dieu »? 
N'est-elle pas la puissance spécifiquement a-religieuse ? Ce caractère, aucun homme - qu'il en convienne explicitement on non - ne le met plus en doute, de nos jours, en son for intérieur. La présupposition fondamentale de toute vie en communion avec Dieu pousse l'homme à s'émanciper du rationalisme et de l'intellectualisme de la science : cette aspiration ou une autre du même genre est devenue un des mots d'ordre essentiels que fait retentir cette jeunesse allemande portée vers l'émotion religieuse ou en quête d'expériences religieuses. D'ailleurs ce n'est pas après l'expérience religieuse qu'elle court, mais après l'expérience vécue en général. La seule chose déconcertante dans ce genre d'aspirations est la méthode que l'on suit, en ce sens que le seul domaine au quel l'intellectualisme n'avait encore pas touché [76] jusqu'à présent, celui de l'irrationnel, est devenu maintenant l'objet d'une prise de conscience et est examiné à la loupe. C'est à cela qu'aboutit pratiquement le romantisme intellectualiste moderne de l'irrationnel. Mais cette méthode qui se propose de nous délivrer de l'intellectualisme se traduira sans aucun doute par le résultat justement inverse de celui qu'espèrent atteindre ceux qui s'engagent dans cette voie. Enfin, bien qu'un optimisme naïf ait pu célébrer la science - c'est-à-dire la technique de la maitrise de la vie fondée sur la science - comme le chemin qui conduirait au bonheur, je crois pouvoir laisser entièrement de côté la discussion de cette question à la suite de la critique dévastatrice que Nietzsche a faite des « derniers hommes » qui « ont découvert le bonheur ». Qui donc y croit encore, à l'exception de quelques grands enfants dans les chaires des Facultés ou dans les salles de rédaction?

Revenons en arrière. Quel est alors dans ces conditions le sens de la science en tant que vocation, puisque toutes ces anciennes illusions qui voyaient en elle le chemin qui conduit à l'« Être véritable», à l'« art vrai », à la « vraie nature », au « vrai Dieu » ou au « vrai bonheur » se sont écroulées ? Tolstoï apporte la réponse la plus simple à la question en disant : elle n'a pas de sens, puisqu'elle ne donne aucune réponse à la seule question qui nous importe : "Que devonsnous faire ? Comment devons-nous vivre ?» De fait, il est incontestable qu'elle ne nous apporte pas la réponse. Aussi ne pouvons-nous porter notre interrogation que sur ce seul point : en quel sens ne nous donne-t-elle «aucune » réponse ? Et à défaut, ne pourrait-elle pas 
rendre service malgré tout à celui qui pose correctement le problème?

On a pris, de nos jours, l'habitude de parler sans cesse d'une «science sans présuppositions». Cette science existe-t-elle ? Tout dépend de ce que [77] l'on entend. Tout travail scientifique que présuppose toujours la validité des règles de la logique et de la méthodologie qui forment les fondements généraux de notre orientation dans le monde. Au regard de la question qui nous préoccupe, ces présuppositions sont ce qu'il y a de moins problématique. En outre on présuppose également que le résultat auquel aboutit le travail scientifique est important en soi, c'est-à-dire qu'il vaut la peine d'être connu [wissenswert]. Or c'est ici que se nouent manifestement tous nos problèmes, $\mathrm{cm}$ cette présupposition échappe de nouveau à toute démonstration par des moyens scientifiques. Il n'est pas possible d'interpréter le sens dernier de cette présupposition, il faut simplement l'accepter ou la refuser, suivant ses prises de position personnelles, définitives, à l'égard de la vie.

La nature de la relation entre le travail scientifique et les présuppositions qui le conditionnent varie de nouveau suivant la structure des diverses sciences. - Les sciences de la nature comme la physique, la chimie ou l'astronomie présupposent comme allant de soi qu'il vaut la peine de connaître les lois dernières du devenir cosmique, polir autant que la science est en mesure de les établir. Non seulement parce que ces connaissances nous permettent d'atteindre certains résultats techniques, mais surtout parce qu'elles ont une valeur « en soi » en tant qu'elles représentent précisément une "vocation». Néanmoins personne ne pourra jamais démontrer cette présupposition. On pourra encore bien moins prouver que le monde dont elles font la description mérite d'exister, qu'il a un « sens » ou qu'il n'est pas absurde d'y vivre. Elles ne se posent tout simplement pas ce genre de questions. - Prenons maintenant un autre exemple, celui d'une technologie aussi développée du point de vue scientifique que la médecine moderne. Exprimée de façon triviale, la «présupposition » générale de l'entre- 
prise médicale se présente ainsi : le devoir du médecin consiste dans l'obligation de [78] conserver la vie purement et simplement et de diminuer autant que possible la souffrance. Mais tout cela est problématique. Grâce aux moyens dont il dispose, le médecin maintient en vie le moribond! même si celui-ci l'implore de mettre fin à ses jours, et même si ses parents souhaitent et doivent souhaiter sa mort, consciemment on non, parce que cette vie ne représente plus aucune valeur, parce qu'ils seraient contents de le voir délivré de ses souffrances on parce que les frais pour conserver cette vie inutile - il s'agit peut-être d'un pauvre fou - deviennent écrasants. Seules les présuppositions de la médecine et du code pénal empêchent le médecin de s'écarter de cette ligne de conduite. Mais la médecine ne se pose pas la question si la vie mérite d'être vécue et dans quelles conditions? Toutes les sciences de la nature nous donnent une réponse à la question: que devons-nous faire si nous voulons être techniquement maîtres de la vie ? Quant aux questions : cela a-t-il au fond et en fin de compte un sens ? devons-nous et voulons-nous être techniquement maîtres de la vie ? elles les laissent en suspens ou bien les présupposent en fonction de leur but. - Prenons encore une autre discipline, par exemple la science de l'art. L'esthétique présuppose l'œuvre d'art. Elle se propose donc simplement de rechercher ce qui conditionne la genèse de l'œuvre d'art. Mais elle ne se demande point si le royaume de l'art n'est peut-être pas un royaume de la splendeur diabolique, un royaume de ce monde et donc dressé contre Dieu, mais également dressé contre la fraternité humaine en vertu de son esprit foncièrement aristocratique. Elle ne se pose donc pas la question : devrait-il y avoir des œuvres d'art ? - Ou encore l'exemple de la science du droit. Cette discipline établit ce qui est valable d'après les règles de la doctrine juridique, ordonnée en partie par une nécessité logique, en partie par des schèmes conventionnels donnés ; elle établit par conséquent $a ̀$ quel moment des règles de droit déterminées [79] et des méthodes déterminées d'interprétation sont reconnues comme obligatoires. Mais elle ne répond pas à la question : devrait-il y avoir un droit et devraiton instituer justement ces règles-là ? Elle peut seulement indiquer que, lorsque nous voulons un certain résultat, telle règle de droit est, d'après les normes de la doctrine juridique, le moyen approprié pour l'atteindre. - Prenons enfin l'exemple des sciences historiques. Elles nous apprennent à comprendre les phénomènes politiques, artistiques, littéraires ou sociaux de la civilisation à partir des conditions 
de leur formation. Mais elles ne donnent pas, par elles-mêmes, de réponse à la question : ces phénomènes méritaient-ils ou méritent-ils d'exister? Elles présupposent simplement qu'il y a intérêt à participer, par la pratique de ces connaissances, à la communauté des " hommes civilisés ». Mais elles ne peuvent prouver « scientifiquement» à personne qu'il y a avantage à y participer; et le fait qu'elles le présupposent ne prouve absolument pas que cela va de soi. En effet, rien de tout cela ne va de soi.

Arrêtons-nous maintenant un instant aux disciplines qui me sont familières, à savoir la sociologie l'histoire, l'économie politique, la science politique et toutes les sortes de philosophie de la culture qui ont pour objet l'interprétation des diverses sortes de connaissances précédentes. On dit, et j'y souscris, que la politique n'a pas sa place dans la salle de cours d'une université. Elle n'y a pas sa place, tout d'abord du côté des étudiants. Je déplore par exemple tout autant le fait que dans l'amphithéâtre de mon ancien collègue Dietrich Schäfer de Berlin un certain nombre d'étudiants pacifistes se soient un jour massés autour de sa chaire pour faire du vacarme, que le comportement des étudiants anti-pacifistes qui ont, semble-t-il, organisé une manifestation contre le professeur Foerster dont je suis pourtant, par mes propres conceptions, aussi éloigné que possible pour de multiples raisons. Mais la politique n'a pas [80] non plus sa place du côté des enseignants. Et tout particulièrement lorsqu'ils traitent scientifiquement les problèmes politiques. Moins que jamais alors, elle n'y a sa place. En effet, prendre une position politique pratique est une chose, analyser scientifiquement des structures politiques et des doctrines de partis en est une autre. Lorsqu'au cours d'une réunion publique, on parle de démocratie, on ne fait pas un secret de la position personnelle que l'on prend, et même la nécessité de prendre parti de façon claire s'impose alors comme un devoir maudit. Les mots qu'on utilise en cette occasion ne sont plus les moyens d'une analyse scientifique, mais ils constituent un appel politique en vue de solliciter des prises de position chez les autres. Ils ne sont plus des socs de charrue pour ameublir l'immense champ de la pensée contemplative, mais des glaives pour attaquer des adversaires, bref des moyens de combat. Ce serait une vilenie que d'employer ainsi les mots dans une salle de cours. Lorsqu'au cours d'un exposé universitaire on se propose d'étudier par exemple la « démocratie », on procède à l'examen de ses di- 
verses formes, on analyse le fonctionnement propre à chacune d'elles et on examine les conséquences qui résultent de l'une et de l'autre dans la vie; on leur oppose ensuite les formes non démocratiques de l'ordre politique et l'on essayera de pousser son analyse jusqu'au moment où l'auditeur sera lui-même en mesure de trouver le point à partir duquel il pourra prendre position en fonction de ses propres idéaux fondamentaux. Mais le véritable professeur se gardera bien d'imposer à son auditoire, du haut de la chaire, une quelconque prise de position, que ce soit ouvertement ou par suggestion - car la manière la plus déloyale est évidemment celle qui consiste à a laisser parler les faits $\gg$.

Pour quelles raisons, au fond, devons-nous nous en abstenir? je présume qu'un certain nombre de mes honorables collègues seront d'avis qu'il est [81] en général impossible de mettre en pratique cette réserve personnelle, et que même si la chose 'Était possible, ce serait une marotte que de prendre pareilles précautions. Dame! on ne peut démontrer scientifiquement à personne en quoi consiste son devoir de professeur d'université. On ne peut jamais exiger de lui que la probité intellectuelle, ce qui veut dire l'obligation de reconnaître que d'une part l'établissement des faits, la détermination des réalités mathématiques et logiques ou la constatation des structures intrinsèques des valeurs culturelles, et d'autre part la réponse aux questions concernant la valeur de la culture et de ses contenus particuliers ou encore celles concernant la manière dont il faudrait agir dans la cité et au sein des groupements politiques, constituent deux sortes de problèmes totalement hétérogènes. Si l'on me demandait maintenant pourquoi cette dernière série de questions doit être exclue d'un amphithéâtre, je répondrai que le prophète et le démagogue n'ont pas leur place dans une chaire universitaire. Il est dit au prophète aussi bien qu'au démagogue : "Va dans la rue et parle en publie », ce qui veut dire là où l'on peut te critiquer. Dans un amphithéâtre au contraire on fait face à son auditoire d'une tout autre manière : le professeur y a la parole, mais les étudiants sont condamnés au silence. Les circonstances veulent que les étudiants soient obligés de suivre les cours d'un professeur en vue de Leur future carrière et qu'aucune personne présente dans la salle de cours ne puisse critiquer le maître. Aussi un professeur est-il inexcusable de profiter de cette situation pour essayer de marquer ses élèves de ses propres conceptions politiques au lieu de 
leur être utile, comme il en a le devoir, par l'apport de ses connaissances et de son expérience scientifique. Il peut certes arriver que tel ou tel professeur ne réussisse qu'imparfaitement à faire taire ses préférences. Dans ce cas il s'expose à la critique la plus sévère dans le for de sa propre conscience. Mais une telle défaillance ne prouve absolument rien, car [82] il existe bien d'autres défaillances, par exemple les erreurs matérielles qui ne prouvent non plus rien contre l'obligation de rechercher la vérité. Au surplus c'est justement au nom de l'intérêt de la science que je condamne cette façon de procéder. Je suis prêt à vous fournir la preuve au moyen des œuvres de nos historiens que, chaque fois qu'un homme de science fait intervenir son propre jugement de valeur, il n'y a plus compréhension intégrale des faits. Mais cette démonstration déborderait le cadre du thème qui nous occupe ce soir et exigerait de trop longues discussions.

Je voudrais seulement vous poser cette simple question : Comment, dans une leçon qui a pour objet l'étude des diverses formes des États et des Églises on l'histoire des religions, est-il possible d'amener d'un côté un catholique croyant et de l'autre un franc-maçon à soumettre ces phénomènes aux mêmes critères d'évaluation ? Cela est tout bonnement exclu. Et pourtant le professeur doit avoir l'ambition et même se faire un devoir d'être utile à l'un et à l'autre par ses connaissances et sa méthode. Vous pouvez m'objecter à juste titre que le catholique croyant n'acceptera jamais la manière de comprendre l'histoire des origines du christianisme telle que l'expose un professeur qui ne partage pas ses présuppositions dogmatiques. Cela est certain ! La raison de leurs divergences vient de ce que la science «sans présuppositions », en tant qu'elle refuse la soumission à une autorité religieuse, ne connaît en fait ni « miracle » ni « révélation ». Sinon elle serait infidèle à ses propres présuppositions. Mais le croyant connaît les deux positions. Cette science "sans présuppositions » exige de sa part rien de moins - mais également rien de plus que le souci de reconnaître simplement que, si le cours des choses doit être expliqué sans l'intervention d'aucun de ces éléments surnaturels auxquels l'explication empirique refuse tout caractère causal, il ne peut être expliqué [83] autrement que par la méthode que la science s'efforce d'appliquer. Et le croyant peut admettre cela sans aucune infidélité à sa foi. 
Mais alors une nouvelle question se pose le travail accompli par la science n'aurait-il finalement aucun sens aux yeux de celui qui reste indifférent aux faits comme tels et qui n'accorde d'importance qu'aux prises de position pratique ? je crois que même dans ce cas elle n'est pas dénuée de signification. Premier point à signaler : la tâche primordiale d'un professeur capable est d'apprendre à ses élèves à reconnaître qu'il y a des faits inconfortables, j'entends par là des faits qui sont désagréables à l'opinion personnelle d'un individu; en effet il existe des faits extrêmement désagréables pour chaque opinion, y compris la mienne. Je crois qu'un professeur qui oblige ses élèves à s'habituer à ce genre de choses accomplit plus qu'une œuvre purement intellectuelle, je n'hésite pas à prononcer le mot d'« œuvre morale », bien que cette expression puisse peut-être paraître trop pathétique pour, désigner une évidence aussi banale.

Je n'ai parlé jusqu'à présent que des raisons pratiques qui justifient le refus d'imposer ses convictions personnelles. Il y en a encore d'autres. L'impossibilité de se faire le champion de convictions pratiques « au nom de la science » - hormis le seul cas qui porte sur la discussion des moyens nécessaires pour atteindre une fin fixée au préalable - tient à des raisons beaucoup plus profondes. Une telle attitude est en principe absurde parce que divers ordres de valeurs s'affrontent dans le monde en une lutte inexpiable. Sans vouloir faire autrement l'éloge de la philosophie du vieux Mill ', il faut néanmoins reconnaître qu'il a raison de dire que lorsqu'on part de l'expérience pure, on aboutit au polythéisme. La formule a un aspect superficiel et même paradoxal, et pourtant elle contient une part de vérité. S'il est une chose que de nos jours nous [84] n'ignorons plus, c'est qu'une chose peut être sainte non seulement bien qu'elle ne soit pas belle mais encore parce que et dans la mesure où elle n'est pas belle - vous en trouverez les références au chapitre LIII du livre d'Isaïe et dans le psaume 21. De même une chose peut être belle non seulement bien qu'elle ne soit pas bonne, niais précisément par ce en quoi elle n'est pas bonne. Nietzsche nous l'a réappris, mais avant lui Baudelaire l'avait déjà dit dans les Fleurs $d u$ Mal, c'est là le titre qu'il a choisi pour son œuvre poétique. Enfin la sagesse populaire nous enseigne qu'une chose peut être vraie bien qu'elle ne soit et alors qu'elle n'est ni belle ni sainte ni bonne. Mais ce ne sont là que les cas les plus élé-

1 James Mill. [Note ajoutée par JMT.] 
mentaires de la lutte qui oppose les dieux des différents ordres et des différentes valeurs. J'ignore comment on pourrait s'y prendre pour trancher "scientifiquement» la question de la valeur de la culture française comparée à la culture allemande; car là aussi différents dieux se combattent, et sans doute pour toujours. Les choses ne se passent donc pas autrement que dans le monde antique, encore sous le charme des dieux et des démons, mais prennent un sens différent. Les Grecs offraient des sacrifices d'abord à Aphrodite, puis à Apollon et surtout à chacun des dieux de la cité ; nous faisons encore de même de nos jours, bien que notre comportement ait rompu le charme et se soit dépouillé du mythe qui vit cependant en nous [der mythischen aber innerlich wahren Plastik]. C'est le destin qui gouverne les dieux et non pas une science, quelle qu'elle soit. Tout ce qu'il nous est donné de comprendre, c'est ce que le divin signifie pour une société donnée, ou ce que l'une ou l'autre société considère comme tel. Voilà la limite de la discussion qu'un professeur ne peut dépasser au cours d'une leçon, ce qui évidemment ne veut pas dire qu'on aurait ainsi résolu l'immense problème de la vie qui se cache derrière ces questions. Mais [85] alors d'autres puissances que celles d'une chaire universitaire entrent en jeu. Quel est l'homme qui aurait la prétention de réfuter a scientifiquement » l'éthique du Sermon sur la Montagne, ou par exemple la maxime «n'oppose pas de résistance au mal », ou encore la parabole des deux joues? Il est pourtant clair que, du point de vue strictement humain, ces préceptes évangéliques font l'apologie d'une éthique qui va contre la dignité. À chacun de choisir entre la dignité de la religion, que nous procure cette éthique, et la dignité d'un être viril qui prêche tout autre chose, à savoir a résiste au mal, sinon tu es responsable de sa victoire ». Suivant les convictions profondes de chaque être, l'une de ces éthiques prendra le visage du diable, l'autre celle du dieu et chaque individu aura à décider, de son propre point de vue, qui est dieu et qui est diable. Il en est ainsi dans tous les ordres de la vie. Le rationalisme grandiose, sousjacent à la conduite sciemment éthique de notre vie qui jaillit de toutes les prophéties religieuses, a détrôné le polythéisme au profit de l'« Unique dont nous avons besoin »; mais dès qu'il fut lui-même aux prises avec la réalité de la vie intérieure et extérieure il s'est vu contraint de consentir aux compromis et aux accommodements dont nous a tous instruits l'histoire du christianisme. Mais la religion est devenue de nos jours « routine quotidienne ». La multitude des dieux 
antiques sortent de leurs tombes, sous la forme de puissances impersonnelles parce que désenchantées, et ils s'efforcent à nouveau de faire retomber notre vie en leur pouvoir tout en reprenant leurs luttes éternelles. D'où les tourments de l'homme moderne qui se révèlent tout particulièrement pénibles pour la jeune génération : comment se montrer à la hauteur du quotidien ? Toutes les quêtes d'« expériences vécues » ont leur source dans cette faiblesse, car c'est faiblesse que n'être pas capable de regarder en face le sévère destin de son temps.

[86]

Tel est le destin de notre civilisation : il nous faut à nouveau prendre plus clairement conscience de ces déchirements que l'orientation prétendue exclusive de notre vie en fonction du pathos grandiose de l'éthique chrétienne avait réussi à masquer pendant mille ans.

Mais nous en avons assez dit sur ce sujet qui risque de nous entraîner trop loin. L'erreur que commet une partie de notre jeunesse quand elle répond à tout ce que nous venons de dire par cette réplique : "Soit ! Mais si nous assistons à vos cours, c'est pour entendre autre chose que des analyses et des déterminations de faits ", l'erreur qu'elle commet en ce cas consiste à chercher dans le professeur autre chose qu'un maître face à ses élèves : elle espère trouver un chef et non un professeur. Or c'est uniquement en tant que professeur que nous occupons une chaire. Il ne faut donc pas confondre deux choses si totalement différentes et l'on peut facilement se convaincre de la nécessité de cette distinction. Permettez-moi de vous conduire encore une fois en Amérique, parce que l'on peut y observer un certain nombre de choses dans leur forme originelle la plus frappante. Le jeune Américain apprend beaucoup moins de choses que le jeune Allemand. Cependant, malgré un nombre incroyable d'examens, il n'est pas encore devenu, à cause de l'esprit qui règne dans l'université américaine, la pure bête à examens qu'est l'étudiant allemand. En effet, la bureaucratie qui fait du diplôme une condition préalable, une sorte de billet d'entrée dans le royaume de la prébende des emplois, n'est encore qu'à ses débuts outre-Atlantique. Le jeune Américain ne respecte rien ni personne, ni tradition ni situation professionnelle, mais il s'incline devant la prouesse personnelle d'un quelconque individu. Cela, il l'appelle " démocratie ». Aussi caricaturale que puisse paraître la réalité américaine lorsqu'on la compare à la signification vraie du mot démocratie, [87] c'est ce sens qu'il lui donne et cela seul 
est important pour le moment. Il se fait de son professeur une idée simple : celui-ci lui vend des connaissances et des méthodes pour l'argent de son père, exactement comme la marchande de légumes vend des choux à sa mère. Rien d'autre. Si le professeur est par exemple un champion de football, on n'hésitera pas, il est vrai, à le considérer comme un chef dans ce domaine précis. Mais s'il ne l'est pas (ou s'il n'est pas quelque chose de similaire dans un autre sport), il n'est qu'un professeur et rien de plus. Il ne viendrait jamais à l'idée du jeune Américain que son professeur pourrait lui vendre des « conceptions du monde » ou des règles valables pour la conduite de la vie. Bien sur, nous rejetons une pareille conception, ainsi formulée. Cependant on peut se demander si cette façon de voir, qu'à dessein j'ai grossie quelque peu, ne contient pas un grain de vérité.

Mes chers étudiants! Vous venez à nos cours en exigeant de nous, qui sommes vos professeurs, des qualités de chef sans jamais songer au préalable que sur cent professeurs, quatre-vingt-dix-neuf n'ont pas et ne doivent pas avoir la prétention d'être des champions de football de la vie ni non plus des « chefs » dans les affaires qui concernent la conduite de notre vie. Il ne faut tout de même pas oublier que la valeur d'un être humain ne dépend pas fatalement des qualités de chef qu'il peut ou ne peut pas posséder En tout cas, les dispositions qui font d'un homme un savant éminent et un professeur d'université ne sont certainement pas les mêmes que celles qui pourraient faire de lui un chef dans le domaine de la conduite pratique de la vie, et spécialement dans le domaine pratique. Qu'un homme possède cette dernière qualité, cela relève du pur hasard. Si chaque professeur qui occupe une chaire avait le sentiment d'être placé devant l'impudente exigence de montrer qu'il est un [88] chef, cela deviendrait très inquiétant. Et la chose deviendrait encore plus inquiétante si on laissait chaque professeur d'université juge de la possibilité de jouer ce rôle dans l'amphithéâtre. En effet, les individus qui se prennent volontiers pour des chefs sont le plus souvent les moins aptes à cette fonction : la salle où le professeur est devant son pupitre n'est en tout cas jamais l'endroit où il pourrait faire preuve d'une telle aptitude. Le professeur qui se sent la vocation de conseiller la jeunesse et qui jouit de sa confiance doit s'acquitter de ce rôle dans le contact personnel d'homme à homme. S'il se sent appelé participer aux luttes entre les conceptions du monde et les opinions des partis, il lui est loisible de le faire hors 
de la salle de cours, sur la place publique, c'est-à-dire dans la presse, dans les réunions publiques, dans les associations, bref partout où il le voudra. Il est en effet par trop commode de montrer son courage de partisan en un endroit où les assistants, et peut-être les opposants, sont condamnés au silence.

Finalement vous me direz s'il en est ainsi, quel est alors, au fond, l'apport positif de la science à la « vie » pratique et personnelle? Cette question met à nouveau sur le tapis le problème de la « vocation » de la science en elle-même. Premièrement la science met naturellement à notre disposition un certain nombre de connaissances qui nous permettent de dominer techniquement la vie par la prévision, aussi bien dans le domaine des choses extérieures que dans celui de l'activité des hommes. Vous me répliquerez : après tout, cela n'est rien d'autre que la marchande de légumes du jeune Américain. Tout à fait d'accord. En second lieu, elle nous apporte quelque chose que la marchande de légumes ne peut à coup sûr nous donner : des méthodes de [89] pensée, c'est-à-dire des instruments et une discipline. Vous me rétorquerez peut-être qu'il ne s'agit plus cette fois-ci de légumes, mais de quel, que chose qui n'est qu'un moyen pour se procurer des légumes. Soit! Admettons-le en attendant. Mais nous ne sommes heureusement pas encore arrivés au bout du compte. Nous sommes encore en mesure de vous aider à y trouver un troisième avantage : la science contribue à une œuvre de clarté. À condition évidemment que nous, savants, nous la possédions d'abord. nous-mêmes. S'il en est ainsi, nous pouvons vous indiquer clairement qu'en présence de tel problème de valeur qui est en jeu on peut adopter pratiquement telle position ou telle autre - je vous prie, pour simplifier, de prendre des exemples dans les situations sociales auxquelles nous avons à faire face. Quand on adopte alors telle ou telle position il faudra, suivant la procédure scientifique, appliquer tels ou tels moyens pour pouvoir mener à bonne fin son projet. Il peut arriver qu'à ce momentlà les moyens présentent par eux-mêmes un caractère qui nous oblige à les refuser. Dans ce cas il nous faudra justement choisir entre la fin et les moyens inévitables que celle-ci exige. La fin «justifie-t-elle » 
les moyens on non? Le professeur peut seulement vous montrer la nécessité de ce choix, mais il ne peut faire davantage s'il se limite à son rôle de professeur et s'il ne veut pas devenir un démagogue. En outre, il peut également vous indiquer que, lorsque vous voulez, telle ou telle fin, il faudra consentir à telles on telles conséquences subsidiaires qui en résulteront suivant les leçons de l'expérience. Dans ce cas peuvent alors se présenter les mêmes difficultés qu'à propos du choix des moyens. À ce niveau nous n'avons pourtant affaire qu'à des problèmes qui peuvent également se présenter à n'importe quel technicien ; celui-ci est contraint, dans de nombreux cas, de se décider selon le principe du moindre mal ou celui du relativement [90] meilleur. Avec cette différence cependant : une chose est d'ordinaire donnée préalablement au technicien, et même la chose capitale, le but. Or lorsqu'il s'agit de problèmes fondamentaux, le but ne nous est pas donné. Grâce à cette remarque nous pouvons définir maintenant l'ultime apport de la science au service de la clarté, apport au-delà duquel il n'y en a plus d'autres. Les savants peuvent - et doivent - encore vous dire que tel ou tel parti que vous adoptez dérive logiquement, et en toute conviction, quant à sa signification, de telle ou telle vision dernière et fondamentale du monde. Une prise de position peut ne dériver que d'une seule vision du monde mais il est également possible qu'elle dérive de plusieurs, différentes entre elles. Ainsi le savant peut vous dire que votre position dérive de telle conception et non d'une autre. Reprenons la métaphore que nous avons utilisée tout à l'heure. La science vous indiquera qu'en adoptant telle position vous servirez tel Dieu et vous offenserez tel autre parce que, si vous restez fidèles à vous-mêmes, vous en viendrez nécessairement à telles conséquences internes, dernières et significatives. Voilà ce que la science peut apporter, du moins en principe. C'est également cette œuvre que cherchent à accomplir la discipline spéciale qu'on appelle philosophie et les méthodologies particulières aux autres disciplines. Si nous sommes, en tant que savants, à la hauteur de notre tâche (ce qu'il faut évidemment présupposer ici), nous pouvons alors obliger l'individu $a ̀$ se rendre compte du sens ultime de ses propres actes, ou du moins l'y aider. Il nie semble que ce résultat n'est pas tellement négligeable, même en ce qui concerne la vie personnelle. Lorsqu'un professeur obtient ce résultat je suis alors enclin à dire qu'il est au service de puissances «morales », à savoir le devoir de faire naître en l'âme des autres la clarté et le sens de la responsabilité. Je crois qu'il lui sera 
d'autant plus facile d'accomplir [91] cette œuvre qu'il évitera par scrupule d'imposer ou de suggérer personnellement à ses auditeurs une conviction.

Les opinions que j'expose présentement devant vous ont, en vérité, pour base la condition fondamentale suivante : pour autant que la vie a en elle-même un sens et qu'elle se comprend d'elle-même, elle ne connait que le combat éternel que les dieux se font entre eux ou, en 'évitant la métaphore, elle ne connaît que l'incompatibilité des points de vue ultimes possibles, l'impossibilité de régler leurs conflits et par conséquent la nécessité de se décider en faveur de l'un ou de l'autre. Quant à savoir si dans ces conditions il vaut la peine pour quelqu'un de faire de la science sa " vocation » ou bien si elle constitue en elle-même une " vocation » objectivement valable, il faut reconnaître que ce genre de questions implique à son tour un jugement de valeur sur lequel on ne peut se prononcer dans un amphithéâtre. En effet la réponse affirmative à ces questions constitue précisément la présupposition de l'enseignement. Personnellement j'y réponds affirmativement par mes propres travaux. Mais tout cela vaut également, et même tout particulièrement, pour le point de vue foncièrement hostile à l'intellectualisme qui, à la manière de la jeunesse moderne, voit ou la plupart du temps s'imagine voir en lui le diable le plus dangereux. C'est peut-être le moment de rappeler à cette jeunesse la sentence: "N'oublie pas que le diable est vieux, deviens donc vieux toi aussi pour pouvoir le comprendre ». Ce qui ne veut pas dire qu'il soit nécessaire de prouver son âge en produisant un acte de naissance. Le sens de ces paroles est tout autre : si tu veux avoir raison de ce genre de diable, il ne faut pas prendre la fuite, comme cela arrive trop fréquemment de nos jours, mais il te faut d'abord examiner à fond ses voies pour connaître sa puissance et ses limites.

La science est de nos jours une "vocation » fondée [92] sur la spécialisation au service de la prise de conscience de nous-mêmes et de la connaissance des rapports objectifs. Elle n'est pas une grâce qu'un visionnaire on un prophète auraient reçue en vue de dispenser le salut de l'âme, ou des révélations ; elle n'est pas non plus partie intégrante de la méditation des sages et clés philosophes qui s'interrogent sur le sens du monde. Telle est la donnée inéluctable de notre situation historique à laquelle nous ne pouvons pas échapper si nous restons fidèles à nous-mêmes. Et maintenant si vous posez à nouveau 
la question à la manière de Tolstoï en disant : «Étant donné la défaillance de la science, qui pourra nous donner une réponse à la question : que devons-nous faire et comment devons-nous organiser notre vie? ", ou bien si vous posez le problème dans les termes que nous avons employés ce soir : "Quel Dieu devons-nous servir parmi tous ceux qui se combattent? devons-nous peut-être servir un tout autre Dieu, mais quel est-il ? », -je vous dirai : adressez-vous à un prophète ou à un sauveur. Et si ce sauveur n'existe plus ou bien si son message n'est plus entendu, soyez certains que vous ne réussirez pas à le faire descendre sur terre tout simplement parce que des milliers de professeurs, transformés en petits prophètes privilégiés et stipendiés par l'État, essayent de jouer ce rôle dans un amphithéâtre. Par ce moyen vous ne réussirez qu'à faire une chose, à empêcher la jeune génération de se rendre compte de ce fait décisif : lé prophète, que tant de membres de la jeune génération appellent de tous leurs vœux, n'existe pas. Au surplus vous les empêcherez de saisir toute la signification de cette absence. Je suis certain que l'on ne rend aucun service à un individu qui « vibre » à la religion quand on lui cache, ainsi qu'aux autres hommes d'ailleurs, que son destin est de vivre en une époque indifférente à Dieu et aux prophètes, ou encore quand on lui dissimule cette situation fondamentale au moyen de ces succédanés que sont toutes les prophéties [93] faites du haut d'une chaire universitaire. Il me semble que le croyant, dans la pureté de sa foi, devrait s'insurger contre une pareille duperie.

Mais il vous vient peut-être maintenant à l'idée une autre question : quelle position faut-il adopter devant le fait qu'il y a une théologie qui prétend au titre de «science»? Nous n'allons pas tourner autour du pot et esquiver la question. Il est certain que l'on ne trouve pas partout dans le monde une " théologie » et des " dogmes ", mais il est tout aussi certain qu'on en trouve également ailleurs que dans le christianisme. Ainsi, en remontant dans l'histoire, on trouvé des théologies largement développées dans l'Islam, le manichéisme, la gnose, l'orphisme, le parsisme et le bouddhisme, dans les sectes hindouistes, dans le taoïsme et dans les Upanishads et naturellement aussi dans le judaïsme. Elles ont pris chaque fois un développement systématique extrêmement différent. Ce n'est pourtant pas un effet du hasard si le christianisme occidental a non seulement élaboré on cherché à élaborer de façon plus systématique sa théologie - contrairement aux élé- 
ments de théologie que l'on trouve dans le judaïsme - mais lui a encore donné un développement dont la signification historique est de loin la plus considérable. Cela s'explique par l'influence de l'esprit hellénique, car toute théologie occidentale procède de cet esprit, comme toute théologie orientale procède manifestement de la pensée hindoue. La théologie est une, rationalisation intellectuelle de l'inspiration religieuse. Nous avons déjà dit qu'il n'existe pas de science entièrement exempte de présuppositions et qu'aucune science ne peut apporter la preuve de sa valeur à qui rejette ses présuppositions. Mais la théologie ajoute encore d'autres présuppositions qui lui sont propres, principalement en ce qui concerne son travail et la justification de son existence. Naturellement, dans un sens et dans une mesure très variables. Assurément toute théologie, même la théologie [94] hindoue, accepte la présupposition que le monde doit avoir un sens, mais la question qui se pose est de savoir comment il faut interpréter ce sens pour pouvoir le penser. Il s'agit là d'une démarche identique à celle de la théorie de la connaissance de Kant, qui, partant de la présupposition a la vérité scientifique existe et elle est valide », se demande ensuite quelles sont les présuppositions qui la rendent possible. Ou encore elle rappelle la démarche des esthéticiens modernes qui partent (explicitement, comme par exemple G.V. Lukâcs, ou effectivement) de la présupposition « il existe des œuvres d'art » et qui se demandent ensuite comment cela est possible. Il est vrai qu'en règle générale les théologies ne se contentent pas de cette dernière présupposition qui relève essentiellement de la philosophie de la religion. Elles partent généralement d'autres présuppositions supplémentaires : d'une part, qu'il faut croire à certaines " révélations » qui sont importantes pour le salut de l'âme - c'est-à-dire des faits qui seuls rendent possible une conduite de la vie ayant un sens - et d'autre part qu'il existe certains états et activités qui possèdent le caractère de la sainteté - c'est-à-dire qui constituent une conduite qui soit compréhensible du point de vue de la religion, ou du moins de ses éléments essentiels. Mais à son tour la théologie se heurte à la question : comment peut-on comprendre, en fonction de notre représentation totale du monde, ces présuppositions que nous ne pouvons guère qu'accepter? Elle nous répond qu'elles appartiennent à une sphère qui se situe au-delà des limites de la "science ». Elles ne constituent donc pas un « savoir » au sens habituel du mot, mais un "avoir » [Haben], en ce sens qu'aucune théologie ne peut suppléer à la foi et aux autres élé- 
ments de sainteté chez celui qui ne les «possède » pas. À plus forte raison aucune autre science ne pourra le faire. Au contraire dans toute théologie «positive » le croyant aboutit nécessairement à un moment [95] donné à un point où il ne pourra faire autrement qu'appliquer la maxime de saint Augustin : Credo non quod, sed quia absurdum est. Le pouvoir d'accomplir cette prouesse de virtuose, qu'est le « sacrifice de l'intellect» constitue le trait caractéristique et décisif de tout homme pratiquant. S'il en est ainsi, on voit que, malgré la théologie (ou plutôt à cause d'elle), il existe une tension insurmontable (que précisément la théologie nous révèle) entre le domaine de la croyance à la « science » et celui du salut religieux.

Seul le disciple fait légitimement le « sacrifice de l'intellect» en faveur du prophète, comme également le croyant en faveur de l'Église. Mais jamais encore on n'a vu naître une nouvelle prophétie (je répète à dessein cette métaphore qui a pu choquer quelques-uns d'entre vous) parce que certains intellectuels modernes éprouvent le besoin de meubler leur âme d'objets anciens pour ainsi dire garantis authentiques, auxquels ils ajoutent également la religion que d'ailleurs ils ne pratiquent pas, tout simplement parce qu'ils se remémorent qu'elle fait partie de ce bric-à-brac. Ils substituent ainsi à la religion un succédané dont ils parent leur âme comme une chapelle privée, ornée à plaisir de toutes les bondieuseries recueillies dans toutes les parties du monde. Ou encore ils se créent un substitut de toutes les formes possibles d'expériences vécues auxquelles ils confèrent la dignité de la sainteté mystique pour les colporter ensuite sur le marché des livres. Or tout cela n'est que du charlatanisme et une façon de s'illusionner soi-même. Mais il y a un autre phénomène qui n'a rien du charlatanisme et qui consiste au contraire en quelque chose de très sérieux et de très sincère, bien que parfois on, interprète peut-être faussement sa signification. Je veux parler de ces mouvements de jeunesse qui se sont développés au cours des dernières années dans le but de donner aux relations humaines personnelles à l'intérieur d'une communauté le sens d'une relation religieuse, cosmique [96] ou mystique. S'il est vrai que tout acte de véritable fraternité peut aller de pair avec la conscience d'ajouter ainsi quelque chose d'impérissable au monde des relations supra-personnelles, il me semble par contre douteux que la dignité des relations communau- 
taires puisse être rehaussée par ces interprétations religieuses. Ces considérations cependant nous éloignent de notre sujet.

Le destin de notre époque caractérisée par la rationalisation, par l'intellectualisation et surtout par le désenchantement du monde, a conduit les humains à bannir les valeurs suprêmes les plus sublimes de la vie publique. Elles ont trouvé refuge soit dans le royaume transcendant de la vie mystique soit dans la fraternité des relations directes et réciproques entre individus isolés. Il n'y a rien de fortuit dans le fait que l'art le plus éminent de notre temps est intime et non monumental, ni non plus dans le fait que de nos jours on retrouve uniquement dans les petits cercles communautaires, dans le contact d'hommes à hommes, en pianissimo, quelque chose qui pourrait correspondre au pneuma prophétique qui embrasait autrefois les grandes communautés et les soudait ensemble. Lorsque nous essayons d'« inventer » à tout prix un nouveau style de l'art monumental, nous aboutissons à ces lamentables horreurs que sont les monuments des vingt dernières années. Et lorsque nous nous creusons la cervelle pour fabriquer de nouvelles religions, nous aboutissons intérieurement, en l'absence de toute prophétie nouvelle et authentique, à quelque chose d'analogue qui aura pour notre âme des effets encore plus désastreux. Les prophéties qui tombent des chaires universitaires n'ont finalement d'autres résultats que de former des sectes de fanatiques, mais jamais de véritables communautés. À celui qui ne peut supporter avec virilité ce destin de notre époque on ne peut que donner le conseil suivant : retourne en silence, sans donner à ton geste la publicité habituelle des renégats, mais simplement et sans façons, [97] dans les bras largement ouverts et pleins de miséricorde des vieilles Églises. Celles-ci ne lui rendront pas le retour pénible. Car de toute manière, quoi qu'il fasse, il serait contraint de faire le " sacrifice de son intellect », cela est inévitable. Ce n'est pas moi qui le lui reprocherai s'il a vraiment la force de le faire. En effet, sacrifier pour se donner sans conditions à une religion vaut moralement mieux que l'art d'éluder la simple probité intellectuelle, qui est le lot de celui qui n'a pas le courage de chercher à voir clair pour lui-même dans les choix derniers et qui se facilite la tâche en consentant à un relativisme précaire. À mes yeux, ce don de soi est plus louable que toutes ces prophéties d'universitaires qui ne voient pas clairement que dans un amphithéâtre aucune vertu n'a plus de valeur que celle de la probité intellectuelle. Cette 
probité nous commande de constater que tous ceux-là, et ils sont nombreux, qui de nos jours vivent dans l'attente de nouveaux prophètes et de 'nouveaux sauveurs se trouvent dans la situation décrite par ce beau chant édomite du veilleur du temps de l'exil, chant qui a été recueilli dans les oracles d'Isaïe :

On me crie de Séïr

«Veilleur, où en est la nuit?

Veilleur, où en est la nuit?»

Le veilleur répond :

«Vient le matin, et puis la nuit.

Si vous le voulez, interrogez,

Convertissez-vous, revenez! »

Le peuple auquel on a dit cela n'a cessé de poser la question et de vivre dans l'attente depuis plus de deux mille ans, et nous connaissons son bouleversant destin. Tirons-en la leçon ! Rien ne s'est [98] encore fait par la seule ferveur et par l'attente. Il faut s'y prendre autrement et se mettre à son travail et répondre aux demandes de chaque jour - dans sa vie d'homme, mais aussi dans son métier. Et ce travail sera simple et facile si chacun trouve le démon qui tient les fils de sa vie et lui obéit. 
[99]

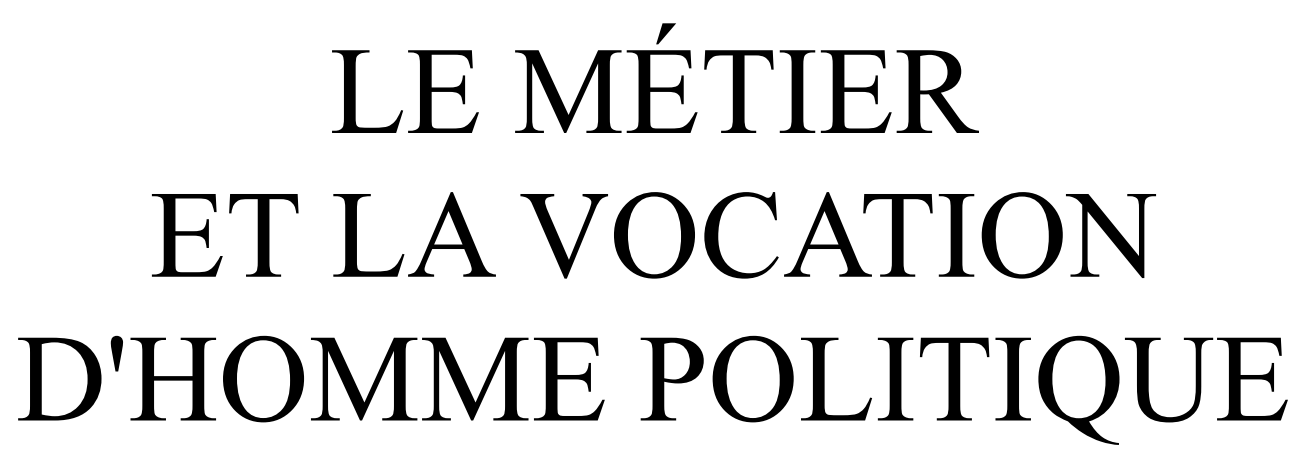

1919

Politik als Beruf

$\underline{\text { Retour à la table des matières }}$

Cette conférence que vous m'avez prié de faire vous décevra nécessairement pour plusieurs raisons. Dans une causerie qui a pour thème la vocation politique vous vous attendez instinctivement à ce que je prenne position sur les problèmes du jour. Or je ne m'y attaquerai qu'à la fin de mon exposé et d'une manière purement formelle, au moment d'aborder certains problèmes concernant la signification de l'activité politique dans l'ensemble de la conduite humaine. Nous excluons donc entièrement de notre propos toutes les questions de cet ordre : quelle politique devons-nous faire ? ou encore : quels contenus devons-nous donner à notre propre activité politique ? En effet, ces questions n'ont aucun rapport avec le problème général que nous nous posons ici, à savoir : qu'est-ce que la vocation politique et quel sens peut-elle revêtir? Maintenant, attaquons notre sujet. 
Qu'entendons-nous par politique ? Le concept est extraordinairement vaste et embrasse toutes les espèces d'activité directive [leitende] autonome. On parle de la politique de devises d'une banque, de la politique d'escompte de la Reichsbank, de la politique d'un syndicat au cours d'une grève ; on peut également parler de la politique scolaire d'une commune urbaine ou rurale, de la politique d'un comité qui dirige une association, et finalement de [100] la politique d'une femme habile qui cherche à gouverner son mari. Nous ne donnerons évidemment pas une signification aussi vaste au concept qui servira de base aux réflexions que nous ferons ce soir. Nous entendrons uniquement par politique la direction du groupement politique que nous appelons aujourd'hui « État », ou l'influence que l'on exerce sur cette direction.

Mais qu'est-ce donc qu'un groupement "politique » du point de vue du sociologue? Qu'est-ce qu'un État? Lui non plus ne se laisse pas définir sociologiquement par le contenu de ce qu'il fait. Il n'existe en effet presque aucune tâche dont ne se soit pas occupé un jour un 'groupement politique quelconque ; d'un autre côté il n'existe pas non plus de tâches dont on puisse dire qu'elles aient de tout temps, du moins exclusivement, appartenu en propre aux groupements politiques que nous appelons aujourd'hui États ou qui ont été historiquement les précurseurs de l'État moderne. Celui-ci ne se laisse définir sociologiquement que par le moyen spécifique qui lui est propre, ainsi qu'à tout autre groupement politique, à savoir la violence physique.

« Tout État est fondé sur la force », disait un jour Trotsky à BrestLitovsk. En effet, cela est vrai. S'il n'existait que des structures sociales d'où toute violence serait absente, le concept d'État aurait alors disparu et il ne subsisterait que ce qu'on appelle, au sens propre du terme, l'« anarchie ». La violence n'est évidemment pas l'unique moyen normal de l'État, - cela ne fait aucun doute - mais elle est son moyen spécifique. De nos jours la relation entre État et violence est tout particulièrement intime. Depuis toujours les groupements politiques les plus divers - à commencer par la parentèle - ont tous tenu la violence physique pour le moyen normal du pouvoir. Par contre il faut concevoir l'État contemporain comme une communauté humaine qui, dans les limites d'un territoire déterminé - la notion de territoire [101] étant une de ses caractéristiques - revendique avec succès pour son propre compte le monopole de la violence physique légitime. Ce 
qui est en effet le propre de notre époque, c'est qu'elle n'accorde à tous les autres groupements, ou aux individus, le droit de faire appel à la violence que dans la mesure où l'État le tolère : celui-ci passe donc pour l'unique source du «droit» à la violence. Par conséquent, nous entendrons par politique l'ensemble des efforts que l'on fait en vue de participer au pouvoir ou d'influencer la répartition du pouvoir, soit entre les États, soit entre les divers groupes à l'intérieur d'un même État.

En gros, cette définition correspond à l'usage courant du terme. Lorsqu'on dit d'une question qu'elle est « politique », d'un ministre ou d'un fonctionnaire qu'ils sont « politiques », ou d'une décision qu'elle a été déterminée par la "politique », il faut entendre par là, dans le premier cas que les intérêts de la répartition, de la conservation ou du transfert du pouvoir sont déterminants pour répondre à cette question, dans le second cas que ces mêmes facteurs conditionnent la sphère d'activité du fonctionnaire en question, et dans le dernier cas qu'ils déterminent cette décision. Tout homme qui fait de la politique aspire au pouvoir - soit parce qu'il le considère comme un moyen an service d'autres fins, idéales ou égoïstes, soit qu'il le désire " pour lui-même » en vue de jouir du sentiment de prestige qu'il confère.

Comme tous les groupements politiques qui l'ont précédé historiquement, l'État consiste en un rapport de domination de l'homme sur l'homme fondé sur le moyen de la violence légitime (c'est-à-dire sur la violence qui est considérée comme légitime). L'État ne peut donc exister qu'à la condition que les hommes dominés se soumettent à l'autorité revendiquée chaque fois par les dominateurs. Les questions suivantes se posent alors. Dans quelles conditions se soumettent-ils et pourquoi ? Sur [102] quelles justifications internes et sur quels moyens externes, cette domination s'appuie-t-elle?

Il existe en principe - nous commencerons par là - trois raisons internes qui justifient la domination, et par conséquent il existe trois fondements de la légitimité. Tout d'abord l'autorité de l'« éternel hier ", c'est-à-dire celle des coutumes sanctifiées par leur validité immémoriale et par l'habitude enracinée en l'homme de les respecter. Tel est le « pouvoir traditionnel » que le patriarche ou le seigneur terrien exerçaient autrefois. En second lieu l'autorité fondée sur la grâce personnelle et extraordinaire d'un individu (charisme) ; elle se caractérise par le dévouement tout personnel des sujets à la cause d'un 
homme et par leur confiance en sa seule personne en tant qu'elle se singularise par des qualités prodigieuses, par l'héroïsme ou d'autres particularités exemplaires qui font le chef. C'est là le pouvoir « charismatique » que le prophète exerçait, ou - dans le domaine politique - le chef de guerre élu, le souverain plébiscité, le grand démagogue ou le chef d'un parti politique. Il y a enfin l'autorité qui s'impose en vertu de la " légalité », en vertu de la croyance en la validité d'un statut légal et d'une a compétence » positive fondée sur des règles établies rationnellement, en d'autres termes l'autorité fondée sur l'obéissance qui s'acquitte des obligations conformes au statut établi. C'est là le pouvoir tel que l'exerce le «serviteur de l'État» moderne, ainsi que tous les détenteurs du pouvoir qui s'en rapprochent sous ce rapport.

Il va de soi que dans la réalité des motifs extrêmement puissants, commandés par la peur ou par l'espoir, conditionnent l'obéissance des sujets - soit la peur d'une vengeance des puissances magiques ou des détenteurs du pouvoir, soit l'espoir en une récompense ici-bas ou dans l'autre inonde; mais elle peut également être conditionnée par d'autres intérêts très variés. Nous y reviendrons tout à l'heure. Quoi qu'il en soit, chaque fois que [103] l'on s'interroge sur les fondements qui « légitiment » l'obéissance, on rencontre toujours sans contredit ces trois formes « pures » que nous venons d'indiquer.

Ces représentations ainsi que leur justification interne sont également d'une très grande importance pour la structure de la domination. Il est certain que dans la réalité on ne rencontre que très rarement ces types purs. Cependant nous ne pouvons pas exposer aujourd'hui dans le détail les variétés, les transitions et les combinaisons extrêmement embrouillées de ces types; pareille étude entre dans le cadre d'une « théorie générale de l'État».

Pour le moment nous porterons particulièrement notre attention sur le deuxième type de légitimité, à savoir le pouvoir issu de la soumission des sujets au «charisme » purement personnel du « chef ». En effet, ce type nous conduit à la source de l'idée de vocation, où nous retrouvons ses traits les plus caractéristiques. Si certains s'abandonnent au charisme du prophète, du chef en temps de guerre, du très grand démagogue au. sein de l'ecclesia ou du Parlement, cela signifie que ces derniers passent pour être intérieurement " appelés » au rôle de conducteur d'hommes et qu'on leur obéit non pas en vertu d'une 
coutume ou d'une loi, niais parce qu'on a foi en eux. Certes, s'il est plus qu'un petit parvenu présomptueux du moment, il vit pour sa chose, il cherche à accomplir son œuvre. Par contre c'est uniquement à sa personne et à ses qualités personnelles que s'adresse le dévouement des siens, qu'ils soient des disciples des fidèles ou encore des militants liés à leur chef. L'histoire nous montre que l'on rencontre des chefs charismatiques dans tous les domaines et à toutes les époques historiques. Ils ont cependant surgi sous l'aspect de deux figures essentielles, celle du magicien et du prophète d'une part et celle du chef de guerre élu, du chef de bande et du condottiere de l'autre. Mais ce qui est [104] propre à l'Occident - et cela nous intéresse plus spécialement - c'est la figure du libre «démagogue ». Celui-ci n'a triomphé qu'en Occident, au sein des cités indépendantes, particulièrement dans les pays de civilisation méditerranéenne. De nos jours ce même type se présente sous l'aspect du " chef d'un parti parlementaire "); on ne le rencontre de même qu'en Occident qui est la terre des États constitutionnels.

Ce genre d'hommes politiques par « vocation », au sens propre du terme, ne constitue évidemment dans aucun pays la seule figure déterminante de l'entreprise politique et de la lutte pour le pouvoir. Le facteur décisif consiste - plutôt dans la nature des moyens dont les hommes politiques disposent. De quelle manière les forces politiques dominantes s'y prennent-elles pour affirmer leur autorité ? Cette question concerne toutes les espèces de domination et par conséquent elle vaut également pour toutes les formes de domination politique, qu'elle soit traditionaliste, légaliste ou charismatique.

Toute entreprise de domination [Herrschaftsbetrieb] qui réclame une continuité administrative exige d'une part que l'activité des sujets s'oriente en fonction de l'obéissance due aux maîtres qui prétendent être les détenteurs de la force légitime et d'autre part que, moyennant cette obéissance, elle puisse disposer des biens matériels qui sont, le cas échéant, nécessaires pour appliquer la force physique. En d'autres 
termes elle a besoin d'une part d'un état-major administratif et d'autre part de moyens matériels de gestion.

L'état-major administratif qui figure l'aspect extérieur de l'entreprise de domination politique, comme d'ailleurs de n'importe quelle autre entreprise, n'est évidemment pas tenu à l'obéissance envers le détenteur du pouvoir par les seules conceptions de légitimité dont il a été question plus [105] haut. Son obéissance se fonde plutôt sur deux sortes de motifs qui en appellent à l'intérêt personnel : la rétribution matérielle et l'honneur social. Le fief des vaisseaux, les prébendes des ministeriales, les émoluments des actuels serviteurs de l'État d'une part, l'honneur du chevalier, les privilèges des ordres, la dignité du fonctionnaire d'autre part, constituent leur récompense ; et la crainte de perdre tous ces avantages est la raison décisive de la solidarité qui lie l'état-major administratif au détenteur du pouvoir. Il en va de même dans les cas de domination charismatique : celle-ci procure aux soldats fidèles la gloire guerrière et le butin, aux partisans du démagogue les « dépouilles à, c'est-à-dire l'exploitation des administrés grâce au monopole des charges, aux petits profits de l'activité politique et aux récompenses de la vanité.

La stabilité de toute domination par la violence a besoin, tout comme une entreprise économique, de certains biens matériels. On peut, de ce point de vue, classer les administrations en deux catégories. La première obéit au principe suivant : l'état-major, les fonctionnaires ou autres magistrats sur l'obéissance desquels le détenteur du pouvoir doit pouvoir compter, sont eux-mêmes propriétaires des moyens de gestion; ceux-ci peuvent consister en moyens financiers, bâtiments, matériel de guerre, parcs de voiture, chevaux, etc. L'autre catégorie obéit au principe opposé : l'état-major est " coupe » des moyens de gestion dans le même sens où de nos jours l'employé et le prolétaire sont a coupes » des moyens de production matériels dans l'entreprise capitaliste. Il s'agit donc toujours de savoir si le détenteur du pouvoir détient l'administration en régie propre, s'il l'organise luimême et confie la gérance à des serviteurs attaches à sa personne, à des employés qu'il a embauches ou à des favoris et des familiers qui ne sont pas propriétaires, c'est-à-dire qui ne sont pas possesseurs de plein droit des moyens de gestion, ou si au contraire [106] l'administration est entre les mains de personnes économiquement indépen- 
dantes du pouvoir. On retrouve cette différence dans toutes les administrations qui nous sont connues.

Nous donnerons au groupement politique dans lequel les moyens matériels de gestion sont en totalité ou en partie propriété personnelle de l'état-major administratif le nom de, groupement organisé « selon le principe des ordres » [ou des états, ständisch]. Dans la société féodale par exemple, le vassal faisait face, par ses propres moyens, aux dépenses de l'administration et de la justice dans le territoire qui lui avait été confié et il s'équipait et s'approvisionnait lui-même pour la guerre. Les vassaux qui lui étaient subordonnés faisaient de même. Cette situation aboutissait à certaines conséquences dans l'exercice du pouvoir du suzerain puisque sa puissance ne se fondait que sur le serment personnel d'allégeance et sur la particularité que la « légitimité » de la possession d'un fief et l'honneur social du vassal dérivaient du suzerain.

Cependant on trouve également partout, même dans les formations politiques les plus anciennes, la régie personnelle du chef. Celui-ci cherche à devenir le maître de l'administration en la confiant à des subordonnés attachés à sa personne, à des esclaves, à des domestiques, à des commis, à des favoris ou encore à des prébendés qu'il attire par des dotations en nature ou en espèces prélevées sur ses réserves. Il fait face aux dépenses administratives par des prélèvements sur sa fortune personnelle ou bien en distribuant les revenus de son patrimoine et il crée une armée dépendant personnellement de sa seule autorité puisqu'elle est équipée et approvisionnée dans ses greniers, dans ses magasins et dans son arsenal. Dans le premier cas, celui d'un groupement structuré en "états », le souverain ne gouverne qu'avec l'aide d'une aristocratie indépendante et partage de ce fait avec elle le pouvoir. Dans le second cas il s'appuie soit sur des gens de sa maison, soit sur des plébéiens, [107] c'est-à-dire sur des couches sociales sans fortune et dépourvues de tout honneur social propre. Par conséquent ces derniers dépendent entièrement de lui du point de vue matériel, mais surtout ils ne sont soutenus par aucune sorte de pouvoir capable de concurrencer celui du souverain. Toutes les espèces de pouvoir patriarcal et patrimonial aussi bien que le despotisme d'un sultan et les États à structure bureaucratique appartiennent à ce dernier type, - j'insiste tout particulièrement sur l'État 
bureaucratique parce qu'il caractérise au mieux le développement rationnel de l'État moderne.

Partout le développement de l'État moderne a pour point de départ la volonté du prince d'exproprier les puissances « privées » indépendantes qui, à côté de lui, détiennent un pouvoir administratif, c'est-àdire tous ceux qui sont propriétaires de moyens de gestion, de moyens militaires, de moyens financiers et de foutes les sortes de biens susceptibles d'être utilisés politiquement. Ce processus s'accomplit en parfait parallèle avec le développement de l'entreprise capitaliste expropriant petit à petit les producteurs indépendants. Et finalement on voit que dans l'État moderne le pouvoir qui dispose de la totalité des moyens de gestion politiques tend à se ramasser en une seule main ; aucun des fonctionnaires ne reste plus propriétaire personnel de l'argent qu'il dépense ou des bâtiments, des stocks et des machines de guerre qu'il contrôle. L'État contemporain et cela est important sur le plan des concepts a donc entièrement réussi à " couper » la direction administrative, les fonctionnaires et les travailleurs de l'administration des moyens de gestion. C'est alors que l'on vit apparaître un processus tout nouveau qui se déroule actuellement sous nos yeux et qui essaye d'exproprier l'expropriateur des moyens politiques et du pouvoir politique. Telle semble du moins être l'œuvre de la révolution [allemande de 1918] dans la mesure où [108] des chefs nouveaux se sont substitués aux autorités établies, où ils se sont emparés par usurpation ou élection du pouvoir qui contrôle l'ensemble de l'administration et l'appareil des biens matériels, et où ils font dériver peu importe avec quel droit - leur légitimité de la volonté des gouvernés. Mais on peut se demander si ce premier succès - au moins apparent - permettra à la révolution de réaliser l'expropriation de l'appareil économique du capitalisme dont l'activité s'oriente, par essence, selon des lois totalement différentes de celles de l'administration politique. Il ne nous appartient pas de prendre aujourd'hui position sur ce point. Je me contenterai de retenir pour mon propos cette constatation d'ordre purement conceptuel : l'État moderne est un groupement de domination de caractère institutionnel qui a cherché (avec succès) à monopoliser, dans les limites d'un territoire, la violence physique légitime comme moyen de domination et qui, dans ce but, a réuni dans les mains des dirigeants les moyens matériels de gestion. Ce qui veut dire qu'il en a exproprié tous les fonctionnaires qui, suivant le prin- 
cipe des « états », en disposaient autrefois de leur propre droit et qu'il s'est substitué à eux, même au sommet de la hiérarchie.

Mais au cours de ce processus d'expropriation politique qui s'est affirmé avec plus ou moins de succès dans tous les pays de la terre, on vit apparaître une nouvelle sorte $\mathrm{d}^{\prime}$ « hommes politiques professionnels ». Il s'agit en l'occurrence d'une nouvelle catégorie permettant de définir le deuxième sens de cette expression. On les vit tout d'abord se mettre au service des princes. Ils n'avaient pas l'ambition des chefs charismatiques et ne cherchaient pas à devenir des maîtres, mais ils entraient dans la lutte politique pour se mettre à la disposition d'un prince, la gestion de ses intérêts politiques leur fournissait leur gagne-pain et le contenu moral de leur vie. Ce n'est [109] de nouveau qu'en Occident que nous rencontrons cette nouvelle catégorie d'hommes politiques professionnels bien qu'on les trouve également au service d'autres puissances que celle des seuls princes. Cependant ils furent autrefois l'instrument le plus important du pouvoir des princes et de l'expropriation politique qui se faisait à leur profit.

Avant d'entrer dans les détails, essayons de comprendre clairement, sans équivoque et sous tous ses aspects, la signification de l'apparition de cette nouvelle sorte $\mathrm{d}^{\prime}$ « hommes politiques professionnels ». On peut faire de la politique de plusieurs manières - ce qui veut dire que l'on peut influer de plusieurs façons la répartition du pouvoir entre les formations politiques ou à l'intérieur de chacune d'elles. On peut faire de la politique d'une manière " occasionnelle », mais on peut également faire de l'activité politique une profession secondaire ou une profession principale, tout comme dans l'activité économique. Nous faisons tous «occasionnellement» de la politique lorsque nous mettons notre bulletin de vote dans l'urne ou lorsque nous exprimons pareillement notre volonté, par exemple en manifestant notre désapprobation ou notre accord au cours d'une réunion «politique» ou enfin lorsque nous prononçons un discours «politique », etc. D'ailleurs, pour de très nombreuses personnes le contact avec la politique se réduit à ce genre de manifestations. D'autres personnes font de l'activité politique leur profession " secondaire». Il en est ainsi de tous ceux qui jouent le rôle d'hommes de confiance ou de membres des partis politiques et qui, en règle générale, n'exercent cette activité qu'en cas de besoin sans cependant en faire a leur vie ", ni matériellement ni moralement. Il en est de même des membres des 
Conseils d'État ou des autres organismes consultatifs qui n'entrent en fonction que lorsqu'on fait appel à eux. Il en est de même également d'assez [110] nombreux parlementaires qui n'ont d'activité politique que pendant la durée des sessions. On rencontrait très souvent, autrefois, ce genre d'hommes politiques dans l'organisation par « ordres » de l'ancien régime. Nous désignons par « ordres » les propriétaires, par droit personnel, des moyens de gestion matériels d'ordre administratif et militaire ou les bénéficiaires de privilèges personnels. Or une grande partie des membres de ces a ordres » était loin de consacrer en totalité leur vie à la politique, ou même seulement leurs préférences ; ils ne faisaient de la politique qu'occasionnellement. Ils ne voyaient dans leurs prérogatives qu'un moyen pour s'assurer des rentes ou encore un profit personnel. Aussi ne déployaient-ils une activité politique à l'intérieur de leur groupement que si leur suzerain ou leurs pairs le leur demandaient expressément. Il en était de même d'une importante fraction des forces auxiliaires que le prince attirait à son service pour en faire un instrument de la lutte qu'il menait pour constituer une organisation politique dévouée à sa personne. Les « conseillers privés » [Raie von Haus aus] faisaient partie de cette catégorie et, en remontant plus loin, une grande partie des conseillers qui siégeaient dans la curia ou autres organismes consultatifs du prince. Évidemment ces auxiliaires, qui ne faisaient qu'occasionnellement de la politique ou qui ne voyaient en elle qu'une activité secondaire, étaient loin de faire l'affaire du prince. Il ne restait donc à celuici d'autre moyen que de chercher à s'adjoindre un corps de collaborateurs entièrement et exclusivement dévoués à sa personne et qui fassent de l'activité politique leur occupation principale,. Naturellement la structure de l'organisation politique de la dynastie naissante en même temps que toute la physionomie de la civilisation examinée dépendra chaque fois, pour une grande part, de la couche sociale dans laquelle le prince recrutera ses agents.

[111]

Il en sera de même, à plus forte raison, des groupements politiques qui, après abolition complète ou limitation considérable du pouvoir seigneurial, se constitueront politiquement en communes «libres " - libres non dans le sens d'une libération de toute domination par la violence, mais dans celui de l'absence d'un pouvoir seigneurial légitimé par la tradition, le plus souvent consacré par la reli- 
gion et considéré comme unique source de toute autorité. Ces communes ont en historiquement, elles aussi, leur berceau en Occident seulement, sous la forme primitive de la ville constituée en groupement politique, telle qu'on la vit apparaitre pour la première fois dans l'aire de la civilisation méditerranéenne. Essayons maintenant de voir sous quels aspects se sont présentées toutes ces catégories qui faisaient de la politique leur « profession principale ».

Il y a deux façons de faire de la politique. On bien on vit «pour » la politique, ou bien «de » la politique. Cette opposition n'a absolument rien d'exclusif. Bien plutôt on fait en règle générale les deux à la fois, idéellement certes, mais aussi la plupart du temps matériellement. Celui qui vit «pour» la politique fait d'elle, dans le sens le plus profond du terme, le « but de sa vie », soit parce qu'il trouve un moyen de jouissance dans la simple possession du pouvoir, soit parce que cette activité lui permet de trouver son équilibre interne et d'exprimer sa valeur personnelle en se mettant au service d'une " cause » qui donne un sens à sa vie. C'est en ce sens profond que tout homme sérieux qui vit pour une cause vit également d'elle. Notre distinction a donc pour base un aspect extrêmement important de la condition de l'homme politique, à savoir l'aspect économique. Nous dirons donc que celui qui voit dans la politique une source [112] permanente de revenus «vit de la politique » et que, dans le cas contraire, il vit " pour » elle. Sous le régime fondé sur la propriété privée il est nécessaire que soient réunies certaines conditions, triviales si vous voulez, afin qu'un homme puisse vivre " pour» la politique en ce sens. L'homme politique doit, dans des conditions normales, être économiquement indépendant des revenus que l'activité politique pourrait lui procurer. Cela vent dire qu'il est indispensable de posséder une fortune personnelle on d'occuper une situation sociale privée susceptible d'assurer des revenus suffisants. Il en est du moins ainsi dans les conditions normales, car les partisans du chef de guerre s'inquiètent tout aussi peu des conditions d'une économie normale que les compagnons de l'agitateur révolutionnaire. Dans les deux cas on ne vit que de butin, de vols, de confiscations, du cours forcé de bons de paie- 
ment sans valeur, - car tout cela est au fond la même chose. Ces situations sont cependant nécessairement exceptionnelles; dans la vie économique de tous les jours, la fortune personnelle procure seule l'indépendance économique. L'homme politique doit en outre être « économiquement disponible » [abkömmlich], ce qui veut dire que l'acquisition de revenus ne l'oblige pas à consacrer constamment et personnellement, en tout on partie, toute sa puissance de travail et de pensée à sa subsistance. Or celui qui est le plus, " disponible » en ce sens, c'est le rentier, par conséquent la personne qui perçoit des revenus sans aucun travail, soit qu'à l'instar des seigneurs d'autrefois ou des grands propriétaires fonciers et de la haute noblesse d'aujourd'hui il les tire de rentes foncières - dans l'Antiquité et au Moyen Age on les tirait également de rentes constituées par les esclaves ou les serfs -, soit qu'il les tire de titres ou d'autres sources analogues. Ni l'ouvrier, ni non plus - cela doit être particulièrement signalé - l'entrepreneur moderne, et surtout le [113] grand entrepreneur, ne sont disponibles en ce sens. L'entrepreneur surtout est lié à son entreprise et il n'est donc pas disponible, l'entrepreneur industriel encore beaucoup moins que l'entrepreneur agricole, étant donné le caractère saisonnier de l'agriculture. La plupart du temps il lui est difficile de se faire remplacer, même temporairement. De même le médecin n'est pas non plus disponible et il l'est d'autant moins qu'il est plus éminent et qu'on vient le consulter plus fréquemment. Pour des raisons de pure technique professionnelle les difficultés sont déjà moindres dans le cas de l'avocat, ce qui explique qu'il a joué comme homme politique professionnel un rôle incomparablement plus grand et souvent directement prépondérant. Mais il n'est pas nécessaire de développer plus longuement cette casuistique; tirons plutôt au clair quelques-unes des conséquences de ce que nous venons d'exposer.

Le fait qu'un État ou un parti sont dirigés par des hommes qui, dans le sens économique du mot, vivent exclusivement pour la politique et non de la politique signifie nécessairement que les couches dirigeantes se recrutent de façon "ploutocratique ». En disant cela tous ne cherchons nullement à faire accroire que la direction ploutocratique ne profite pas de sa situation dominante pour vivre également « de » la politique et pour exploiter sa position politique au profit de ses intérêts économiques. Cela va sans dire. Il n'existe pas de couches dirigeantes qui ne l'aient fait d'une façon ou d'une autre. 
Notre propos signifie simplement que les hommes politiques professionnels ne sont pas toujours directement contraints de réclamer un dédommagement pour leurs services politiques alors que l'individu dépourvu de fortune est obligé de prendre cet aspect en considération. D'un autre côté nous ne cherchons pas non plus à insinuer que les hommes politiques sans fortune n'auraient d'autre souci [114] au cours de leur activité politique que de se procurer, seulement ou même surtout, des avantages économiques privés et qu'ils ne pensent on ne songent pas en premier lieu à la cause qui est la leur. Rien ne serait plus faux. On sait par expérience que le souci de sa " sécurité » économique est précisément - de façon consciente ou non - le point cardinal dans l'orientation de la vie d'un homme qui possède déjà une fortune. L'idéalisme politique que n'arrête aucune considération ni aucun principe se rencontre, sinon exclusivement du moins principalement, chez les individus qui, à cause de leur pauvreté, restent en marge des couches sociales qui ont intérêt à conserver l'ordre économique d'une société donnée. Cela se voit surtout pendant les périodes exceptionnelles, donc révolutionnaires. Tout ce que nous voulons faire ressortir, c'est le fait suivant : le recrutement non ploutocratique du personnel politique, qu'il s'agisse des chefs ou des partisans, est lié à cette condition évidente que l'entreprise politique devra leur procurer des revenus réguliers et assurés. Il n'existe donc jamais que deux possibilités. Ou bien l'on exerce « honorifiquement» l'activité politique, et dans ce cas elle ne peut être menée que par des personnes qui sont, comme on dit, " indépendantes », c'est-à-dire par des personnes qui jouissent d'une fortune personnelle, avant tout par des rentiers. Ou bien l'on ouvre les avenues du pouvoir à des personnes salis fortune et, dans ce cas, l'activité politique exige rémunération. L'homme politique professionnel qui vit « de » la politique peut n'être qu'un pur « prébendier » ou bien encore un « fonctionnaire » rémunéré. En d'autres termes il peut percevoir ses revenus, soit sous la forme d'honoraires ou d'émoluments pour des services déterminés - les potsde-vin n'étant qu'une forme dénaturée, irrégulière et formellement illégale de cette sorte de revenus -, soit sous la forme d'une rémunération fixe en nature [115] ou en espèces, soit sous les deux formes à la fois. Il peut donc revêtir le caractère d'un "entrepreneur» à la manière du condottiere, du fermier et de l'acheteur de charges d'autrefois on encore du boss américain qui considère ses dépenses comme un placement de capitaux qu'il transforme en source de revenus par l'ex- 
ploitation de son influence politique ; ou bien il peut simplement toucher un traitement fixe à la manière du rédacteur ou du secrétaire d'un parti ou du ministre et du fonctionnaire politique modernes. Le dédommagement typique que les princes, les conquérants victorieux ou les chefs triomphants du parti accordaient autrefois à leurs partisans consistait en fiefs, donations de terre, prébendes de toutes sortes et, avec le développement de l'économie financière, plus particulièrement en gratifications. De nos jours, ce sont des postes de toutes sortes dans les partis, dans les journaux, dans les coopératives, dans les caisses de sécurité sociale, dans les municipalités ou dans l'administration de l'État que les chefs de parti distribuent à leurs partisans pour leurs bons et loyaux services. Toutes les luttes partisanes ne sont donc pas uniquement des luttes pour des buts objectifs, mais elles sont aussi et surtout des rivalités pour contrôler la distribution des emplois.

Toutes les luttes entre les tendances particularistes et les tendances centralistes en Allemagne tournent elles aussi, et même surtout, autour de cette question. Quelles seront les puissances qui contrôleront la distribution des emplois, celles de Berlin ou bien au contraire celles de Munich, de Karlsruhe ou de Dresde ? Les partis s'irritent beaucoup plus des passe-droits dans la distribution des postes que des entorses à leur programme. En France, un mouvement préfectoral, fondé sur les forces respectives des partis politiques, a toujours été considéré comme un bouleversement beaucoup plus important qu'une modification dans le programme gouvernemental, et en fait il [116] suscitait beaucoup plus de bruit dans le pays puisque le programme gouvernemental n'avait en général d'autre signification que verbale. Nombre de partis politiques, notamment ceux d'Amérique, sont devenus, depuis la disparition des vieilles divergences sur l'interprétation de la Constitution, des organisations qui ne s'occupent que de la chasse aux emplois et qui modifient leur programme concret en fonction des voix à capter. En Espagne, du moins jusqu'à ces dernières années, les deux grands partis se succédaient au pouvoir selon le principe d'une alternance consentie, sous la couverture d'élections " préfabriquées » en haut lieu, pour permettre aux partisans de ces deux formations de profiter tour à tour des avantages que procurent les emplois administratifs. Dans les territoires des anciennes colonies espagnoles, les soi-disant «élections » et les soi-disant « révolutions » 
n'ont d'autre but que celui de s'emparer de l'assiette au beurre où les vainqueurs espèrent trouver pitance. En Suisse les partis se répartissent pacifiquement entre eux les emplois suivant le principe de la répartition proportionnelle. D'ailleurs, même en Allemagne, un certain nombre de projets de constitution dits « révolutionnaires », par exemple le premier projet élaboré dans le pays de Bade, se proposent d'étendre le système suisse à la répartition des postes ministériels et considèrent par conséquent l'État et les emplois administratifs simplement comme des institutions destinées à procurer uniquement des prébendes. Ce fut sur tout le parti du Centrum qui s'enthousiasma pour les projets de ce genre et, dans le pays de Bade, il inscrivit même à son programme l'application du principe de la répartition proportionnelle des emplois selon les confessions religieuses, sans se soucier de la capacité politique des futurs dirigeants. La même tendance apparut dans tous les autres partis à la suite de l'augmentation croissante du nombre des postes administratifs, conséquence [117] de la bureaucratisation généralisée, mais aussi à cause de l'ambition croissante des citoyens attirés par la sinécure d'une situation administrative devenue aujourd'hui une sorte d'assurance spécifique pour l'avenir. Ainsi, les partis apparaissent de plus en plus aux yeux de leurs adhérents comme une sorte de tremplin qui leur permettra $>$ d'atteindre cette fin essentielle : assurer l'avenir.

À cette tendance s'oppose cependant le développement de la fonction publique moderne qui exige de nos jours un corps de travailleurs intellectuels spécialisés, hautement qualifiés, préparés à leur tâche professionnelle par une formation de plusieurs années et animés par un honneur corporatif très développé sur le chapitre de l'intégrité. Si ce sentiment de l'honneur n'existait pas chez les fonctionnaires, nous serions menacés d'une effroyable corruption et nous n'échapperions pas à la domination des cuistres. En même temps il y aurait grand péril pour le simple rendement technique de l'appareil d'État dont l'importance économique s'accroît constamment et qui ne cessera de croître encore, surtout si l'on considère les tendances actuel-les à la socialisation. Même aux États-Unis où l'on ignorait autrefois le fonctionnaire de carrière et où le dilettantisme administratif des politiciens marrons permettait de remplacer plusieurs centaines de milliers de fonctionnaires, jusques et y compris les postes de facteurs, en fonction des aléas de l'élection présidentielle, l'ancien type de recrute- 
ment est depuis longtemps battu en brèche par le Civil Service Reform. D'impérieuses exigences d'ordre purement technique sont à l'origine de cette évolution. En Europe, la fonction publique, organisée suivant le principe de la division du travail, s'est constituée progressivement au cours d'un développement qui couvre un demi-millénaire. Les villes et les seigneuries italiennes s'engagèrent les premières dans cette voie; dans [118] les monarchies, ce furent les États conquérants normands. Le pas décisif fut accompli à propos de la gestion des finances du prince. Les obstacles qui ont surgi au moment des réformes administratives de l'empereur Max nous permettent de comprendre combien il a été difficile aux fonctionnaires, même sous la pression de l'extrême nécessité et sous la menace turque, de déposséder un souverain de la gestion financière, alors que ce domaine est sans doute celui qui est le moins compatible avec le dilettantisme d'un prince qui, à cette époque encore, n'était avant tout qu'un chevalier. Pour la même raison le développement de la technique militaire imposait la présence d'un officier de carrière et le perfectionnement de la procédure judiciaire exigeait un juriste compétent. Dans ces trois domaines de la finance, de l'armée et de la justice, les fonctionnaires de carrière triomphèrent définitivement, dans les États évolués, au cours du $\mathrm{XVI}^{\mathrm{e}}$ siècle. C'est ainsi que, parallèlement à l'affermissement de l'absolutisme des princes face aux « ordres », s'amorça l'abdication progressive des premiers en faveur des fonctionnaires qui avaient précisé ment contribué à leur victoire sur les seconds.

En même temps que cette ascension des fonctionnaires qualifiés on peut aussi constater - bien que les transitions soient moins perceptibles - une autre évolution du côté des "dirigeants politiques ». Depuis toujours et dans tous les pays du monde il y eut évidemment des conseillers des princes, qui jouirent effectivement auprès d'eux d'une grande autorité. En Orient, la nécessité de décharger autant que possible le sultan de sa responsabilité personnelle afin de pouvoir mieux assurer le succès de son règne a conduit à la création de la figure typique du « grand vizir ». En Occident, à l'époque de Charles Quint qui fut aussi celle de Machiavel - l'influence qu'exerça la lecture passionnée des rapports des ambassadeurs de la République de Venise sur les cercles spécialisés [119] de la diplomatie eut pour résultat de faire de l'activité diplomatique un art de connaisseurs. Les zélateurs de ce nouvel art, pour la plupart formés à l'humanisme, se considé- 
raient comme une catégorie de spécialistes initiés, tout comme les hommes d'État lettrés de la Chine de la basse période, celle de la division du pays en multiples États. Mais ce fut l'évolution politique des régimes vers le constitutionalisme qui fit sentir de façon définitive et urgente la nécessité d'une direction formellement unifiée de l'ensemble de la politique, y compris la politique intérieure, sous l'égide d'un seul homme d'État. Certes, il y eut toujours depuis de fortes personnalités individuelles qui occupèrent la place de conseillers - ou plutôt en fait - celle de guides des princes. Cependant l'organisation des pouvoirs publics avait primitivement emprunté une autre voie que celle que nous venons de signaler, même dans les États les plus évolués. En effet, on vit tout d'abord se constituer un corps administratif suprême de caractère collégial. En théorie, mais de moins en moins fréquemment en pratique, ces organismes siégeaient sous la présidence personnelle du prince qui seul prenait les décisions. Par l'intermédiaire de ce système qui donna naissance à la procédure des avis, des contre-avis et des votes motivés selon le principe de la majorité et en outre grâce au fait qu'à côté des instances suprêmes officielles le souverain faisait appel à des hommes de confiance attachés à sa personne - le « cabinet 》 - par le canal duquel il prenait ses décisions en réponse aux résolutions des Conseils d'État ou des autres instances officielles de cette sorte (peu importe le nom qu'on leur donnait), le prince, qui tombait de plus en plus dans la situation d'un dilettante, crut pouvoir échapper à l'importance irrémédiablement croissante des fonctionnaires spécialisés et qualifiés et garder ainsi en main la direction suprême. Partout on retrouve cette lutte latente entre les fonctionnaires spécialisés et l'autocratie du prince.

[120]

Les choses ne se modifièrent qu'avec l'apparition des Parlements et des aspirations politiques des chefs de partis parlementaires. Bien que les conditions de ce nouveau développement fussent différentes dans chaque pays, elles conduisirent cependant à un résultat apparemment identique. À quelques nuances près, il est vrai. Ainsi, partout où les dynasties réussirent à conserver une puissance réelle - notamment en Allemagne - les intérêts du prince devinrent solidaires de ceux des fonctionnaires contre les prétentions du Parlement et contre ses aspirations au pouvoir. En effet, les fonctionnaires trouvaient un intérêt dans la possibilité, offerte à certains d'entre eux, de briguer les 
postes de l'exécutif, y compris ceux de ministres, qui devenaient du même coup objet d'un avancement de carrière. De son côté le monarque avait intérêt à nommer les ministres à, son gré et à les choisir dans les rangs des fonctionnaires qui lui étaient dévoués. Et les deux parties trouvaient enfin un intérêt commun dans l'unité de la direction Politique qui pouvait ainsi affronter sans aucune division interne, le Parlement : elles avaient donc intérêt à substituer au système collégial un chef de cabinet qui exprimerait l'unité de vues du ministère. En outre pour pouvoir se maintenir formellement à l'écart des rivalités entre les partis et des attaques qu'ils pouvaient diriger contre le gouvernement, le monarque avait besoin d'une personnalité responsable unique, capable de le couvrir, c'est-à-dire un homme qui pouvait donner des explications aux parlementaires, s'opposer à leurs projets ou négocier avec les partis. Tous ces différents intérêts agirent conjointement dans le même sens et aboutirent à l'autorité unifiée d'un ministre-fonctionnaire. Mais le développement de la puissance du Parlement eut des conséquences encore plus grandes dans le sens de l'unification lorsque, comme en Angleterre, le Parlement réussit à supplanter le monarque. Dans ce cas, le « cabinet » ayant à sa tête un dirigeant parlementaire [121] unique, le « leader », prit la forme d'un Comité qui s'appuyait sur la seule puissance possédant une force réelle dans le pays bien qu'elle restât ignorée des lois, à savoir la puissance du parti politique qui détenait chaque fois la majorité au Parlement. Les organismes collégiaux officiels n'étaient donc plus les organes du pouvoir politique dominant, qui était désormais passé aux partis, et par conséquent ils ne pouvaient plus être les détenteurs du gouvernement réel. Pour être en mesure d'affirmer son autorité à l'intérieur et de mener une grande politique étrangère le parti majoritaire avait besoin avant tout d'un organe directeur résolu, composé uniquement des véritables dirigeants du parti, afin de pouvoir traiter confidentiellement les affaires. Cet organe était précisément le cabinet. Mais vis-à-vis du publie et surtout vis-à-vis du public parlementaire il n'y avait qu'un seul chef responsable de toutes les décisions : le chef de cabinet. Ce n'est qu'aux États-Unis et dans les démocraties qu'ils ont influencées qu'on adopta un système totalement différent qui consiste à placer le chef du parti victorieux, élu par le suffrage universel direct, à la tête de l'appareil des fonctionnaires nommés par lui et à n'exiger le consentement du Parlement qu'en matière de budget et de législation. 
L'évolution qui transformait la politique en une « entreprise » exigeait une formation spéciale de ceux qui participent à la lutte pour le pouvoir et qui en appliquent les méthodes, suivant le principe du parti moderne. Elle aboutit à une division des fonctionnaires en deux catégories : d'un côté les fonctionnaires de carrière et de l'autre les fonctionnaires «politiques ». Certes, cette distinction n'est pas radicale, mais elle est cependant suffisamment nette. Les fonctionnaires « politiques » au sens propre du terme sont en règle générale reconnaissables extérieurement au fait qu'on peut les déplacer à volonté ou du moins les «mettre en disponibilité » comme les préfets en France ou les fonctionnaires [122] du même type dans les autres pays. Cette situation est radicalement différente de celle des fonctionnaires de la magistrature qui sont « inamovibles ». En Angleterre on peut classer dans la catégorie des fonctionnaires politiques tous ceux qui, en vertu d'une convention établie, quittent leur poste à la suite d'un changement de la majorité parlementaire et par conséquent d'un changement de cabinet. Il en est habituellement et spécialement ainsi des fonctionnaires qui ont pour tâche de veiller à l'«administration intérieure ", celle-ci étant essentiellement "politique» et consistant avant tout à maintenir "l'ordre » dans le pays, donc à maintenir le rapport de forces existant. En Prusse, à la suite de l'ordonnance de Puttkamer, les fonctionnaires étaient obligés sous menace d'un rappel à l'ordre, de " prendre la défense de la politique du gouvernement » et, tout comme les préfets en France, on les utilisait également comme appareil officiel pour influencer les élections. Cependant dans le système allemand - contrairement à ce qui se passe dans les autres pays - la plupart des fonctionnaires «politiques » restaient soumis à une règle qui valait pour l'ensemble des fonctionnaires, à savoir que l'accès aux fonctions administratives est toujours lié aux diplômes universitaires, à des examens professionnels et à un stage préparatoire. Cette caractéristique spécifique des fonctionnaires modernes ne vaut pas chez nous pour les chefs de l'appareil politique, c'est-à-dire les ministres. Sous l'ancien régime déjà on pouvait devenir en Prusse ministre des cultes et de l'instruction sans avoir jamais fréquenté personnellement un établissement d'enseignement supérieur alors qu'en principe on ne pouvait devenir conseiller référendaire qu'à la condition d'avoir d'abord passé avec succès les examens prescrits. Un chef de service ou un conseiller référendaire spécialisés étaient donc naturellement - par exemple du temps où Althoff était ministre de l'Édu- 
cation en Prusse - infiniment mieux informés que [123] leur patron des véritables problèmes techniques de leur département. Les choses ne se passaient guère autrement en Angleterre. C'est pourquoi le fonctionnaire spécialisé est le personnage le plus puissant en ce qui concerne les affaires courantes. En réalité, une pareille situation n'a rien d'absurde en soi. Le ministre est avant tout le représentant de la constellation politique au pouvoir ; il a donc pour tâche de faire appliquer le programme de la constellation dont il fait partie, de juger en fonction de ce programme les propositions que lui font les fonctionnaires spécialisés ou de donner à ses subordonnés les directives politiques conformes à la ligne de son parti.

Les choses ne se passent pas autrement dans une entreprise privée. Le souverain véritable, à savoir l'assemblée des actionnaires, y est tout aussi dépourvu d'influence dans la gestion de l'affaire qu'un «peuple» dirige par des fonctionnaires spécialisés. Les personnages qui décident de la politique de l'entreprise, c'est-à-dire les membres du « conseil d'administration » dominé par les banques, ne font rien d'autre que donner les directives économiques et désigner les personnes aptes à diriger l'affaire, car elles n'ont elles-mêmes aucune compétence pour la gérer techniquement. Il est évident que, de ce point de vue, la structure actuelle de l'État révolutionnaire qui abandonne la direction de l'administration à de véritables dilettantes, tout simplement parce qu'ils disposent de mitrailleuses, et qui ne voit dans les fonctionnaires spécialisés que de simples agents d'exécution, ne constitue en principe aucune nouveauté. Ce n'est donc point de ce côté-là qu'il faut chercher les causes des difficultés du système actuel, mais ailleurs. Cependant, il n'entre pas dans nos intentions d'en parler aujourd'hui.

Il convient de porter maintenant notre attention sur les traits particuliers des hommes politiques [124] professionnels, aussi bien des chefs que de leurs partisans. Ils se sont modifiés au cours des temps et aujourd'hui encore ils offrent de multiples variétés. 
Ainsi que nous l'avons déjà vu, les « hommes politiques professionnels » apparurent autrefois avec la lutte qui opposa les princes aux « ordres »; ils se mirent tout d'abord au service des premiers. Examinons-en brièvement les principaux types.

Pour lutter contre les ordres le prince s'appuya sur les couches sociales politiquement disponibles qui n'étaient pas intégrées dans un ordre. Appartenaient en premier lien à cette catégorie les clercs, aussi bien dans les Indes occidentales et orientales qu'en Chine, au Japon, en Mongolie lamaïque on dans les pays chrétiens du Moyen Age. Il y avait à cela une raison technique : ils savaient écrire. On eut recours aux brahmanes, aux prêtres bouddhistes, aux lamas ou bien aux évêques et aux prêtres pour faire d'eux des conseillers politiques parce que l'on trouvait chez eux un potentiel administratif capable d'écrire et susceptible d'être utilisé par l'empereur, les princes on le khan, dans la lutte contre l'aristocratie. Le clerc, et tout particulièrement le clerc célibataire, était en effet à l'écart de l'agitation suscitée par les intérêts politiques et économiques normaux de ce temps et surtout il n'était pas tenté, comme le vassal, de vouloir conquérir au détriment de son maître et au profit de ses propres descendants une puissance politique propre. Du fait de sa condition sociale, il était « coupé » des moyens de gestion de l'administration du prince.,

Les lettrés qui avaient reçu une formation humaniste constituèrent la deuxième catégorie. Il fut un temps où l'on apprenait à faire des discours en latin et des poésies en grec en vue de devenir conseiller politique et surtout historiographe politique d'un prince. C'était l'époque de la prime floraison des écoles humanistes et des fondations royales de chaires de "poétique » : époque rapidement [125] révolue chez nous. Elle a eu certes une influence durable sur notre régime scolaire, mais elle n'a pas en, à vrai dire, de conséquences profondes en politique. Il en fut cependant tout autrement en ExtrêmeOrient. Le mandarin chinois est, ou plutôt, a été à l'origine quelque chose d'analogue à l'humaniste de la Renaissance, c'est-à-dire un fin lettré qui a reçu une éducation humaniste du contact des monuments linguistiques du lointain passé. Si vous lisez le journal de Li HoungTchang vous constaterez qu'il tirait encore sa plus grande fierté d'être l'auteur de poésies et d'être un excellent calligraphe. Cette couche sociale de mandarins, nourris de conventions établies sur le modèle de l'antiquité chinoise, a été déterminante pour tout le destin de la Chine. 
Notre destin aurait pu être le même si nos humanistes avaient en, en leur temps, la moindre chance de s'imposer avec le même succès.

La troisième catégorie était formé par la noblesse de cour. Après avoir réussi à déposséder la noblesse du pouvoir politique qu'elle détenait en tant qu'ordre, les souverains l'attirèrent à la cour et l'employèrent dans des fonctions politiques et diplomatiques. La transformation de notre système d'éducation au cours du XVIIe siècle a été en partie déterminée par le fait que les humanistes lettrés cédèrent, auprès des princes, leur place à des hommes politiques professionnels recrutés dans la noblesse de cour.

La quatrième catégorie consiste en une figure spécifiquement anglaise : le patriciat qui comprenait la petite noblesse et les rentiers des villes que l'on désigne par le terme technique de a gentry ». À l'origine, le souverain l'avait attiré à son service pour lutter contre les barons et lui avait confié les postes du self-government, mais avec le temps il tomba de plus en plus lui-même sous la dépendance de cette couche sociale montante. Elle conserva tous les postes de l'administration locale en assumant gratuitement toutes les charges dans [126] l'intérêt de sa propre puissance sociale. Elle a ainsi préservé l'Angleterre de la bureaucratisation qui fut le sort de tous les pays continentaux.

La cinquième catégorie, celle des juristes formés dans les universités, constitue un type particulier à l'Occident et, avant tout, au continent européen dont elle détermina d'une façon dominante toute la structure politique. La formidable influence posthume du droit romain, sous la forme que lui avait donnée l'État bureaucratique romain de la décadence, n'apparaît nulle part plus nettement que dans le fait suivant : la révolution de la chose publique, si l'on entend par là la progression vers une forme étatique rationnelle, a partout été l'œuvre de juristes éclairés. On peut le constater même en Angleterre, bien que les grandes corporations nationales des juristes y aient contrarié la diffusion du droit romain. Dans aucune autre partie du monde on ne trouve une quelconque analogie avec ce phénomène. Les essais d'une pensée juridique rationnelle de l'école hindoue de Mimâmsâ et les efforts des penseurs islamiques pour faire progresser la pensée juridique antique n'ont pu empêcher la contamination de leur pensée juridique rationnelle par des formes de pensée théologiques. Aucun de ces deux courants n'a été capable de rationaliser entièrement la procé- 
dure légale. Pour mener à bonne fin cette tâche il fallut d'abord assurer la transition avec l'ancienne jurisprudence des Romains qui, on le sait, a été le produit d'une structure politique absolument unique, puisqu'elle s'est élevée du rang d'un État-cité à celui d'un empire mondial. Cette œuvre fut d'abord entreprise par les juristes italiens ; il faut citer ensuite l'Usus modernus des pandectistes, les canonistes du haut Moyen Age et enfin les théories du droit naturel de la pensée juridique chrétienne, sécularisée par la suite. Les grands représentants de ce rationalisme juridique ont été les podestats italiens, les légistes français (qui ont trouvé les moyens de droit pour saper le pouvoir des seigneurs au profit [127] du pouvoir royal), les canonistes et les théologiens qui professèrent la théorie du droit naturel dans les conciles, les juristes de cour et les habiles juges des princes du continent, les théoriciens du droit naturel en Hollande et les monarchomaques, les juristes anglais de la Couronne et du Parlement, la noblesse de robe du Parlement de Paris et enfin les avocats de la Révolution française. Sans ce rationalisme juridique on ne pourrait comprendre ni la naissance de l'absolutisme royal ni la grande Révolution. Si vous parcourez les remontrances du Parlement de Paris ou les cahiers de doléances des États généraux depuis le $\mathrm{XVI}^{\mathrm{e}}$ siècle jusqu'en 1789, vous y trouverez partout l'esprit des juristes. Et si vous passez en revue les professions des membres de la Convention lors de la Révolution, vous y trouverez un seul prolétaire - bien qu'il fût élu selon la même loi électorale que ses collègues - et un très petit nombre d'entrepreneurs bourgeois. Par contre vous y trouverez en masse des juristes de toutes sortes sans lesquels il serait absolument impossible de comprendre la mentalité radicale de ces intellectuels, ainsi que leurs projets. Depuis cette époque, l'avocat moderne et la démocratie ont partie liée. D'un autre côté, ce n'est qu'en Occident que l'on trouve la figure de l'avocat dans le sens spécifique d'une couche sociale indépendante, et cela depuis le Moyen Age où ils se sont multipliés à partir de l'« intercesseur » [Fürsprech] de la procédure germanique, sous l'influence d'une rationalisation des procès.

L'importance des avocats dans la politique occidentale depuis l'apparition des partis politiques n'a rien de fortuit. L'entreprise politique dirigée par des partis n'est précisément qu'une entreprise d'intérêts nous verrons tout à l'heure ce qu'il faut entendre par là. Or le métier de l'avocat spécialisé consiste justement dans la défense efficace des 
intérêts de ceux qui s'adressent à lui. En ce domaine - et c'est la conclusion que l'on peut tirer [128] de la supériorité de la propagande ennemie - l'avocat surpasse tout «fonctionnaire». Sans nul doute il peut faire triompher et donc « gagner » techniquement une cause dont les arguments n'ont qu'une faible base logique et qui est par conséquent, logiquement «mauvaise », mais il est aussi le seul à pouvoir faire triompher et donc " gagner » une cause qui se fonde sur des arguments solides et par conséquent « bonne » en ce sens. Il arrive malheureusement trop souvent que le fonctionnaire, en tant qu'homme politique, fasse d'une « bonne » cause du point de vue des arguments une «mauvaise» cause par suite de maladresses techniques. Nous venons d'en faire l'expérience. Dans une mesure de plus en plus grande la politique se fait aujourd'hui en publie et partant elle se fait avec ces moyens que sont les mots parlés et écrits. Or peser l'effet des mots est une tâche qui fait partie au premier chef de l'activité de l'avocat et non de celle d'un fonctionnaire spécialisé qui n'est pas un démagogue et qui, par définition, ne peut l'être. Si par malheur il essaie de jouer ce rôle, il ne peut d'ordinaire le faire que d'une piètre manière.

Le véritable fonctionnaire - et cette remarque est décisive pour juger notre ancien régime - ne doit pas faire de politique, justement en vertu de sa vocation : il doit administrer, avant tout de façon non partisane. Cet impératif vaut également pour les soi-disant fonctionnaires «politiques », du moins officiellement, dans la mesure où la « raison d'État », c'est-à-dire les intérêts vitaux de l'ordre établi, n'est pas en jeu. Il doit s'acquitter de sa tâche sine ira et studio, « sans ressentiment et sans parti pris ». Par conséquent il ne doit pas faire ce que l'homme politique, aussi bien le chef que ses partisans, est contraint de faire sans cesse et nécessairement, à savoir combattre. En effet prendre parti, lutter, se passionner - ira et studium - sont les caractéristiques de l'homme politique. Et avant tout du chef politique. L'activité [129] de ce dernier est subordonnée à un principe de responsabilité totalement étranger, voire même opposé, à celui du fonctionnaire. L'honneur du fonctionnaire consiste dans son habileté à exécuter consciencieusement un ordre sous la responsabilité de l'autorité supérieure, même si - au mépris de son propre avis - elle s'obstine à suivre une fausse voie. Il doit plutôt exécuter cet ordre comme s'il répondait à ses propres convictions. Sans cette discipline morale, 
dans le sens le plus élevé du terme, et sans cette abnégation, tout l'appareil s'écroulerait. L'honneur du chef politique par contre, celui de l'homme d'État dirigeant, consiste justement dans la responsabilité personnelle exclusive pour tout ce qu'il fait, responsabilité qu'il ne peut ni ne doit répudier ou rejeter sur un autre. Or les fonctionnaires qui ont moralement un sens très élevé de leur métier sont nécessairement de mauvais hommes politiques ; en effet, ils n'ont justement pas à prendre de responsabilités dans le sens politique du terme et par conséquent ils sont, de ce point de vue, des hommes politiques moralement inférieurs. Malheureusement, cette sorte de fonctionnaires occupaient chez nous les postes de direction. C'est cela que nous appelons le « régime des fonctionnaires ». Ce n'est point flétrir l'honneur de la fonction publique allemande que de mettre en évidence ce qu'il $\mathrm{y}$ a de politiquement faux dans ce système lorsqu'on se place au point de vue de l'efficacité politique. Mais revenons aux types de figures politiques.

\section{- 0 -}

Depuis qu'il existe des États constitutionnels et même depuis qu'il existe des démocraties, le "démagogue »a été le type du chef politique en Occident. L'arrière-goût désagréable que nous laisse ce mot ne doit pas nous faire oublier que ce n'est pas Cléon, mais Périclès qui le premier porta ce nom. N'ayant aucune fonction, on plutôt, occupant la [130] seule fonction élective, celle du stratège suprême alors que tous les autres postes de la démocratie antique étaient attribués par tirage au sort - il dirigeait l'ecclesia souveraine du démos athénien. Certes, la démagogie moderne fait également usage du discours et même dans une proportion quantitativement effrayante si l'on songe aux discours électoraux que le candidat moderne est obligé de faire, mais elle fait un usage encore plus constant du mot imprimé. C'est pourquoi le publiciste politique et tout particulièrement le journaliste sont de nos jours les représentants les plus importants de l'espèce.

Il ne nous est absolument pas possible de faire, dans le cadre de cette conférence, même une simple esquisse de la sociologie du jour- 
nalisme moderne. En effet, ce problème constitue, à tout point de vue un chapitre à part. Nous nous contenterons donc de faire les quelques remarques qui sont importantes pour notre sujet. Le journaliste partage le même sort que tous les démagogues ainsi que - du moins sur le continent, contrairement à ce qui se passe en Angleterre et ce qui se passait autrefois en Prusse - l'avocat (et l'artiste) : il échappe à toute classification sociale précise. Il appartient à une sorte de caste de parias que la « société » juge toujours socialement d'après le comportement de ses représentants les plus indignes du point de vue de la moralité. C'est pourquoi l'on colporte couramment les idées les plus saugrenues sur les 'journalistes et sur leur métier. Cependant la plupart des gens ignorent qu'une «œuvre » journalistique réellement bonne exige au moins autant $\mathrm{d}$ ' « intelligence » que n'importe quelle autre œuvre d'intellectuels, et trop souvent l'on oublie qu'il s'agit d'une œuvre à produire sur-le-champ, sur commande, à laquelle il faut donner une efficacité immédiate dans des conditions de création qui sont totalement différentes de celles des autres intellectuels. On soupçonne très rarement que la responsabilité du journaliste est beaucoup plus grande que [131] celle du savant et que le sentiment de responsabilité de tout journaliste honorable n'est en rien inférieur à celui de n'importe quel autre intellectuel - on peut même dire qu'il est plus élevé si l'on se réfère aux constatations que l'on a pu faire durant la dernière guerre. Le discrédit dans lequel est tombé le journalisme s'explique par le fait que nous gardons en mémoire les exploits de certains journalistes dénués de tout sens de leurs responsabilités et qui ont souvent exercé une influence déplorable. Mais personne ne veut admettre que la discrétion du véritable journaliste est en général supérieure à celle des autres personnes. Cela est indéniable. Les tentations incomparablement plus pénibles qui sont liées à l'exercice de ce métier ainsi que les autres conditions de l'activité journalistique moderne impliquent certaines conséquences ; qui ont habitué le public à considérer la presse avec un mélange de mépris et de lâcheté navrante. Mais nous ne pouvons pas examiner ce soir ce qu'il conviendrait de faire en l'occurrence. Ce qui nous intéresse présentement, c'est la question du sort politique réservé aux journalistes: quelles sont leurs chances d'accéder à des postes de direction politique ? jusqu'à présent il n'a existé pour eux de chances favorables que dans le parti social-démocrate, et même à l'intérieur de cette organisation les postes de rédacteurs n'étaient guère, en général, que 
des situations de fonctionnaire et ne constituaient pas un tremplin pour l'accession à une position dirigeante.

Dans les partis bourgeois les chances d'accéder au pouvoir politique par la voie du journalisme se sont plutôt détériorées dans l'ensemble si on les compare à celles de la génération précédente. Naturellement tout homme politique de quelque importance avait besoin de l'influence de la presse et par conséquent de relations avec les milieux de la presse. Mais il était cependant tout à fait exceptionnel contre toute attente - de voir des chefs de parti sortir du rang du journalisme. Il faut en [132] chercher la raison dans la " non-disponibilité » fortement accentuée du journalisme, surtout du journaliste qui n'a aucune fortune personnelle et qui de ce fait n'a d'autres ressources que celles que lui procure sa profession. Cette dépendance est une conséquence du développement énorme, en taille et en pouvoir, de l'entreprise de presse. La nécessité de gagner son pain en rédigeant son article quotidien ou du moins hebdomadaire constitue une sorte de boulet que traîne tout journaliste et je connais parmi eux un certain nombre d'individus qui possédaient le tempérament d'un chef bien qu'ils aient été sans cesse paralysés, matériellement et moralement, dans leur ascension vers le pouvoir. Il est vrai, sous l'ancien régime, les relations de la presse avec les puissances dominantes à l'intérieur de l'État ou des partis ont été aussi désavantageuses que possible pour le niveau du journalisme, mais cela constitue un chapitre à part. Ces relations avaient pris une tout autre allure dans les pays ennemis [Alliés]. Mais même là, et en général dans tous les États modernes, on peut constater, semble-t-il, la règle suivante: le travailleur de la presse perd toujours plus d'influence politique alors que le magnat capitaliste - du type Lord Northcliffe par exemple - ne cesse d'augmenter la sienne.

Bien entendu, les grands consortiums capitalistes de la presse qui s'étaient emparés dans notre pays des journaux dits Moniteur général, publiant des a petites annonces », ont été jusqu'à présent en règle générale, les propagateurs typiques de l'indifférence politique. On s'était rendu compte qu'il n'y avait aucun, profit à tirer d'une politique indépendante et surtout qu'il n'y avait aucun espoir de pouvoir compter sur la bienveillance, commercialement utile, des puissances au pouvoir en s'obstinant dans cette voie. Aussi le système des communiqués a-t-il été largement utilisé par le gouvernement, au cours de la guerre, pour 
essayer d'exercer une influence politique sur la presse et [133] il semble même qu'on veuille pour le moment persévérer dans cette voie. Si l'on peut espérer que la grande presse parviendra à se soustraire à ce mode d'information, il n'en est pas de même en revanche des petites feuilles dont la situation générale est beaucoup plus délicate. Quoi qu'il en soit, la carrière journalistique n'est pas pour le moment, chez nous, une voie normale pour accéder au rang de chef politique (et c'est l'avenir qui nous dira peut-être si elle ne l'est plus ou au contraire si elle ne l'est pas encore), en dépit de l'attrait qu'elle peut au demeurant présenter et de champ d'influence, d'action et de responsabilité qu'elle peut ouvrir à ceux qui veulent s'engager dans cette carrière. Il est difficile de prévoir si l'abandon du principe de l'anonymat réclamé par beaucoup de journalistes - pas par tous il est vrai - est susceptible de modifier la situation. L'expérience que nous avons pu faire dans la presse allemande au cours de la guerre, à propos des journaux qui avaient confié les postes de rédacteur en chef à des écrivains doués d'une grande personnalité et qui s'engageaient expressément sous leur propre nom, nous a malheureusement montré dans quelques cas bien connus que cette méthode n'est peut. être pas aussi bonne qu'on aurait pu le croire pour inculquer un sens élevé de ses responsabilités. Ce furent - sans distinction de partis - les feuilles dites d'information, notoirement les plus infectes, qui s'efforcèrent d'utiliser ce moyen pour augmenter leur tirage et elles réussirent parfaitement en l'occurrence. Les messieurs en question, aussi bien les directeurs de ces publications que les journalistes à sensation, y ont gagné une fortune, mais d'honneur point. Cela ne veut pas dire que l'on doit rejeter le principe de la signature des articles; la question est en effet très complexe et le phénomène que nous venons d'exposer na aucune portée générale. Je constate simplement que cette pratique n'a pas été jusqu'à présent le bon moyen pour former de vrais chefs et des [134] hommes politiques ayant le sens de leurs responsabilités. C'est l'avenir qui nous indiquera comment évoluera cette situation. Quoi qu'il en sait, la carrière journalistique restera néanmoins une des voies les plus importantes de l'activité politique professionnelle. Mais ce n'est pas un chemin pour tout le monde. Surtout pas pour les caractères faibles et moins encore pour les êtres qui ne sont capables de se réaliser que dans une situation sociale sans embûches. Si la vie du jeune intellectuel est déjà exposée au hasard, elle reste pourtant entourée de certaines conventions sociales solides qui la protègent contre les faux 
pas. Mais la vie du journaliste est vraiment livrée, à tous les points de vue, au pur hasard, et cela dans des conditions qui mettent à l'épreuve la sûreté de soi d'une manière qui n'a sans doute pas sa pareille dans aucune autre profession. Les expériences souvent amères de la vie professionnelle sont peut-être l'aspect le moins pénible de ce métier. Car ce sont justement les journalistes de grande notoriété qui sont obligés de faire face à des exigences particulièrement cruelles pour leur personne. En tout cas, ce n'est pas une petite affaire que de fréquenter les salons des puissants de la terre, apparemment sur un pied d'égalité, d'être en général et même souvent adulé, parce que l'on vous craint, et d'avoir en même temps parfaitement conscience que dès que l'on aura franchi le seuil de la porte votre hôte sera peut-être obligé de se justifier devant ses invités de fréquenter les «morveux de la presse ». Et c'est encore bien moins une petite affaire que d'être obligé d'apporter promptement et au surplus avec conviction des vues sur toutes les sortes de questions que le a marché » réclame et sur tous les problèmes imaginables de la vie, et tout cela non seulement sans tomber dans la platitude, mais surtout sans sacrifier à la tendance dépourvue de toute dignité et à ses conséquences implacables qui consiste à se mettre à nu. L'étonnant dans ces circonstances [135] n'est point que de nombreux journalistes aient déraillé ou qu'ils aient déchu du point de vue humain, mais que, en dépit de toutes ces difficultés, la corporation comprenne justement un si grand nombre d'hommes d'authentique valeur et même un nombre de journalistes honnêtes plus important que ne le soupçonnent les profanes.

Si le journaliste est un type d'homme politique professionnel qui a déjà, en un certain sens, un long passé derrière lui, -la figure du fonctionnaire d'un parti politique au contraire n'est apparue qu'au cours des dernières décades et en partie au cours des dernières années. Pour comprendre le développement historique de ce nouveau type il est nécessaire d'examiner d'abord la vie et l'organisation des partis politiques. 
Partout ailleurs que dans les petits cantons ruraux lorsqu'il doit y avoir élection périodique des détenteurs du pouvoir, l'entreprise $[\mathrm{Be}$ trieb] politique est nécessairement une entreprise d'intérêts [Interessenbetrieb]. Cela signifie qu'un nombre relativement restreint d'hommes intéressés au premier chef par la vie politique et désireux de participer au pouvoir recrutent par libre engagement des partisans, se portent eux-mêmes comme candidats aux élections ou y présentent leurs protégés, recueillent les moyens financiers nécessaires et vont à la chasse des suffrages. Il est exclu que l'on puisse organiser pratiquement des élections dans des groupements politiques étendus sans cette sorte d'organisation. Cela veut dire en pratique que les citoyens qui ont le droit de vote se divisent en éléments politiquement actifs et en éléments politiquement passifs. Puisque cette différence a pour base la libre décision de chacun il n'est pas possible de la supprimer, en dépit de toutes les mesures que l'on peut en général envisager, telles que le vote obligatoire, la « représentation des [136] professions » ou n'importe quel autre moyen destiné formellement ou effectivement à faire disparaître cet état de choses et, par ce biais, la domination des politiciens professionnels. L'existence de chefs et de partisans qui en tant qu'éléments actifs cherchent à recruter librement des militants et, par contrecoup, l'existence d'un corps électoral passif constituent des conditions indispensables à la vit de tout parti politique. Mais la structure même des partis politiques peut varier. Les a partis » des villes médiévales, par exemple ceux des Guelfes et des Gibelins, ne se composaient que de clients. Si l'on considère le Statuto della parte Guelfa, si l'on se souvient de certaines dispositions comme la confiscation des biens des Nobili - toutes les familles qui vivaient à la manière des chevaliers et qui pouvaient par conséquent devenir propriétaires d'un fief -, ou encore la suppression du droit d'exercer une fonction on du droit de vote des membres de ces familles, ou enfin si l'on, considère la structure des comités interrégionaux de ce parti, son organisation militaire sévère et les primes qu'il accordait aux dénonciateurs, on ne peut s'empêcher de penser au bolchevisme, à son organisation militaire - et surtout en Russie - à ses organisations de mouchards triés sur le volet, à la déchéance juridique des « bourgeois » avec privation de leurs droits politiques et confiscation de leurs biens, bref à l'élimination des entrepreneurs, des commerçants, des rentiers, des prêtres, des descendants de la famille dynastique et des chefs de l'ancienne police. L'analogie devient encore plus frappante lorsqu'on considère que l'or- 
ganisation militaire du parti guelfe était bâtie sur le principe d'une armée de chevaliers et que les nobles y occupaient presque tous les postes dirigeants ; car les Soviets conservent, ou plutôt rétablissent à nouveau, l'entrepreneur largement rémunéré, le travail à la chaîne, le système Taylor, la discipline dans l'armée et dans l'usine et même [137] jettent des regards vers les capitaux étrangers. En un mot, pour mettre en marche la machine économique et étatique, ils se voient contraints d'adopter tout ce qu'ils avaient condamné comme institutions de la classe 'bourgeoise ; en entre ils réintègrent à nouveau dans leurs fonctions les agents de l'ancienne Ochrana pour en faire les instruments essentiels du pouvoir politique. Dans cette causerie nous n'avons cependant pas à nous occuper de ces sortes d'organisations fondées sur la violence, mais plutôt des hommes politiques professionnels qui cherchent à parvenir au pouvoir grâce à la puissance d'un parti politique qui brigue des voix sur le marché électoral, sans jamais utiliser autre chose que des moyens raisonnables et « pacifiques ».

Si l'on considère maintenant les partis politiques au sens usuel du terme, on constate qu'ils ne comprenaient primitivement, par exemple en Angleterre, que de simples clients de l'aristocratie. Lorsque, pour une raison quelconque, un pair changeait de parti, tous ceux qui dépendaient de lui passaient également dans l'autre camp. Jusqu'à l'époque du Reform Bill [de 183]1 ce n'était pas le roi mais les grandes familles de la noblesse qui jouissaient du patronage de la masse énorme des bourgs électoraux. Les partis de notables qui se sont développés plus tard avec l'ascension politique de la bourgeoisie gardaient encore une structure assez voisine des partis de la noblesse. Les milieux [Kreise] sociaux qui possédaient "fortune et éducation», animés et dirigés par les intellectuels, catégorie propre à l'Occident, se divisèrent en différentes fractions, en partie par intérêt de classe, en partie par tradition familiale, en partie pour des raisons purement idéologiques, et constituèrent des partis politiques dont ils gardèrent la direction. Des membres du clergé, des instituteurs, des professeurs des avocats, des médecins, des pharmaciens, des paysans aisés, des fabricants - et en Angleterre toute la couche [138] sociale qui estimait appartenir à la classe des gentlemen - constituèrent tout d'abord des groupements politiques épisodiques, on tout au plus des clubs politiques locaux ; pendant les périodes troublées on voyait également apparaître sur la scène politique la petite bourgeoisie, et une fois même 
le prolétariat; encore fallait-il que ces dernières couches sociales trouvent un chef qui en règle générale ne sortait pas de leur rang. A ce stade il n'existait pas encore de partis organisés régionalement qui s'appuyassent sur clés groupements permanents à l'intérieur du pays. Il n'existait d'autre cohésion politique que celle que créaient les parlementaires ; cependant les notables locaux jouaient un rôle déterminant dans le choix des candidats. Les programmes étaient constitués, en partie par la profession de foi des candidats, en partie par des emprunts aux résolutions clés réunions de notables ou aux résolutions des fractions parlementaires. Ce n'était jamais qu'accessoirement et à titre purement honorifique qu'un responsable consacrait le cas 'échéant une partie de ses loisirs à la direction d'un club. Dans les localités où il n'en existait pas (ce qui constituait le cas le plus fréquent), l'activité politique était totalement inorganisée, même parmi les rares personnes qui s'intéressaient normalement et de façon continue aux affaires du pays. Seul le journaliste était un homme politique professionnel rémunéré et, à côté des sessions du Parlement, seule l'entreprise de presse constituait une organisation politique ayant quelque continuité. Toutefois les parlementaires et les chefs de partis savaient parfaitement vers quels notables locaux il fallait se tourner le jour où une action politique semblait souhaitable. Dans les grandes villes seulement existaient des sections permanentes des partis avec des cotisations modérées des membres, des rencontres périodiques et des réunions publiques au cours desquelles le député rendait compte de son mandat. Mais de vie politique il n'y en [139] avait réellement que pendant la période électorale.

On ressentit cependant bientôt la nécessité d'une cohésion plus ferme à l'intérieur des partis. De nombreux motifs décidèrent de cette orientation nouvelle : l'intérêt des parlementaires à réaliser des compromis électoraux entre les différentes circonscriptions, le choc que pouvait créer un programme unique et adopté par de larges couches sociales dans le pays et en général l'utilité pour le parti d'une agitation politique unifiée. Néanmoins, même après que l'on eut établi un réseau de sections locales du parti sur les villes de moyenne importance et après que l'on eut installé dans tout le pays des «hommes de confiance » qui restaient en contact permanent avec un membre du groupe parlementaire, la structure de l'appareil du parti ne fut 'Pas modifiée pour autant : elle conserva en principe le caractère d'un groupe- 
ment de notables. En dehors des employés du siège central il n'existait pas encore de fonctionnaires rémunérés, car partout les associations locales étaient dirigées politiquement par des personnes " considérées » en vertu de l'estime dont elles jouissaient par ailleurs dans la population. Les «notables » extra-parlementaires continuaient à exercer leur influence conjointement à la catégorie de notables qui siégeaient au Parlement. La correspondance éditée par le parti fournissait naturellement de façon croissante la nourriture spirituelle de la presse et des réunions publiques locales. Les cotisations régulières des membres devenaient indispensables, dont une partie était destinée à couvrir les frais de l'organisme central. Il y a peu de temps, les organisations politiques allemandes en étaient encore à ce stade. En France elles sont même encore restées en partie au premier stade, celui des liens très instables entre les parlementaires et le petit nombre des notables locaux. Les programmes y sont encore établis dans chaque circonscription par les candidats eux-mêmes ou par leurs protecteurs [140] avant l'ouverture de la campagne électorale, encore que l'on tienne plus ou moins compte, suivant les nécessités locales, des résolutions et des programmes des parlementaires. Ce n'est qu'en partie qu'on y a réussi à ébranler de nos jours ce système. Le nombre des personnes qui, il y a seulement quelques années, faisaient de l'activité politique leur profession principale était donc extrêmement réduit. Il comprenait principalement les députés élus, les quelques employés de l'organisme central, les journalistes et de plus - en France - ceux qui sont à l'« affût d'un poste » et ceux qui, en ayant déjà occupé un, sont dans l'attente d'une situation nouvelle. En général la politique constituait de façon prépondérante une deuxième profession. Le nombre des députés « ministrables » était extrêmement réduit, ainsi d'ailleurs que celui des candidats aux élections, puisque les notables gardaient le contrôle des opérations. Par contre le nombre de ceux qui s'intéressaient indirectement à la politique, surtout du point de vue matériel, était très grand. Toutes les mesures qu'un ministre pouvait prendre et, avant tout, toutes les solutions qu'il pouvait donner aux affaires personnelles tenaient compte de l'influence possible de la décision à prendre sur les prochaines élections. On cherchait en effet à faire aboutir des vœux de toutes sortes par l'entremise du député local; de bon on de mauvais gré -le ministre était donc obligé de lui prêter oreille, surtout s'il appartenait à sa majorité - et pour cette raison chaque député cherchait à en faire partie. Le député détenait le patronage des emplois et en gé- 
néral toutes sortes de patronage dans les affaires de sa circonscription. Et de son côté il soignait ses relations avec les notables locaux pour assurer sa réélection.

À cet état idyllique de la domination des notables et surtout des parlementaires s'opposent de nos jours, de la façon la plus radicale, la structure et l'organisation modernes des partis. Ces [141] nouvelles formations sont des enfants de la démocratie, du suffrage universel, de la nécessité de recruter et d'organiser les masses, de l'évolution des partis vers l'unification de plus en plus rigide au sommet et vers la discipline la plus sévère aux divers échelons. Nous assistons présentement à la décadence de la domination des notables ainsi qu'à celle d'une politique dirigée par les seuls parlementaires. Des individus qui font de l'activité politique leur profession principale, tout en restant en dehors du Parlement, reprennent en main l'entreprise politique. Ce sont ou bien des « entrepreneurs » - à la manière du boss américain ou de l'election agent anglais - ou bien les fonctionnaires des partis, aux appointements fixes. Du point de vue formel nous assistons à une démocratisation accentuée. Ce n'est plus le groupe parlementaire qui établit le programme fixant la ligne de conduite du parti, ce ne sont plus les notables locaux qui décident des candidatures aux élections, mais ce sont des réunions des militants organisés à l'intérieur du parti qui choisissent les candidats et qui d'autre part délèguent certains de leurs membres dans des assemblées de l'instance supérieure, dont il peut exister plusieurs échelons, jusqu'à l'assemblée générale appelée « Congrès du parti ». En fait le pouvoir est maintenant entre les mains des permanents qui sont responsables de la continuité du travail à l'intérieur de leur organisation ou encore entre celles des personnalités qui dominent personnellement ou financièrement l'entreprise, à la manière des mécènes ou des chefs de puissants clubs politiques d'intérêts du genre Tammany Hall. L'élément décisif et nouveau consiste dans le fait que cet immense appareil - la « machine » suivant l'expression caractéristique employée dans le pays anglo-saxons - ou plutôt les responsables de l'organisation peuvent faire échec aux parlementaires et sont à même d'imposer dans une mesure assez considérable leur propre volonté. Cet [142] élément est tout particulièrement important en ce qui concerne le choix des membres de la direction du parti. Seul celui que la machine est prête à suivre, même par-dessus la tête du 
Parlement, pourra devenir le chef. En d'autres termes, l'institution de ces machines signifie l'entrée en jeu de la démocratie plébiscitaire.

Les militants, et surtout les fonctionnaires et les entrepreneurs du parti, attendent naturellement du triomphe de leur chef une compensation personnelle : des postes on d'autres avantages. Ce qui est déterminant, c'est qu'ils les attendent du chef et non pas, ni uniquement, des parlementaires. Ils espèrent avant tout qu'au cours de la campagne électorale l'influence démagogique de la personnalité du chef -leur procurera des voix et des mandats et qu'elle leur ouvrira ainsi les portes du pouvoir, de sorte que les militants auront les plus grandes chances d'obtenir la récompense espérée pour leur dévouement. Du point de vue psychologique, une des forces motrices les plus importantes de tout parti politique consiste dans la satisfaction que l'homme éprouve à travailler avec le dévouement d'un croyant au succès de la cause d'une personnalité et non pas tellement au profit des médiocrités abstraites d'un programme. C'est justement en cela que réside le pouvoir « charismatique » du chef.

Cette forme nouvelle de l'organisation des partis s'est imposée dans une mesure variable dans la plupart des pays, non sans une constante rivalité latente avec les notables locaux et les parlementaires qui luttent pour sauvegarder leur influence. Ce nouveau style apparut pour la première fois au sein d'un parti bourgeois aux États-Unis et au sein d'un parti socialiste en Allemagne. De constants revirements ont évidemment jalonné cette révolution, surtout lorsqu'un, parti se trouvait à un moment donné privé d'un chef unanimement reconnu. Mais même lorsqu'un tel chef existe, il faut toujours faire des concessions de [143] toutes sortes à la vanité et à l'intérêt personnel des notables mêmes du parti. D'autre part la machine peut également tomber sous la coupe des fonctionnaires qui assument régulièrement le travail interne d'organisation. De l'avis d'un certain nombre de cercles de la social-démocratie, ce parti serait actuellement la proie de cette sorte de « bureaucratisation ». Par ailleurs, il ne faut pas oublier que les «fonctionnaires » se soumettent avec une relative facilité à la personne d'un chef démagogique qui fait grande impression. Cela s'explique à la fois par le fait que leurs intérêts matériels et moraux sont intimement fiés au rayonnement et à la puissance qu'ils souhaitent pour leur parti et par le fait que travailler pour l'amour d'un chef procure une plus grande satisfaction intime. Il est par contre infiniment plus difficile de 
devenir un chef dans les organisations où, à côté des fonctionnaires, les «notables » exercent une grande influence à l'intérieur du parti, ainsi qu'on le voit fréquemment dans les partis bourgeois. En, effet les notabilités investissent [au sens psychanalytique] tellement le petit poste de membre du bureau on de la commission administrative qu'il devient « le but même de leur vie ». Leur activité est en général animée par le ressentiment contre le démagogue qui se présente comme l'homo novus, par la conviction de la supériorité de leur expérience de la politique du parti - effectivement il peut arriver qu'elle ait une grande importance - et par le souci idéologique de ne pas rompre avec les anciennes traditions de l'organisation. À l'intérieur du parti ils peuvent d'ailleurs compter sur tous les éléments conservateurs. L'électeur de la campagne, mais aussi celui de la petite bourgeoisie, a les yeux fixés sur les notables dont les noms lui sont familiers depuis toujours. Il se méfie donc de l'ambition d'un inconnu, quitte à lui vouer une fidélité inébranlable le jour où il aura triomphé définitivement. Essayons maintenant d'examiner plus en [144] détail quelques exemples majeurs de cette lutte entre ces deux formes de structure des partis et notamment les progrès accomplis dans le sens de la forme plébiscitaire décrite par Ostrogorski.

\section{- $\mathbf{0}$ -}

Commençons par l'Angleterre. Jusqu'en 1868 l'organisation clés partis avait presque partout l'aspect d'un pur groupement de notables. Les Tories s'appuyaient dans les campagnes sur le clergé anglican et en outre - souvent même - sur l'instituteur et surtout sur les grands propriétaires des différents comtés. Les Whigs de leur côté s'appuyaient le plus souvent sur le prédicateur non conformiste (là où il $\mathrm{y}$ en avait), sur le maître de poste, le forgeron, le tailleur, le cordier, bref les espèces d'artisans qui, parce qu'on avait très souvent l'occasion de bavarder avec eux, pouvaient exercer une influence politique. Dans les villages la coupure entre les partis politiques se faisait en partie pour des raisons économiques, en partie pour des raisons religieuses et en partie simplement en fonction des opinions traditionnellement reçues dans les familles. Cependant les notables restaient partout les détenteurs du pouvoir au sein des organisations politiques. Au-dessus 
de tout cela planaient le Parlement et les partis dirigés par le Cabinet et son « leader». Ce dernier était ou bien le chef du conseil des ministres ou celui de l'opposition. Le leader était assisté d'un homme politique professionnel, qui jouait un rôle extrêmement important à l'intérieur du parti, le « rabatteur» [whip]. C'est lui qui détenait le patronage des emplois, c'est à lui que devaient s'adresser tous ceux qui cherchaient nie situation politique et c'est encore lui qui les distribuait après avoir consulté les députés des différentes circonscriptions électorales. Mais on vit monter lentement dans chaque circonscription une nouvelle catégorie d'hommes politiques professionnels [145] qui n'étaient à l'origine que des agents locaux non rémunérés, à la manière de nos " hommes de confiance » [Vertrauensmänner]. En outre à la suite d'une législation nouvelle sur les élections destinée à en garantir la régularité on vit apparaître dans les circonscriptions un type d'entrepreneur capitaliste, l'election agent. Il de vint un personnage indispensable du fait que cette nouvelle législation avait pour but de contrôler les dépenses électorales et de contrecarrer les puissances d'argent en contraignant le candidat à déclarer les sommes qu'il dépensait au cours de sa campagne. Il en résulta que tout candidat récoltait, outre la fatigue vocale, la satisfaction de débourser une certaine somme d'argent, bien plus même qu'autrefois chez nous. En principe l'election agent demandait au candidat une somme forfaitaire et faisait ainsi le plus souvent une bonne affaire. - La répartition des pouvoirs entre le leader et les notables du parti, aussi bien au Parlement que dans le pays, laissait depuis toujours en Angleterre un très grand avantage au premier puisqu'il fallait lui donner les moyens de faire une bonne politique avec continuité. Toutefois l'influence des notables et des parlementaires restait encore assez considérable.

Voilà comment se présentait à peu près la situation dans l'ancienne organisation des partis. Elle était pour moitié une affaire des notables et pour moitié déjà une affaire d'employés et d'entrepreneurs. À partir de 1868 se développa, en premier lien à Birmingham au cours d'élections locales, le système du caucus. Un pasteur non conformiste, aidé par joseph Chamberlain, lui donna naissance. Le prétexte invoqué fut celui d'une démocratisation du droit de vote. Dans le but d'attirer la masse on crut bon de mettre en mouvement un immense appareil de groupements d'apparence démocratique, de constituer dans chaque quartier de la ville un comité électoral, de maintenir une continuité 
dans l'entreprise et [146] de bureaucratiser rigoureusement l'ensemble : on vit donc croître considérablement le nombre des employés rémunérés par les comités locaux qui groupèrent et organisèrent bientôt environ $10 \%$ des électeurs. Des intermédiaires principaux, choisis par élection mais possédant par la suite le droit de cooptation, devinrent les maîtres de la politique du parti. Les forces agissantes se recrutaient dans les comités locaux, principalement dans les milieux qui s'intéressaient à la politique municipale - celle-ci étant partout et toujours le tremplin des chances matérielles les plus solides. Ce furent également ces forces purement locales qui réunirent en premier lien les moyens financiers nécessaires. Cette nouvelle machine qui échappait entièrement à la direction parlementaire eut bientôt à soutenir des combats avec les puissances qui jusqu'alors détenaient le pouvoir, principalement avec le whip. Cependant, grâce à l'appui des personnalités locales qui y trouvaient leur intérêt, elle sortit victorieuse de cette lutte et son triomphe fut si complet que le whip fut obligé de se soumettre et de pactiser avec elle. Il en résulta une centralisation de la totalité du pouvoir entre les mains de quelques hommes et, en fin de compte, entre les mains de la seule personne qui se trouvait à la tête du parti. En effet tout ce système s'est développé au sein du parti libéral parallèlement à l'ascension politique de Gladstone. Les raisons qui donnèrent si rapidement à la machine la victoire sur les notables furent tout d'abord le côté fascinant de la démagogie de grand style pratiquée par Gladstone, la croyance tenace des masses dans le contenu moral de sa politique et, avant tout, le moralisme du personnage. C'est ainsi qu'apparut sur la scène politique anglaise une espèce de césarisme plébiscitaire, sous les traits du dictateur qui règne sur le champ de bataille électoral. L'issue ne se fit pas attendre. En 1877 le système du caucus entra pour la première fois en action au cours d'élections générales. Le résultat [147] fut impressionnant : Disraeli dut abandonner le pouvoir au moment de ses plus grands succès. Dès 1876 la machine était déjà tellement dévouée, au sens charismatique, à la personne de Gladstone que, lorsque se posa la question de l'Home Rule, tout l'appareil, de haut en bas, ne demanda jamais : sommes-nous objectivement sur le terrain qui est celui de Gladstone ? mais fit tout simplement volte-face sur la simple foi en la parole de Gladstone en disant : nous le suivrons en tout ce qu'il fait - et la machine abandonna même son créateur, Chamberlain. 
La machine avait besoin d'un important personnel. Il y a environ aujourd'hui 2000 personnes qui en Angleterre vivent directement de la politique clés partis. Plus nombreux sont encore ceux qui sont simplement à l'affût d'une situation et ceux qui se montrent actifs en raison d'autres intérêts, particulièrement dans la politique municipale. D'autre part, en plus des espérances économiques les politiciens embrigadés dans le caucus peuvent aussi espérer des satisfactions de vanité. Ils peuvent en effet nourrir (normalement) les plus hantes ambitions comme celles de devenir J. P. ou même M. P. Ces situations sont promises en particulier à tous ceux qui peuvent faire la preuve d'une bonne éducation, c'est-à-dire à ceux qui sont des gentlemen. L'honneur suprême qui attend principalement les grands bailleurs de fonds est le titre de pair - car les finances des partis proviennent dans une proportion d'environ $50 \%$ de contributions de donateurs anonymes.

Quelle a été la conséquence de tout ce système ? Tout simplement que les parlementaires anglais, à l'exception des quelques membres du cabinet (et de quelques originaux) sont généralement réduits à la condition de bêtes à voter, parfaitement disciplinées. Chez nous, au Reichstag on a pris l'habitude, en expédiant à son pupitre sa correspondance privée, de faire au moins semblant qu'on se dépense pour le bien du pays. Mais en [148] Angleterre on n'exige même pas ce minimum - le parlementaire n'a rien d'autre à faire que de voter et de ne pas trahir son parti. Il doit faire acte de présence lorsque le whip l'appelle et exécuter ce que, suivant les circonstances, le chef du cabinet ou le leader de l'opposition ont ordonné. Chaque fois qu'elle est dirigée par un homme énergique la machine du caucus ne manifeste presque aucune réaction, à l'échelon local; elle suit tout simplement la volonté du leader. Ainsi donc, au-dessus du Parlement se dresse le chef qui est, en fait, un dictateur plébiscitaire : il entraîne à sa suite les masses par le canal de la machine. À ses yeux les parlementaires ne sont donc que de simples prébendés qui forment sa clientèle.

Mais comment se fait dans ce système la sélection des chefs ? Et tout d'abord suivant quelles aptitudes les choisit-on ? Outre les qualités de volonté qui sont partout dans le monde déterminantes, c'est naturellement la puissance de la parole démagogique qui est décisive. La manière a changé depuis l'époque de Cobden qui s'adressait à l'entendement et celle de Gladstone qui était un technicien de la formule, apparemment pleine de sens, du «laissez parler les faits » jusqu'à 
l'époque contemporaine où l'on utilise fréquemment, pour mettre en mouvement les masses, des moyens qui la plupart du temps n'ont qu'un caractère purement émotionnel, du genre de ceux qu'adopte l'Armée du Salut. On peut à juste titre appeler cet état de choses une « dictature fondée sur l'exploitation et l'émotivité des masses ». Cependant le système du travail des commissions, très développé dans le Parlement anglais, donne à tout homme qui ambitionne un poste de membre de l'organisation dirigeante la possibilité d'apporter son concours et même l'oblige à suivre cette voie pour réussir. Tous les ministres importants des dernières décennies ont été formés par ces commissions parlementaires qui les ont habitués à un travail positif et efficace. La pratique [149] que l'on acquiert comme rapporteur d'une commission aussi bien que l'habitude de la critique publique des délibérations permettent à cette école une véritable sélection des chefs tout en éliminant l'individu qui n'est rien de plus qu'un vulgaire démagogue.

Telle est la situation en Angleterre. Mais le système du caucus qui y règne n'est jamais qu'une 'forme atténuée de la machinerie politique si on la compare à l'organisation des partis en Amérique où l'on a très vite adopté une forme particulièrement pure du régime plébiscitaire. Selon l'idée de Washington, l'Amérique devait être une communauté gérée par des gentlemen. À cette époque, le gentleman était également, comme en Angleterre, un propriétaire foncier ou un homme qui avait fréquenté un collège. Au départ il en a effectivement été ainsi. $\mathrm{Au}$ moment où les partis se constituèrent, les membres de la Chambre des Représentants avaient la prétention dé devenir des chefs politiques à l'image de ceux d'Angleterre, à l'époque du règne des notables. Aussi l'organisation des partis manquait-elle alors de toute discipline. Cette situation dura jusqu'en 1824. Mais déjà, avant les années 20, on avait vu apparaître la machine des partis dans plusieurs municipalités, qui devinrent ainsi le point de départ de la nouvelle évolution. Mais ce fut l'élection du Président Andrew Jackson, le candidat des fermiers de l'Ouest, qui renversa vraiment la vieille tradition. Les chefs parlementaires cessèrent bientôt, peu après 1840, d'être formellement les , dirigeants des partis, au moment où les grands parlementaires - Calhoun, Webster - se retirèrent de la vie politique parce que le Parlement avait perdu presque tout pouvoir dans le pays face à la machine des partis. Si la «machine» plébiscitaire s'est développée de si bonne 
heure dans ce pays, c'est parce qu'en Amérique et là uniquement le chef de l'exécutif, qui était en même temps - c'est là l'élément important - le [150] maître de la distribution des emplois, était un président élu par plébiscite, et qu'en outre, par suite de la « séparation des pouvoirs », il était presque totalement indépendant du Parlement dans l'exercice de ses fonctions. En effet, au lendemain d'une élection présidentielle, les partisans du candidat victorieux se voyaient offrir en récompense un véritable butin de prébendes et d'emplois. On ne manqua pas de tirer les conséquences de ce spoil system élevé systématiquement par Andrew Jackson au rang de principe.

Que signifie de nos jours pour la formation des partis ce spoil system, c'est-à-dire l'attribution de tous les postes fédéraux aux partisans du candidat victorieux ? Tout simplement que des partis, sans aucune base doctrinale, simples appareils à briguer des postes, s'opposent les uns aux autres et fabriquent pour chaque campagne électorale un programme en fonction des chances électorales car dans ce pays, les programmes varient dans une proportion qui n'a pas sa pareille dans aucun autre État, malgré toutes les analogies. La structure des partis est entièrement subordonnée à la seule lutte électorale qui est de loin la plus importante pour le patronage des emplois : le poste de Président de l'Union et ceux de gouverneur dans les divers États. Les programmes et les noms des candidats sont arrêtés, sans intervention des parlementaires, au cours des «con - ventions nationales » des partis c'est-à-dire au cours des congrès des partis qui sont composés formellement, de façon très démocratique, de délégués des assemblées qui tiennent leur mandat des primaries ou assemblées des militants de base. Déjà au cours de ces primaries on choisit les délégués aux conventions en fonction du nom des candidats au poste de la magistrature suprême de l'Union. C'est pourquoi l'on voit sévir à l'intérieur des partis la lutte la plus acharnée autour de la nomination, puisque le président est le maître d'environ 300000 à 400000 postes de fonctionnaires [151] qu'il distribue lui-même après consultation des sénateurs des différents États. De ce fait, les sénateurs sont des hommes politiques puissants. La Chambre des Représentants par contre est relativement impuissante du point de vue politique puisque le patronage des emplois lui échappe totalement et que les ministres, simples auxiliaires du Président élu directement par la population contre tout le monde et aussi contre le Parlement, peuvent remplir leur fonction in- 
dépendamment de la confiance ou de la défiance des Représentants : encore une conséquence du principe de la « séparation des pouvoirs ».

Le spoil system, étayé par cette séparation des pouvoirs, n'a été techniquement possible en Amérique que parce que la jeunesse de sa civilisation pouvait supporter une gestion de purs dilettantes. En effet, le fait que 300000 à 400000 militants d'un parti ne pouvaient alléguer pour toute qualification que les bons et loyaux services rendus à leur parti a conduit à la longue à de grandes difficultés, à une corruption et à un gaspillage sans pareils que seul un pays aux chances économiques illimitées pouvait tolérer.

La figure d'homme politique qui monta à la surface grâce à ce système de la machine plébiscitaire fut celle du boss. Qu'est-ce qu'un boss? C'est un entrepreneur politique capitaliste, pourvoyeur de voix électorales pour son profit et à ses risques et périls. À l'origine il est avocat, tenancier de bar ou propriétaire d'établissements de ce genre ou encore créancier, c'est-à-dire qu'il fait un métier qui lui donne les possibilités de poser les premiers jalons et qui lui permet d'étendre ses filets jusqu'à contrôler un certain nombre de voix. Parvenu à ce résultat, il prend contact avec le boss voisin, il attire par son zèle, par son habileté et avant tout par sa discrétion, l'attention des anciens dans la carrière et désormais la voie lui est ouverte pour gravir les différents échelons. Le boss est ainsi devenu un élément indispensable du [152] parti, car tout est centralise en ses mains. C'est lui qui procure en très grande partie les moyens financiers. Mais comment s'y prend-il pour les obtenir? Partiellement par les cotisations des membres, mais avant tout par une taxe dont il frappe les traitements des fonctionnaires qui, grâce à lui et à son parti, ont trouvé une situation. De plus, il y a également les pots-de-vin et les pourboires. Celui qui a l'intention de violer impunément une des nombreuses lois des États doit auparavant obtenir la connivence des bosses et leur verser une certaine somme d'argent sous peine de se heurter aux pires inconvénients. Mais toutes ces diverses ressources ne suffisent point à constituer le capital nécessaire à l'entreprise politique du parti. Le boss est l'homme indispensable pour recueillir directement les fonds que les grands magnats de la finance versent à son organisation. Ceux-ci ne confieraient jamais une somme d'argent destinée à des fins électorales à un fonctionnaire rétribué du parti ni en général à aucune personne officiellement comptable du budget du parti ; mais le boss, à cause de sa prudence et de sa dis- 
crétion en matière d'argent, est évidemment l'homme clés milieux capitalistes qui financent les élections. Le boss typique est généralement un homme qui sait ce qu'il veut. Il ne recherche pas les honneurs; le professional (c'est ainsi qu'on l'appelle) est certes méprisé par la «bonne société ». Il ne recherche exclusivement que le pouvoir, soit comme source d'argent soit aussi pour lui-même. Contrairement au leader anglais il travaille dans l'obscurité. On ne l'entendra pas parler lui-même en publie; il suggère aux orateurs ce qu'il convient de dire mais lui-même se tiendra coi. En règle générale il n'accepte aucun poste officiel, sauf celui de sénateur. En effet, puisqu'en vertu de la constitution les sénateurs ont leur mot à dire dans le patronage des emplois, les bosses dirigeants siègent souvent en personne dans cette assemblée. La distribution des [153] postes se fait surtout d'après les services rendus au parti. Mais il arrive fréquemment que l'attribution se fasse également contre versement d'une somme d'argent et il existe même des tarifs précis pour pouvoir obtenir tel ou tel poste défini. Bref, il s'agit d'un système de vénalité des charges qu'avaient souvent pratiqué les monarchies du $\mathrm{XVII}^{\mathrm{e}}$ et du $\mathrm{XVIII}^{\mathrm{e}}$ siècles, y compris des États 'de l'Église.

Le boss n'a pas de « doctrine » politique ferme ; il ne connaît pas de principes. Une seule chose compte à ses yeux : comment faire pour ramasser le plus de voix possible ? Il arrive fréquemment qu'à soit un être sans grande éducation. Mais habituellement il mène une vie privée correcte et irréprochable. Ce n'est évidemment qu'en matière de morale politique qu'il s'adapte aux mœurs en vigueur dans ce secteur ; en cela il ne diffère guère d'un très grand nombre de nos capitalistes qui, en cette époque d'accapareurs, n'agissent pas autrement dans le domaine de la morale économique. Peu lui chaut que socialement on le traite de professional, de politicien professionnel. Du moment qu'il n'accède pas et ne veut pas accéder lui-même aux plus hauts postes de l'Union, sa modestie offre en même temps un certain nombre d'avantages : en effet, il n'est pas rare de voir que des intelligences étrangères au parti, des personnalités, soient présentées comme candidats aux élections si les bosses pensent qu'elles peuvent augmenter les chances électorales du parti. Situation bien différente de la nôtre où ce sont toujours les vieux notables du parti qu'on présente comme candidats. Pour cette raison, la structure de ce genre de partis, sans base doctrinale mais animés par des détenteurs du pouvoir méprisés par la 
société, a contribué à porter à la Présidence de l'Union des hommes de valeur qui n'auraient jamais "percé » chez nous. Assurément les bosses se dressent contre l'outsider qui pourrait menacer en cas d'élection leurs sources de [154] revenus et de pouvoir. Mais en raison même de la concurrence pour gagner la faveur des électeurs ils ont quelquefois été obligés de se résigner et d'accepter justement des candidats qui passaient pour des adversaires de la corruption.

Nous sommes donc dans ce cas en présence d'une entreprise politique ayant une forte structure capitaliste, organisée de façon rigide de haut en bas et soutenue par des clubs extrêmement puissants tels que Tammany Hall. Ces clubs, organisés à la manière d'un ordre, ne cherchent qu'à tirer profit de la domination politique, particulièrement de l'administration municipale - qui constitue également en Amérique le plus gros morceau des dépouilles. Cette organisation des partis n'était possible que parce que les États-Unis étaient un pays démocratique et parce qu'ils étaient «pays neuf ». Mais cette conjoncture privilégiée fait que de nos jours ce système est condamné à mourir lentement. L'Amérique ne peut plus être gouvernée uniquement par des dilettantes. Il y avait environ quinze ans, lorsqu'on demandait aux ouvriers américains pourquoi ils pouvaient se laisser gouverner par des hommes que, de leur propre aveu, ils méprisaient, on obtenait cette réponse : «Nous préférons être administrés par des fonctionnaires sur lesquels nous pouvons cracher plutôt que par cette caste de fonctionnaires qui, comme chez vous, crache sur nous. " C'était là le vieux point de vue de la " démocratie » américaine, mais déjà à cette époque les milieux socialistes de ce pays avaient une autre opinion. Cette situation n'est plus tolérable aujourd'hui. L'administration des dilettantes ne répond plus aux conditions nouvelles du pays et le Civil Service Reform crée déjà, en nombre toujours plus grand, des postes de fonctionnaires de carrière avec le bénéfice de la retraite. De ce fait les fonctionnaires formés dans les universités et qui seront tout aussi incorruptibles que les nôtres pourront occuper les postes. [155] Déjà 100000 emplois environ ne constituent plus le butin du tournoi électoral, mais donnent droit à une retraite en même temps qu'ils sont soumis à des exigences de qualification. Cette nouvel-le formule fera reculer lentement et progressivement le spoil system. En conséquence, la structure de la direction des partis se transformera sans doute également, sans que l'on puisse encore prévoir le sens. 
En Allemagne, -les conditions déterminantes de l'entreprise politique ont été jusqu'à présent les suivantes. Tout d'abord, impuissance du Parlement. Il en résulte qu'aucune personnalité ayant un tempérament de chef n'y entre jamais pour une longue durée. Supposons qu'un tel homme veuille y entrer - que peut-il y faire ? Lorsqu'une place devient vacante à la chancellerie il peut dire au directeur du personnel de qui dépend la nomination : j'ai sous la main, dans ma circonscription électorale, un homme très capable qui peut faire votre affaire ; prenezle. Et les choses se passent très souvent ainsi. Mais c'est à peu près tout ce qu'un parlementaire allemand peut obtenir pour satisfaire ses instincts de puissance - si jamais il en a.

À cela s'ajoute un second facteur qui conditionne le premier, à savoir l'importance énorme du fonctionnaire de carrière en Allemagne. Dans ce domaine nous fûmes sans doute les premiers au monde. Mais il résulta de cette situation que les fonctionnaires prétendirent occuper non seulement les postes de fonctionnaires, mais aussi ceux de ministres. N'avons-nous pas entendu dire l'an dernier au Landtag bavarois, lors du débat sur l'introduction du parlementarisme, que si jamais on attribuait les postes ministériels aux parlementaires les fonctionnaires capables quitteraient la carrière ! Enfin, il faut ajouter que chez nous l'administration de la fonction publique se dérobait systématiquement au contrôle des commissions parlementaires, à l'opposé de ce qui se passe [156] en Angleterre. De ce fait on mettait le Parlement dans l'impossibilité - sauf en quelques rares exceptions - de former en son sein des chefs politiques capables de diriger réellement une administration.

Le troisième facteur, totalement différent de ce qui se passe en Amérique, est qu'en Allemagne il existe clés partis qui ont une doctrine politique de sorte qu'ils peuvent affirmer, au moins avec une bona fides subjective, que leurs membres sont les représentants d'une « conception du monde ». Mais les deux partis les plus importants de ce type, le Centrum d'un côté et la social-démocratie de l'autre, sont malheureusement des partis qui pour le moment sont nés pour être minoritaires, et se veulent ainsi. En effet les milieux dirigeants du Centrum dans l'Empire allemand n'ont jamais caché qu'ils s'opposaient au parlementarisme parce qu'ils craignaient d'être le dindon de la farce et qu'ils auraient plus de difficultés qu'actuellement pour faire pression sur le gouvernement chaque fois qu'ils veulent faire nommer à une 
fonction publique un homme de leur bord. La social-démocratie est un parti minoritaire par principe et elle constitua de ce fait un obstacle à la parlementarisation parce qu'elle ne voulait pas se souiller au contact de l'ordre établi auquel elle reprochait d'être bourgeois. Le fait que ces deux partis s'excluaient du système parlementaire a été la cause principale pour laquelle il fut impossible de l'introduire en Allemagne.

Quel est, dans ces conditions, le sort des hommes politiques professionnels en Allemagne? Ils n'ont jamais eu ni pouvoir ni responsabilité ; ils ne pouvaient donc jouer qu'un rôle assez subalterne de notables. Ce n'est que depuis peu qu'ils sont animés par ces instincts d'avenir qui sont si caractéristiques dans les autres pays. Comme les notables faisaient de leur petit fromage le but de leur vie, il était impossible à un homme qui ne leur ressemblait pas de s'élever. Je pourrais citer [157] de nombreuses carrières politiques dans chaque parti, social-démocratie comprise évidemment, qui ne sont rien d'autre que des tragédies, parce que les individus en question possédaient des qualités de chef, mais n'ont pas été pour cette raison tolérés par les notables. Tous nos partis ont ainsi emboîté le pas à la coterie des notables. Bebel par exemple était encore un chef, par son tempérament et par son humeur, bien que son intelligence fût modeste. Le fait qu'il ait été un martyr, qu'il ne décevait jamais la confiance des masses (évidemment aux yeux des masses) eut pour conséquence qu'elles le suivirent sans autres façons et que jamais ne put surgir à l'intérieur de son parti, une opposition sérieuse capable de lui faire échec. Mais la situation prit fin avec sa mort et le règne clés fonctionnaires commença. On vit apparaître à la surface les fonctionnaires syndicaux, les secrétaires du parti, les journalistes : le parti passa ainsi sous la coupe clés instincts bureaucratiques. Ce furent certes des fonctionnaires très honorables, peut-être même extraordinairement honorables quand on les compare à ceux d'autres pays, principalement aux fonctionnaires syndicaux souvent corruptibles d'Amérique. Il n'en resta pas moins que les conséquences de la domination des fonctionnaires, que nous venons d'analyser, se firent jour également dans ce parti. 
Depuis 1880 environ, les partis bourgeois ne furent plus que des coteries de notables. Occasionnellement ils furent, il est vrai, obligés de faire appel, pour des fins de propagande, à des intelligences étrangères au parti, ce qui leur permettait de proclamer : «Untel ou Untel est avec nous! » Mais dans la mesure du possible on prenait toutes les garanties pour empêcher ces noms-là de se présenter aux élections. Ce n'est que dans le cas où ils refusaient de se prêter à la manœuvre [158] que l'on se résignait à poser leur candidature. Au Parlement régnait le même état d'esprit. Nos groupes parlementaires étaient des coteries et ils -le sont restés. Tous les discours prononcés en séance plénière du Reichstag sont soumis à la censure préalable des partis. On le constate à l'ennui mortel qu'ils font naître. N'a le droit de prendre la parole que le député préalablement désigné. On ne peut concevoir plus grand contraste avec les mœurs parlementaires anglaises, aussi bien - pour des raisons diamétralement opposées - qu'avec les mœurs françaises.

Un changement est peut-être présentement en cours après le violent bouleversement que l'on se plait à appeler révolution. Je dis peut-être, mais cela n'est pas du tout certain. Tout d'abord on préconise maintenant la constitution de partis nouveaux. Mais ces nouvelles formations ne sont au départ que des appareils d'amateurs. Ce sont en particulier les étudiants clés grandes écoles qui ont milité le plus souvent pour cela. Ils allaient trouver un homme en qui ils croyaient avoir découvert des qualités de chef pour lui tenir ce langage : nous vous mâcherons la besogne et vous n'aurez plus qu'à l'exécuter. Mais il y eut également des appareils politiques à caractère commercial. Il est arrivé que certaines personnes se soient présentées chez des individus en qui elles croyaient également avoir découvert des qualités de chef pour leur offrir de s'occuper du recrutement des partisans contre versement d'une somme déterminée pour toute nouvelle voix électorale. Si maintenant vous me demandiez de vous dire honnêtement lequel de ces deux procédés me parait le plus sûr du point de vue de la technique politique, je crois que je donnerais la préférence au dernier. Mais dans les deux cas nous n'avons eu affaire qu'à des bulles de savon qui s'élevèrent très rapidement dans l'air pour éclater aussitôt. On se contenta de remanier les appareils déjà existants qui recommencèrent à fonctionner [159] comme autrefois. En réalité les deux phénomènes que nous venons de signaler ne sont que des symptômes qui indiquent que des appareils nouveaux pourraient peut-être bien voir le jour, à condi- 
tion toutefois qu'il y ait des chefs. Cependant les particularités techniques du système de la représentation proportionnelle empêcheront ces nouvelles formations de prendre leur essor. La seule chose que l'on ait vue apparaître jusqu'à présent, c'est simplement une paire de dictateurs qui ameutèrent la rue et qui disparurent aussi rapidement. Cependant seuls les partisans de ces dictateurs étaient vraiment organisés et obéissaient à une stricte discipline : d'où la force de ces minorités qui sont cependant en perte de vitesse, pour le, moment.

Supposons que l'on puisse modifier la situation. Il faudrait alors, après tout ce que nous venons de dire, prendre conscience du fait important suivant : quand les partis sont dirigés et animés par des chefs plébiscitaires, il s'ensuit une "perte d'âme » [Entseelung] ou encore, plus clairement, une prolétarisation spirituelle chez ses partisans. Les partisans organisés dans un appareil de ce genre ne peuvent être utiles aux chefs que s'ils leur obéissent aveuglément, c'est-à-dire s'ils sont, comme en Amérique, embrigadés dans une machine que ne perturbe ni la vanité des notables ni la prétention à l'originalité personnelle. L'élection de Lincoln n'a été possible que parce que l'organisation de son parti avait ce caractère; le même phénomène s'est produit, comme nous l'avons vu, avec le caucus au profit de Gladstone. Voilà précisément le prix dont il faut payer l'installation de vrais chefs à la tète d'un parti. Nous n'avons que le choix : ou bien une démocratie admet à sa tête un vrai chef et par suite accepte l'existence d'une " machine », on bien elle renie les chefs et elle tombe alors sous la domination des «Politiciens de métier» sans vocation [Berufspolitiker ohne Beruf] qui ne possèdent [160] pas les qualités charismatiques profondes qui font les chefs. Dans ce dernier cas nous avons affaire à ce que l'opposition à l'intérieur d'un parti appelle le règne des «factions ». Pour l'instant nous ne voyons au sein des partis allemands que la domination des politiciens. La perpétuation de cet état de choses, du moins dans l'État, fédéral, sera favorisée d'abord par le fait qu'on va sans doute ressusciter le Conseil fédéral [Bundesrat]. La conséquence nécessaire en sera une limitation du pouvoir de l'Assemblée [Reichstag], et en même temps l'impossibilité d'y voir, apparaître des chefs. Cette situation trouvera un terrain encore plus favorable avec le système de la représentation proportionnelle tel qu'on le conçoit pour l'instant. Ce système est en effet la manifestation typique d'une démocratie sans chefs, non seulement parce qu'il facilite le maquignonnage 
dans la confection des listes au profit des notables, mais aussi parce qu'il donne désormais aux groupements d'intérêts la possibilité de contraindre les formations politiques à prendre sur les listes quelquesuns de leurs employés, de sorte que l'on finit par se trouver devant un Parlement apolitique dans lequel les vrais chefs n'ont plus leur place. Seul le Président du Reich, à condition qu'il soit élu par plébiscite et non par le Parlement, pourrait devenir la soupape de sûreté face à la carence des chefs. Il n'est possible de faire surgir des chefs et d'opérer leur sélection qu'à la condition de leur laisser la possibilité de faire la preuve de leurs capacités en les mettant d'abord à l'épreuve d'une gestion municipale, en leur laissant le droit de choisir eux-mêmes leur entourage, comme cela se passe aux États-Unis lorsqu'on voit apparaître sur la scène un maire plébiscitaire décidé à s'attaquer à la corruption. Tel est finalement le résultat qu'on pourrait espérer si 1'on organisait les partis en fonction d'élections de ce type. Mais l'hostilité proprement petite-bourgeoise à l'égard des chefs qui anime tous les [161] partis, y compris et surtout la social-démocratie, laisse dans le vague la nature de l'organisation future des partis, en même temps que les chances que nous venons d'indiquer.

C'est pourquoi il n'est absolument pas possible de prévoir aujourd'hui quelle sera extérieurement la tournure que prendra l'activité politique étendue comme " vocation », et cela d'autant moins que l'on ne voit pas comment on pourrait offrir à ceux qui sont doués pour la politique la chance d'être placés devant une tâche politique satisfaisante. Celui qui, en raison de sa situation de fortune, sera obligé de vivre « de » la politique ne peut pas échapper à l'alternative suivante : ou bien s'engager dans la voie du journalisme et de la bureaucratie des partis, ou bien essayer d'occuper un poste dans une association, qui se charge de la défense de certains intérêts, par exemple les syndicats, les chambres de commerce, les chambres d'agriculture, les chambres de métiers, les bourses du Travail, les offices de placement, etc., ou encore un poste idoine dans une municipalité. On ne peut dire rien de plus sur cet aspect extérieur dans la profession politique sinon que le fonctionnaire d'un parti politique partage avec le journaliste l'odium 
du « déclassé ». Ils se verront toujours traités, ne fût-ce que par sousentendus, l'un de « scribe à gages » et l'autre d'« orateur à gages ». Celui qui ne peut faire face en son for intérieur à ces injures et qui est incapable de se donner à lui-même la bonne réponse ferait mieux de ne pas entrer dans de pareilles carrières qui, outre quelques pénibles tentations, ne lui offriront dans tous les cas que des déceptions continuelles.

Quelles sont maintenant les joies intimes que la carrière politique peut donner à celui qui [162] l'embrasse et quelles conditions préalables faut-il lui supposer?

Eh bien! elle procure d'abord le sentiment de la puissance. La conscience d'exercer une influence sur les autres humains, le sentiment de participer au pouvoir et surtout la conscience d'être du nombre de ceux qui tiennent en main un nerf important de l'histoire en train de se faire, peuvent élever l'homme politique professionnel, même celui qui n'occupe qu'une position modeste, au-dessus de la banalité de la vie quotidienne. Mais il se pose à ce propos la question suivante : quelles sont les qualités qui lui permettent d'espérer d'être à la hauteur du pouvoir qu'il exerce (si petit soit-il), et par conséquent à la hauteur de la responsabilité que ce pouvoir lui impose ? Cette question nous conduit dans la sphère des problèmes éthiques. En effet, c'est à cet ordre d'idées qu'appartient la question : quel homme faut-il être pour avoir le droit d'introduire ses doigts dans les rayons de la roue de l'histoire?

On peut dire qu'il y a trois qualités déterminantes qui font l'homme politique : la passion - le sentiment de la responsabilité - le coup d'œil, Passion au sens d'« objet à réaliser », [Schlichkeit], c'est-à-dire dévouement passionné à une «cause ", au dieu ou au démon qui en est le maître. Cela n'a rien à voir avec cette conduite purement intérieure que mon regretté ami Georges Simmel avait l'habitude d'appeler une " excitation stérile ». Conduite particulière à une certaine sorte d'intellectuels, de préférence russes (pas tous, il est vrai), et qui fait actuellement fureur dans nos milieux d'intellectuels obnubilés par ce carnaval que l'on décoré du nom pompeux de « révolution ». Tout cela n'est que " romantisme de ce qui est intellectuellement intéressant», d'où le sentiment objectif de la [163] responsabilité est absent. Ce 
n'est qu'un sentiment qui tourne à vide. En effet la passion seule, si sincère soit-elle, ne suffit pas. Lorsqu'elle est au service d'une cause sans que nous fassions de la responsabilité correspondante l'étoile polaire qui oriente d'une façon déterminante notre activité, elle ne fait pas d'un homme un chef politique. Il faut enfin le coup d'œil qui est la qualité psychologique déterminante de l'homme politique. Cela veut dire qu'il doit posséder la faculté de laisser les faits agir sur lui dans le recueillement et le calme intérieur de l'âme et par conséquent savoir maintenir à distance les hommes et les choses. "L'absence de détachement »[Distanz] comme telle est un des péchés mortels de l'homme politique. Si jamais on inculquait à notre jeune génération d'intellectuels le mépris à l'égard du détachement indispensable, on la condamnerait à l'impuissance politique. Le problème suivant se pose alors : comment peut-on faire cohabiter dans le même individu la passion ardente et le froid coup d'œil ? On fait la politique avec la tête et non avec les autres parties du corps ou de l'âme. Et pourtant, si le dévouement à une cause politique est autre chose qu'un simple jeu frivole d'intellectuel, mais une activité menée avec sincérité, il ne peut avoir d'autre source que la passion et il devra se nourrir de passion. Mais ce pouvoir de dompter son âme avec énergie, qui caractérise l'homme politique passionné et qui le distingue du simple dilettante de la politique gonflé uniquement d'excitation stérile, n'a de sens qu'à la condition d'acquérir l'habitude du détachement - dans tous les sens du mot. Ce que l'on appelle la «force » d'une personnalité politique signifie en tout premier qu'elle possède cette qualité.

C'est un ennemi bien vulgaire, trop humain, que l'homme politique doit vaincre chaque jour et chaque heure : la très ordinaire vanité. Elle est l'ennemi mortel de tout dévouement à une cause [164] et de tout détachement, et dans ce cas du détachement de soi-même.

La vanité est un trait commun et personne n'en est peut-être entièrement exempt. Dans les milieux scientifiques et universitaires elle est même une sorte de maladie professionnelle. Mais chez le savant, si antipathique soit-elle lorsqu'elle se manifeste, elle est relativement inoffensive, en ce sens qu'en règle générale elle ne trouble pas l'activité scientifique. Il en va tout autrement chez l'homme politique. Le désir du pouvoir est pour lui un moyen inévitable. L'« instinct de puissance » - comme l'on dit couramment - est en fait une de ses qualités normales. Aussi le péché contre le Saint-Esprit de sa vocation 
consiste-t-il dans un désir de puissance [Machtstreben] sans objectif qui, au lieu de se mettre exclusivement au service d'une " cause ", n'est que prétexte à griserie personnelle. En effet, il n'existe tout compte fait que deux sortes de péchés mortels en politique : ne défendre aucune cause et n'avoir pas le sentiment de sa responsabilité deux choses qui sont souvent, quoique pas toujours, identiques. La vanité ou, en d'autres termes, le besoin de se mettre personnellement, de la façon la plus apparente possible, au premier plan, induit le plus fréquemment l'homme politique en tentation de commettre l'un ou l'autre de ces péchés ou même les deux à la fois. D'autant plus que le démagogue est obligé de compter avec « l'effet qu'il fait » - c'est pourquoi il court toujours le danger de jouer le rôle d'un histrion ou encore de prendre trop à la légère la responsabilité des conséquences de ses actes, tout occupé qu'il est par l'impression qu'il peut faire sur les autres. D'un côté, le refus de se mettre au service d'une cause le conduit à rechercher l'apparence et l'éclat du pouvoir au lieu du pouvoir réel ; de l'autre côté, l'absence du sens de la responsabilité le conduit à ne jouir que du pouvoir pour lui-même, sans [165] aucun but positif. En effet bien que, on plutôt parce que la puissance est le moyen inévitable de la politique et qu'en conséquence le désir du pouvoir est une de ses forces motrices, il ne peut y avoir de caricature plus ruineuse de la politique que celle du matamore qui joue, avec le pouvoir à la manière d'un parvenu, ou encore Narcisse vaniteux de son pouvoir, bref tout adorateur du pouvoir comme tel. Certes le simple politicien de la puissance [Machtpolitiker], à qui l'on porte aussi chez nous un culte plein de ferveur, peut faire grand effet, mais tout cela se perd dans le vide et l'absurde. Ceux qui critiquent la a politique de puissance $»$ ont entièrement raison sur ce point. Le soudain effondrement moral de certains représentants typiques de cette attitude nous a permis d'être les témoins de la faiblesse 'et de l'impuissance qui se dissimulent derrière certains gestes pleins d'arrogance, mais parfaitement vides. Une pareille politique n'est jamais que le produit d'un esprit blasé, souverainement superficiel et médiocre, fermé à toute signification de l'activité humaine ; rien n'est d'ailleurs plus éloigné de la conscience du tragique qu'on trouve dans toute action et tout particulièrement dans l'action politique que cette mentalité.

Il est une chose incontestable, et c'est même un fait fondamental de l'histoire, mais auquel nous ne rendrons pas justice aujourd'hui : le ré- 
sultat final de l'activité politique répond rarement à l'intention primitive de Facteur. On peut même affirmer qu'en règle générale il n'y répond jamais et que très souvent le rapport entre le résultat final et l'intention originelle est tout simplement paradoxal. Mais cette constatation ne peut servir de prétexte pour s'abstenir (le se mettre au service d'une cause, car l'action perdrait alors toute consistance interne. Quant à la nature même de la cause au nom de laquelle l'homme politique cherche et utilise le pouvoir, nous ne pouvons rien en dire : elle dépend des [166] convictions personnelles de chacun. L'homme politique peut chercher à servir des fins nationales ou humanitaires, des fins sociales, éthiques ou culturelles, profanes ou religieuses. Il peut également être soutenu par une solide croyance au "progrès » - dans les différents sens de ce terme - comme il peut récuser absolument cette croyance ; il peut prétendre vouloir servir une " idée » ou refuser par principe la valeur des idées pour ne servir que les fins matérielles de la vie quotidienne. Dans tous les cas cependant, une croyance ou une foi est nécessaire, sinon - et personne ne peut le nier - le succès politique apparemment le plus solide rejoindra dans la malédiction l'inanité de la créature.

Ce que nous venons d'exposer nous met déjà la voie de la discussion du dernier problème qui nous occupera ce soir, celui de l'ethos de la politique, en tant que « cause » à défendre. Quelle est, indépendamment des buts qui lui sont propres, la mission que la politique peut remplir dans l'économie globale de la conduite de la vie ? Quel est pour ainsi dire le lien éthique où elle réside ? Sur ce point, les conceptions du monde les Plus opposées se heurtent les unes aux autres et finalement il faut choisir entre elles. Attaquons donc résolument ce problème que l'on a remis récemment sur le tapis, mais, à mon avis, en le prenant du mauvais côté.

Débarrassons-nous tout d'abord d'une contrefaçon vulgaire. L'éthique peut parfois jouer un rôle extrêmement fâcheux. Quelques exemples. Il n'est pas rare qu'un homme qui abandonne sa femme pour une autre éprouve le besoin de se justifier devant sa conscience 
en invoquant comme prétexte qu'elle n'était pas digne de son amour, qu'elle l'avait déçu, ou d'autres raisons de ce genre qui ne manquent pas. Il s'agit là de la [167] part de l'homme d'un manque de courtoisie qui, en plus de la simple constatation qu'il n'aime plus sa femme alors qu'elle est en l'occurrence la victime -, cherche encore à se fabriquer un alibi pour "justifier» son attitude profondément discourtoise : il s'arroge ainsi un droit qui laisse en fin de compte tous les torts à sa femme, en plus de l'infidélité dont il l'accable. Le vainqueur de cette rivalité sexuelle [erotisch] procède exactement de la même façon: il estime que son adversaire malheureux doit être le moins digne, puisqu'il est évincé. Il n'y a-là évidemment aucune différence avec le vainqueur qui, après sa victoire sur le champ de bataille, proclame avec la vile manie de celui qui a toujours raison [Rechthaberei] : « J'ai vaincu parce que j'avais raison. » Il en est de même de l'homme qui, à la vue des atrocités de la guerre, s'effondre moralement et qui au lieu de dire simplement : "C'est vraiment trop, je ne puis plus le supporter» - éprouve le besoin, pour se justifier devant sa propre conscience, de substituer à ce sentiment de lassitude devant la guerre un tout autre sentiment en prétextant : "je ne pouvais plus supporter tout cela parce qu'on m'obligeait à combattre pour une cause moralement injuste. » On peut dire la même chose de celui qui est vaincu ; au lieu de se complaire dans l'attitude des vieilles femmes et chercher à découvrir le " coupable » après la défaite - puisque c'est toujours la structure même de la société qui engendre les conflits - il ferait mieux d'adopter une attitude virile et digne en disant à l'ennemi : "Nous avons perdu la guerre et vous l'avez gagnée. Oublions le passé et discutons maintenant des conséquences qu'il faut tirer de la situation nouvelle en considérant les intérêts matériels qui étaient en jeu et question essentielle - en considérant la responsabilité devant l'avenir qui pèse en premier lien sur le vainqueur. » Tout le reste dénote simplement une absence de dignité et se paiera un jour ou l'autre. Une nation pardonne toujours [168] les préjudices matériels qu'on lui fait subir, mais non une atteinte portée à son honneur, surtout lorsqu'on emploie la manière d'un prédicateur qui veut avoir raison à tout prix. Tout nouveau document porté à la connaissance publique des dizaines d'années après ne peut avoir d'autre résultat que de réveiller des clameurs indignes, la colère et la haine, alors qu'il vaudrait mieux enterrer la guerre, moralement au moins, lorsqu'elle est terminée. Mais une telle attitude n'est possible que si l'on a le sens des réalités, le sens 
chevaleresque et avant tout le sens de la dignité. Elle se défend donc d'afficher une « éthique » qui, en vérité, témoigne toujours d'une absence de dignité des deux côtés. Ce dernier genre d'éthique ne se préoccupe jamais que de la culpabilité dans le passé, question stérile du point de vue politique parce qu'insoluble, au lien de s'intéresser à l'affaire propre de l'homme politique, à savoir l'avenir et la responsabilité devant l'avenir. S'il existe des crimes politiques, cette façon de procéder en est un. Par ailleurs, une telle attitude a l'inconvénient supplémentaire de nous cacher à quel point tout le problème est inévitablement faussé par des intérêts matériels : intérêts du vainqueur à tirer le plus grand profit possible de sa victoire - qu'il s'agisse d'intérêts matériels ou moraux -, espoirs du vaincu de marchander l'aveu de sa culpabilité contre certains avantages. S'il existe au monde quelque chose d'« abject» c'est bien cela. Voilà ce qu'il en résulte lorsque l'on veut utiliser l'éthique pour avoir toujours raison.

Mais comment se pose alors le problème des relations véritables entre éthique et politique? N'existe-t-il absolument aucun rapport entre ces deux sphères, comme on l'a dit quelquefois ? On bien seraitil plus juste au contraire de dire que la même éthique est valable aussi bien pour l'action politique que pour n'importe quelle autre espèce d'action ? On a cru parfois qu'il existait [169] une opposition absolue entre les deux thèses : ou bien l'une est exacte, ou bien l'autre. Mais on peut se demander s'il existe au monde une éthique capable d'imposer des obligations identiques, quant à son contenu, à la fois aux relations sexuelles, commerciales, privées et publiques, aux relations d'un homme avec son épouse, sa marchande de légumes, son fils, son concurrent, son ami et son ennemi. Peut-on vraiment croire que les exigences de l'éthique puissent rester indifférentes au fait que toute politique utilise comme moyen spécifique la force, derrière laquelle se profile la violence ? Ne constatons-nous pas que, parce que les idéologues du bolchevisme et du spartakisme ont précisément en recours à la violence, ils aboutissent exactement aux mêmes résultats que n'importe quel autre dictateur militaire ? En quoi la domination des "Conseils d'ouvriers et de soldats " se distingue-t-elle de celle de n'importe quel autre détenteur du pouvoir Cie l'ancien régime impérial, sinon par le simple fait que d'autres chefs détiennent le pouvoir, qui sont de simples dilettantes ? En quoi la polémique de la plupart des défenseurs de la prétendue nouvelle éthique, même lorsqu'ils cri- 
tiquent celle de leurs adversaires, est-elle différente de celle de n'importe quel autre démagogue ? Par la noble intention dira-t-on. Bien. Mais ce qui est en question ici, c'est le moyen, car les adversaires qu'ils combattent revendiquent exactement de la même façon, avec la même et entière sincérité subjective, la noblesse de leurs intentions ultimes. «Celui qui tire l'épée périra par l'épée » et la lutte est partout la lutte. Et alors ? - L'éthique du Sermon sur la Montagne? Mais le Sermon sur la Montagne - j'entends par-là l'éthique absolue de l'Évangile - est une chose beaucoup plus sérieuse que ne le croient ceux qui de nos jours citent volontiers ses commandements. On ne plaisante pas avec elle. Ce que l'on a dit à propos de la causalité en science s'applique également à [170] l'éthique : elle n'est pas un fiacre que l'on petit faire arrêter à son gré pour y monter ou en descendre suivant le cas. À moins de n'y voir qu'un recueil de trivialités, l'éthique de l'Évangile est une morale du « tout ou rien ». La parabole du jeune riche nous dit par exemple : a Et il s'en alla le cœur triste, car il possédait beaucoup de biens. » Le commandement de l'Évangile est inconditionnel et univoque : donne tout ce que tu possèdes - absolument tout, sans réserve. L'homme politique dira que ce commandement n'est qu'une exigence sociale irréalisable et absurde aussi longtemps qu'il ne s'applique pas à tout le monde. En conséquence l'homme politique proposera la suppression de la propriété par taxation, imposition, confiscation, - bref la contrainte et la réglementation dirigée contre tout le monde. Mais le commandement éthique ne se soucie absolument pas de cela, c'est là son propre. Il dit encore : «Présente l'autre joue!» Immédiatement, sans demander à l'autre pourquoi il croit devoir te frapper. Éthique sans dignité, dira-t-on. Oui - sauf pour le saint. C'est bien cela : il faut être un saint en tout, ou du moins vouloir l'être, et vivre comme jésus, les Apôtres, saint François d'Assise et ses pareils, car alors elle a un sens et exprime une dignité. Dans le cas contraire elle n'en a pas. Par conséquent, si l'éthique a-cosmique de l'amour nous dit: "Ne résiste pas au mal par la force », l'homme politique au contraire dira : « Tu dois t'opposer au mal par la force, sinon tu es responsable de son triomphe. » Celui qui veut agir selon l'éthique de l'Évangile doit renoncer à faire grève - car la grève est une contrainte - et il ne lui reste d'autre solution que de s'affilier à un syndicat jaune. Et surtout qu'il s'abstienne de parler de " révolution »! En effet le but de cette éthique ne consiste tout de même pas à nous enseigner que la guerre civile serait la seule guerre légitime. Le pacifiste qui agit selon les préceptes 
de l'Évangile déposera les armes ou [171] les jettera au loin par respect pour le devoir éthique, ainsi qu'on l'a recommandé en Allemagne, afin de mettre fin à la guerre et en même temps à toute guerre. L'homme politique au contraire dira : le seul moyen sûr qui aurait permis de discréditer la guerre pour tout le futur prévisible aurait été une paix immédiate fondée sur le statu quo. En effet, les peuples se seraient alors demandé : à quoi nous a servi cette guerre ? L'on aurait mis ainsi en évidence son absurdité - solution qu'il n'est plus possible d'adopter maintenant. En effet la guerre aura été politiquement profitable aux vainqueurs, du moins à une partie d'entre eux. La responsabilité de cette situation incombe à l'attitude qui nous a privés de toute possibilité de cette résistance. Mais bientôt - lorsque la période de lassitude sera dépassée - ce ne sera plus la guerre qui sera discréditée, mais la paix : conséquence de l'éthique absolue.

Il y a enfin le devoir de vérité. Il est, lui aussi, inconditionnel du point de vue de l'éthique absolue. On en a tiré la conclusion qu'il fallait publier tous les documents, principalement ceux qui accablent son propre pays, pour mettre en évidence, sur -la foi de ces témoignages à sens unique, l'aveu d'une culpabilité unilatérale, inconditionnelle et sans égard aux conséquences. L'homme politique, trouvera que cette façon de faire, à la juger sur son résultat, loin de faire la lumière sur la vérité, l'obscurcira à coup sûr par les abus et le déchaînement des passions; il sait que seul l'établissement des faits élaborés méthodiquement, sans aucune exclusive, par des personnes impartiales pourrait porter des fruits et que toute autre méthode aura pour la nation usant de ces procédés des conséquences qui demanderont peut-être des années avant d'être redressées. À vrai dire, s'il existe un problème dont l'éthique absolue ne s'occupe pas, c'est bien celui qui concerne les conséquences !

[172]

Nous en arrivons ainsi au problème décisif. Il est indispensable que nous nous rendions clairement compte du fait suivant : toute activité orientée selon l'éthique peut être subordonnée à deux maximes totalement différentes et irréductiblement opposées. Elle peut s'orienter selon l'éthique de la responsabilité [verantwortungsethisch] ou selon l'éthique de la conviction [gesinnungsethisch]. Cela ne veut pas dire que l'éthique de conviction est identique à l'absence de responsabilité et l'éthique de responsabilité à l'absence de conviction. Il n'en est évi- 
demment pas question. Toutefois il y a une opposition abyssale entre l'attitude de celui qui agit selon les maximes de l'éthique de conviction - dans un langage religieux nous dirions : «Le chrétien fait son devoir et en ce qui concerne le résultat de l'action il s'en remet à Dieu »-, et l'attitude de celui qui agit selon l'éthique de responsabilité qui dit : " Nous devons répondre des conséquences prévisibles de nos actes. " Vous perdrez votre temps à exposer, de la façon la plus persuasive possible, à un syndicaliste convaincu de la vérité de l'éthique de conviction que son action n'aura d'autre effet que celui d'accroître les chances de la réaction, de retarder l'ascension de sa classe et de l'asservir davantage, il ne vous croira pas. Lorsque les conséquences d'un acte fait par pure conviction sont fâcheuses, le partisan de cette éthique n'attribuera pas la responsabilité à l'agent, mais au monde, à la sottise des hommes ou encore à la volonté de Dieu qui a créé les hommes ainsi. Au contraire le partisan de l'éthique de responsabilité comptera justement avec les défaillances communes de l'homme (car, comme le disait fort justement Fichte, on n'a pas le droit de présupposer la bonté et la perfection de l'homme) et il estimera ne pas pouvoir se décharger sur les autres des conséquences de sa propre action pour autant qu'il aura pu les prévoir. Il dira donc : «Ces conséquences sont imputables à ma propre action. » [173] Le partisan de l'éthique de conviction ne se sentira "responsable » que de la nécessité de veiller sur la flamme de la pure doctrine afin qu'elle ne s'éteigne pas, par exemple sur la flamme qui anime la protestation contre l'injustice sociale. Ses actes qui ne peuvent et ne doivent avoir qu'une valeur exemplaire mais qui, considérés du point de vue du but éventuel, sont totalement irrationnels, ne peuvent avoir que cette seule fin : ranimer perpétuellement la flamme de sa conviction.

Mais cette analyse n'épuise pas encore le sujet. Il n'existe aucune éthique au monde qui puisse négliger ceci : pour atteindre des fins «bonnes », nous sommes la plupart du temps obligés de compter avec, d'une part clés moyens moralement malhonnêtes ou pour le moins dangereux, et d'autre part la possibilité ou encore l'éventualité de conséquences fâcheuses. Aucune éthique au monde ne peut nous dire non plus à quel moment et dans quel-le mesure une fin moralement bonne justifie les moyens et les conséquences moralement dangereuses, 
Le moyen décisif en politique est la violence. On peut mesurer jusqu'où peut aller, du point de vue de l'éthique, la tension entre moyens et fin, lorsque l'on considéré l'attitude bien connue des socialistes révolutionnaires de la tendance Zimmerwald. Déjà pendant la guerre ils s'étaient déclarés en faveur d'un principe que l'on peut exprimer de façon frappante en ces termes : «En présence du choix : quelques années encore de guerre suivie d'une révolution, ou bien la paix immédiate non suivie d'une révolution - nous choisissons la solution : encore quelques années de guerre ! » À la question suivante : que peut apporter cette révolution ? - tout socialiste raisonnant scientifiquement d'après les principes de sa doctrine ne peut que donner cette réponse : il ne peut être question pour le moment d'un passage à une économie que l'on pourrait appeler socialiste dans [174] le sens propre du terme et une économie du type bourgeois verra de nouveau le jour après l'élimination des vestiges de la féodalité et des éléments dynastiques. C'est donc pour ce modeste résultat qu'on accepterait «encore quelques années de guerre »! On voudrait croire qu'il conviendrait, même si l'on est un socialiste convaincu, de renoncer à une fin qui met en œuvre de tels moyens. Le problème ne se pose pas autrement dans le cas du bolchevisme et du spartakisme, et en général dans le cas de n'importe quelle espèce de socialisme révolutionnaire, car il est parfaitement ridicule de la part des révolutionnaires de condamner au nom de la morale la «politique de force » des hommes de l'ancien régime alors qu'en fin de compte ils utilisent exactement le même moyen - si justifiée que soit par ailleurs leur position lorsqu'ils refusent les buts de leurs adversaires.

Il semble donc que c'est bien le problème de la justification des moyens par la fin qui voue en général à l'échec l'éthique de conviction. En fait, il ne lui reste logiquement d'autre possibilité que celle de repousser toute action qui fait appel à des moyens moralement dangereux. Je dis bien : logiquement. Car dans le monde des réalités nous constatons sans cesse par expérience que le partisan de l'éthique de conviction fait brusquement volte-face pour devenir un prophète millénariste et que les mêmes individus qui, quelques instants auparavant, avaient prêché la doctrine de l'« amour opposé à la force ", font justement appel quelques minutes plus tard à cette même force - à l'ultime force qui aboutira à l'anéantissement final de toute violence - à la manière de nos chefs militaires qui proclamaient lors de chaque nou- 
velle offensive : c'est la dernière, celle qui nous conduit à la victoire et qui nous apportera la paix. Le partisan de l'éthique de conviction ne peut supporter l'irrationalité éthique du monde. Il est un " rationaliste » cosmo-éthique. Ceux d'entre [175] vous qui connaissent Dostoïevski pourront évoquer ici la scène du Grand. Inquisiteur au cours de laquelle ce problème est exposé de façon pertinente. Il n'est pas possible de concilier l'éthique de conviction et l'éthique de responsabilité pas plus qu'il n'est possible de décréter au nom de la morale quelle est la fin qui justifiera tel moyen, si jamais on fait la moindre concession au principe.

Mon collègue P. W. Foerster, que personnellement j'estime beaucoup pour la sincérité incontestable de ses convictions mais à qui je refuse absolument la qualité d'homme politique, croit pouvoir tourner la difficulté dans un de ses ouvrages en préconisant cette simple thèse : le bien ne peut engendrer que le bien et le mal ne peut engendrer que le mal. S'il en était ainsi il n'y aurait plus de problème. Il est vraiment étonnant qu'une pareille thèse ait encore pu voir le jour deux mille cinq cents ans après les Upanishads. Ce n'est pas seulement tout le cours de l'histoire mondiale qui nous dit le contraire, mais également tout examen impartial de l'expérience quotidienne. Le développement de toutes les religions du monde est fondé sur la vérité de l'opinion inverse. Le très antique problème de la Théodicée se posait déjà la question : comment se fait-il qu'une puissance qu'on nous présente à la fois comme omnipotente et bonne ait pu créer un monde aussi irrationnel de souffrances non méritées, d'injustices non punies et de stupidité incorrigible ? Ou bien cette puissance est omnipotente et bonne ou bien elle ne l'est pas, on enfin des principes totalement différents de compensation et de sanction régissent la vie, principes qu'il n'est possible d'interpréter que par les voies de la métaphysique à moins qu'ils n'échappent complètement à notre pouvoir de compréhension. Ce problème de l'expérience de l'irrationalité du monde a été la force motrice du développement de toutes les religions. La doctrine hindoue du karman, celle du dualisme [176] persan, la doctrine du péché originel, de la prédestination et du Deus absconditus sont toutes nées de cette expérience. Les premiers chrétiens savaient très bien également que le monde était régi par des démons et que l'individu qui se compromettait avec la politique, c'est-à-dire avec les moyens de la puissance et de la violence, concluait un pacte avec des puissances diaboliques ; il 
n'est pas vrai, savaient-ils aussi, qu'au cours de leurs actions le 'bien engendre uniquement le bien et le mal uniquement le mal - l'on constate bien plutôt et très souvent le phénomène inverse. Qui ne voit pas cela est en vérité, politiquement, un enfant.

L'éthique religieuse s'est accommodée de diverses façons de cette situation fondamentale qui fait que nous sommes placés dans des régimes de vie différents, subordonnés à leur tour à des lois également différentes. Le polythéisme hellénique offrait des sacrifices à Aphrodite aussi bien qu'à Héra, à Apollon aussi bien qu'à Dionysos, et il savait que ces dieux se combattent fréquemment. Le système de vie hindou faisait de chacune des professions l'objet d'une loi éthique particulière, d'un dharma, et établissait pour toujours entre elles une séparation par castes et les intégrait ensuite dans une hiérarchie immuable. L'individu né dans une caste n'avait plus de possibilité de la déserter, sauf par une réincarnation dans la vie future. Par conséquent chaque profession se trouvait à une distance différente, plus ou moins importante, du salut suprême. On a donc établi le dharma de chacune des castes, depuis les ascètes et les brahmanes jusqu'aux fripons et aux catins, à l'intérieur d'une hiérarchie établie selon les lois immanentes, propres à chaque profession. La guerre et la politique y ont naturellement trouvé chacune sa place. Que la guerre soit partie intégrante de la vie, vous le verrez en lisant dans la Bhagavad Gitâ la conversation entre Krishna et Arjuna. "Fais ce qui est nécessaire », ce qui veut [177] dire : accomplis le devoir que t'impose le dharma de la caste des guerriers et les prescriptions qui la régissent, bref, accomplis l'« œuvre » objectivement nécessaire qui correspond au but de ta caste, à savoir, faire la guerre. Selon cette croyance, accomplir sa tâche de guerrier était loin de constituer un danger pour le salut de son âme, c'en était au contraire le soutien. De tout temps le guerrier hindou était tout aussi certain de rejoindre, après une mort héroïque, le ciel d'Indra que le guerrier germanique d'être accueilli dans le Walhalla ; il aurait sans doute dédaigné tout autant le nirvâna que le germanique le paradis chrétien avec ses chœurs d'anges. Cette spécialisation de l'éthique permit à la morale hindoue de faire de l'art royal de la politique une activité parfaitement conséquente, soumise à ses seules lois, toujours plus consciente d'elle-même. La littérature hindoue nous offre même un exposé classique du « machiavélisme » radical, au sens populaire du machiavélisme ; il suffit de lire l'Arthaçâstra de Kautilya 
qui fut écrit bien avant l'ère chrétienne, probablement sous Chandragupta. Comparé à ce document le Prince de Machiavel est un livre inoffensif. On sait que dans l'éthique catholique, dont le professeur Foerster est si proche par ailleurs, les consilia evangelica constituent une morale spéciale, réservée à ceux qui possèdent le privilège du charisme de la sainteté. On y trouve, à côté du moine, à qui il est interdit de verser du sang ou de rechercher un profit, les figures du chevalier et du bourgeois pieux qui avaient le droit, le premier de verser le sang et le second de s'enrichir. Sans doute, la différenciation de l'éthique et son intégration dans un système du salut y sont moins conséquentes qu'aux Indes; néanmoins, en vertu des présuppositions de la foi chrétienne, il pouvait et même il devait en être ainsi. La doctrine de la corruption du monde par le péché originel permettait d'intégrer avec une relative facilité la violence dans [178] l'éthique, en tant que moyen, pour combattre le péché et les hérésies qui constituent précisément des dangers pour l'âme. - Cependant les exigences a-cosmiques du Sermon sur la Montagne, sous la forme d'une pure éthique de conviction, ainsi que le droit naturel chrétien compris comme une exigence absolue fondée sur cette doctrine, ont conservé leur puissance révolutionnaire et ils sont chaque fois revenus à la surface avec toute leur fureur dans presque toutes les périodes de bouleversements sociaux. Ils ont notamment donné naissance à des sectes qui professent un pacifisme radical; l'une d'elles avait même essayé de construire en Pennsylvanie un État qui se refusait à utiliser la force dans ses relations extérieures - expérience qui fut d'ailleurs tragique dans son déroulement dans la mesure où, lors du déclenchement de la guerre d'Indépendance, les Quakers ne purent intervenir l'arme à la main dans un conflit dont l'objectif était pourtant la défense d'idéaux identiques aux leurs. Par contre le protestantisme commun légitime en général l'État et de ce fait le recours à la violence comme une institution divine, et il justifie tout particulièrement l'État autoritaire légitime. Luther a enlevé à l'individu la responsabilité éthique de là guerre pour l'attribuer à l'autorité politique, de sorte que l'obéissance aux ordres des pouvoirs Politiques ne peut être coupable, hormis les questions de foi. Le calvinisme reconnaissait lui aussi, en principe, la force comme moyen pour défendre la foi et par conséquent il légitimait les guerres de religion. On sait que depuis toujours celles-ci étaient un élément vital de l'Islam. On voit donc maintenant que ce n'est nullement l'incroyance moderne, issue du culte de la Renaissance pour les 
héros, qui a soulevé le problème de l'éthique politique. Toutes les religions ont débattu cette question avec plus on moins de succès, et notre exposé a suffisamment montré qu'il ne pouvait en être autrement. L'originalité propre aux problèmes [179] éthiques en politique réside donc dans le moyen spécifique de la violence légitime comme telle, dont disposent les groupements humains.

Quel que soit le but de son action, tout homme qui pactise avec ce moyen - et tout homme politique le fait nécessairement - s'expose aux conséquences qui en résultent. Cela est tout particulièrement vrai d'un individu qui combat pour ses convictions, qu'il s'agisse du militant religieux ou du militant révolutionnaire. Prenons hardiment l'époque contemporaine comme exemple. Quiconque veut instaurer par la force la justice sociale sur terre a besoin de partisans, c'est-à-dire d'un appareil humain. Or ce appareil ne marche que si on lui fait entrevoir les récompenses psychologiques ou matérielles indispensables, qu'elles soient célestes ou terrestres. Tout d'abord les récompenses psychologiques : dans les conditions modernes de la lutte de classes, ce sont la satisfaction de ses haines, de ses vengeances, de son ressentiment surtout et de son penchant pseudo-éthique à avoir raison à tout prix : par conséquent assouvir son besoin de diffamer l'adversaire et de l'accuser d'hérésie. ensuite les récompenses matérielles : aventure, victoire, butin, pouvoir et prébendes. Le succès du chef dépend entièrement du fonctionnement de son appareil. C'est pourquoi il dépend également des motifs qui animent les partisans et non de ceux qui l'animent lui personnellement. Son avenir dépend donc de la possibilité de procurer, de façon durable, toutes ces récompenses aux partisans dont il ne peut se passer, qu'il s'agisse de la garde rouge, des mouchards ou des agitateurs. Il n'est donc pas entièrement maître des résultats de son activité, il doit se plier aussi aux exigences de ses partisans, exigences que la morale peut trouver basses. Il les tient en bride aussi longtemps qu'une foi sincère en sa personne et en sa cause anime une fraction au moins de ses partisans, car on n'a jamais vu au monde que des sentiments identiques inspirent ne fût-ce [180] que la majorité d'un groupement humain. Tout d'abord ces convictions, même là où elles sont subjectivement les plus sincères, ne servent en réalité la plupart du temps qu'à «justifier » moralement les désirs de vengeance, de pouvoir, de butin et de prébendes. Sur ce point nous ne nous laisserons pas conter d'histoires, car l'interprétation matérialiste de l'histoire n'est 
pas non plus un fiacre dans lequel on peut monter à son gré et qui s'arrêterait devant les promoteurs de la révolution! Mais il ne faut surtout pas oublier qu'à la révolution pleine d'enthousiasme succédera toujours la routine quotidienne d'une tradition et qu'à ce moment-là le héros de la foi abdiquera et surtout la foi elle-même se résignera ou encore elle deviendra - et ce sera là son plus cruel destin - un élément de la phraséologie conventionnelle des cuistres et des techniciens de la politique. Cette évolution est tout particulièrement rapide dans les luttes idéologiques, tout simplement parce que ce genre de luttes est en général dirigé ou inspiré par des chefs authentiques, les prophètes de la révolution. Dans ce cas en effet, comme en général dans toute activité qui réclame un appareil à la dévotion du chef, l'appauvrissement et la mécanisation ou encore la prolétarisation spirituelle au profit de la «discipline » constituent une des conditions du succès. C'est pourquoi les partisans victorieux d'un chef qui combat pour ses convictions dégénèrent d'ordinaire très rapidement en une masse de vulgaires prébendiers.

Celui qui, en général, veut faire de la politique et surtout celui qui veut eu faire sa vocation doit prendre conscience de ces paradoxes éthiques et de responsabilité à l'égard de ce qu'il peut lui-même devenir sous leur pression. Je le répète, il se compromet avec des puissances diaboliques qui sont aux aguets dans toute violence. Les grands virtuoses de l'amour et de la bonté a-cosmiques de [181] l'homme, qu'ils nous viennent de Nazareth, d'Assise on des châteaux royaux des Indes, n'ont pas travaillé avec le moyen politique de la violence. Leur royaume n'était pas « de ce monde » et pourtant ils ont en et ils continuent à y exercer une influence. Les figures de Platon Karatajev et des saints de Dostoïevski sont certainement les reconstitutions les plus fidèles de ce genre de virtuoses. Celui qui veut le salut de son âme ou sauver celle des autres doit donc éviter les chemins de la politique qui, par vocation, cherche à accomplir d'autres tâches très différentes, dont on ne peut venir à bout que par la violence. Le génie ou le démon de la politique vit dans un état de tension extrême avec le Dieu de l'amour et aussi avec le Dieu des chrétiens tel qu'il se manifeste 
dans les institutions de l'Église. Cette tension peut en tout temps éclater en un conflit insoluble. Cela, les hommes le savaient même à l'époque de la domination de l'Église. Sans arrêt l'interdit frappait la ville de Florence - et en ce temps une telle pression pesait beaucoup plus lourdement sur les hommes et menaçait davantage le salut de leur âme que l'« approbation froide » (comme dit Fichte) du jugement moral kantien - mais les citoyens de la cité continuaient à faire la guerre aux États de l'Église. Dans une beau passage de ses Histoires florentines, si mes souvenirs sont exacts, Machiavel fait allusion à cette situation et met dans la bouche d'un héros de cette ville les paroles suivantes, pour rendre hommage à ses concitoyens : "Ils ont préféré la grandeur de leur cité au salut de leur âme. »

Si au lieu de cité natale ou de «patrie », mots qui n'ont plus de nos jours une signification univoque pour tout le monde, vous dites l'« avenir du socialisme» ou encore « la paix internationale », vous emploierez les termes qui correspondent à la façon moderne de poser le problème. En effet, tous ces buts qu'il n'est pas possible d'atteindre autrement que par l'activité politique, laquelle fait [182] nécessairement appel à des moyens violents et emprunte les voies de l'éthique de la responsabilité, mettent en péril le «salut de l'âme ». Et si l'on cherche à atteindre ces objectifs au cours d'un combat idéologique guidé par une éthique de conviction, il peut en résulter de grands dommages et un discrédit dont les répercussions se feront sentir pendant plusieurs générations, parce qu'il y manque la responsabilité des conséquences. Dans ce cas en effet, l'agent n'a plus conscience des puissances diaboliques qui entrent en jeu. Or celles-ci sont inexorables et si l'individu ne les voit pas il sera entraîné dans un certain nombre de conséquences auxquelles il sera livré sans merci ; leurs répercussions se feront sentir au cours de son action mais aussi au fond de son âme. « Le diable est vieux. » Et lorsque le poète ajoute " devenez vieux, vous aussi, pour pouvoir le comprendre », il ne pensait certainement pas au nombre des années ou à l'âge. Personnellement je n'ai jamais admis qu'au cours d'une discussion on cherchât à prendre l'avantage en exhibant son acte de naissance. Le simple fait qu'un de mes interlocuteurs a vingt ans alors que je dépasse la cinquantaine ne peut en fin de compte m'autoriser à penser que cela seul constituerait un exploit devant lequel je devrais m'incliner avec respect. Ce n'est pas l'âge qui importe, mais d'abord la souveraine compétence du re- 
gard qui sait voir les réalités de la vie sans fard et ensuite la force d'âme qui est capable de les supporter et de se sauver avec elles.

Il est vrai la politique se fait avec la tête. Mais il est tout aussi vrai qu'on ne la fait pas uniquement avec la tête. Sur ce point les partisans de l'éthique de conviction ont parfaitement raison. On ne peut prescrire à personne d'agit selon l'éthique de conviction ou selon l'éthique de responsabilité, pas plus qu'on ne peut lui indiquer à quel moment il doit suivre l'une et à quel moment l'autre, On ne peut dire qu'une seule chose. Quand aujourd'hui, en un temps d'excitations qui, selon [183] votre opinion, ne sont pas stériles - sachez cependant que l'excitation n'est pas toujours ni même foncièrement une passion authentique - on voit surgir subitement de partout des hommes politiques animés par l'esprit de l'éthique de conviction et qui proclament : «Ce n'est pas moi, mais le monde qui est stupide et vulgaire ; la responsabilité des conséquences n'incombe pas à moi, mais aux autres au service desquels je travaille ; cependant, attendez un peu, je saurai bien extirper cette stupidité et cette vulgarité », - alors je vous le dis très franchement, je commence d'abord par me renseigner sur l'équilibre intérieur [inneren Schwergewichts] de ces partisans de l'éthique de conviction. J'ai l'impression que, neuf fois sur dix, je me trouverai en présence d'outres pleines de vent qui n'ont pas réellement conscience des responsabilités qu'ils assument, mais qui se grisent au contraire de sensations romantiques. Cela ne m'intéresse guère humainement et ne m'émeut en aucune façon. Par contre je me sens bouleversé très profondément par l'attitude d'un homme mûr - qu'il soit jeune ou vieux qui se sent réellement et de toute son âme responsable des conséquences de ses actes et qui, pratiquant l'éthique de responsabilité, en vient à un certain moment à déclarer : " je ne puis faire autrement. Je m'arrête là ! » Une telle attitude est authentiquement [echt] humaine et elle est émouvante. Chacun de nous, si son âme n'est pas encore entièrement morte, peut se trouver un jour dans une situation pareille. On le voit maintenant : l'éthique de la conviction et l'éthique de la responsabilité ne sont pas contradictoires, mais elles se complètent l'une l'autre et constituent ensemble l'homme authentique, c'est-à-dire un homme qui peut prétendre à la « vocation politique».

Mes chers auditeurs, dans dix ans d'ici nous aurons peut-être l'occasion de reparler de cette question. À ce moment-là le crains fort, malheureusement, et pour de multiples raisons, que la réaction [184] 
n'ait depuis longtemps repris le dessus. Il est probable que très peu de choses parmi toutes celles que vous avez souhaitées et espérées, et je le confesse, que moi aussi j'avais espérées, se seront accomplies. Bien peu selon toute apparence - pour ne pas dire absolument rien. Je n'en serai pas brisé, mais je vous assure que cela pèse comme un fardeau sur celui qui en a conscience. Je voudrais bien voir dans dix ans ce que seront devenus ceux d'entre vous qui ont présentement le sentiment d'être de véritables «politiciens par conviction » et qui prennent part à la griserie de l'actuelle révolution, - je voudrais voir alors ce qu'ils seront intérieurement « devenus ». Ce serait vraiment trop beau si les choses pouvaient alors se passer comme dans Shakespeare, au sonnet 102 :

Notre amour était jeune, alors en son printemps, Quand pour le célébrer mes lais retentissaient ; Ainsi devant l'été Philomèle s'évertue Et interrompt son chant quand mûrissent les jours.

Mais les choses n'en sont pas là. Peu importe quels seront les groupes politiques qui triompheront : ce n'est pas la floraison de l'été qui nous attend, mais tout d'abord une nuit polaire, glaciale, sombre et rude. En effet, là où il n'y a rien, ce n'est pas seulement l'empereur qui a perdu ses droits, mais aussi le prolétaire. Et lorsque cette nuit se sera lentement dissipée, combien encore vivront, de tous ceux qui ont vécu l'actuel printemps, au visage si opulent? Que seront-ils tous devenus en leur for intérieur? Il ne leur restera qu'amertume ou grandiloquence, ou, bien encore la simple acceptation résignée du monde et de leur métier, ou bien enfin ils auront adopté une dernière solution qui n'est pas la moins fréquente : le renoncement mystique au monde, chez tous ceux qui en ont la grâce, à moins que - comme cela arrive malheureusement trop souvent - ils ne s'y soient contraints par mode. Dans [185] tous ces cas, je tirerai la conclusion suivante : ils n'étaient pas à la hauteur de leur tâche, ils n'étaient pas de taille à se mesurer avec le monde tel qu'il est et tel qu'il se présente ordinairement; en aucun cas ils ne possédaient ni objectivement ni positivement, au sens profond du terme, la vocation de la politique que pourtant ils croyaient avoir. Ils auraient mieux fait de cultiver modestement et 
simplement la fraternité d'homme à homme et pour le reste vaquer bonnement à leur travail quotidien.

La politique consiste en un effort tenace et énergique pour tarauder des planches de bois dur. Cet effort exige à la fois de la passion et du coup d'œil. Il est parfaitement exact de dire, et toute l'expérience historique le confirme, que l'on n'aurait jamais pu atteindre le possible si dans le monde on ne S'était pas toujours et sans cesse attaqué à l'impossible. Mais l'homme qui est capable de faire un pareil effort doit être un chef, et non pas seulement un chef, mais encore un héros, dans le sens le plus simple du mot. Et même ceux qui ne sont ni l'un ni l'autre sont obligés de s'armer de la force d'âme qui leur permettra de surmonter le naufrage de tous leurs espoirs. Mais il faut qu'ils s'en arment dès à présent, sinon ils ne seront même pas capables de venir à bout de ce qu'il est possible de faire aujourd'hui. Celui qui est convaincu. qu'il ne s'effondrera pas si le monde, jugé de son point de vue, est trop stupide ou trop mesquin pour mériter ce qu'il prétend lui offrir, et qui reste néanmoins capable de dire « quand même ! », celuilà seul a la « vocation » de la politique.

FIN 